\title{
Designing Security-enhanced Embedded Systems: Bridging Two Islands of Expertise
}

by

\author{
Maria Vasilevskaya
}

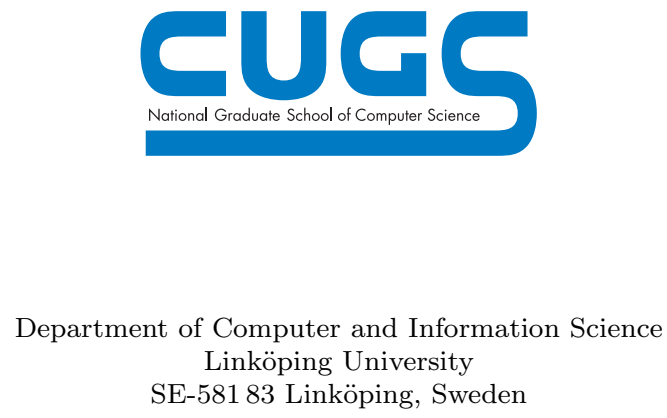

Linköping 2013 
This is a Swedish Licentiate's Thesis

Swedish postgraduate education leads to a Doctor's degree and/or a Licentiate's degree.

A Doctor's degree comprises 240 ECTS credits (4 year of full-time studies).

A Licentiate's degree comprises 120 ECTS credits.

Copyright (C) 2013 Maria Vasilevskaya

ISBN 978-91-7519-486-8

ISSN 0280-7971

Printed by LiU Tryck 2013

URL: http://urn.kb.se/resolve?urn=urn:nbn:se:liu:diva-98213 


\title{
Designing Security-enhanced Embedded Systems: Bridging Two Islands of Expertise
}

by

\author{
Maria Vasilevskaya \\ October 2013 \\ ISBN 978-91-7519-486-8 \\ Linköping Studies in Science and Technology \\ Licentiate Thesis No. 1624 \\ ISSN 0280-7971 \\ LiU-Tek-Lic-2013:58
}

\begin{abstract}
The increasing prevalence of embedded devices and a boost in sophisticated attacks against them make embedded system security an intricate and pressing issue. New approaches to support the development of security-enhanced systems need to be explored. We realise that efficient transfer of knowledge from security experts to embedded system engineers is vitally important, but hardly achievable in current practice. This thesis proposes a Security-Enhanced Embedded system Design (SEED) approach, which is a set of concepts, methods, and tools that together aim at addressing this challenge of bridging the gap between the two areas of expertise.
\end{abstract}

First, we introduce the concept of a Domain-Specific Security Model (DSSM) as a suitable abstraction to capture the knowledge of security experts in a way that this knowledge can be later reused by embedded system engineers. Each DSSM characterises common security issues of a specific application domain in a form of security properties, which are further linked to a range of solutions.

As a next step, we complement a DSSM with the concept of a Performance Evaluation Record (PER) to account for the resource-constrained nature of embedded systems. Each PER characterises the resource overhead created by a security solution, a provided level of security, and the evaluation technique applied.

Finally, we define a process that assists an embedded system engineer in selecting a relevant set of security solutions. The process couples together (i) the use of the security knowledge accumulated in DSSMs and PERs, (ii) the identification of security issues in a system design, and (iii) the analysis of resource constraints of a system and available security solutions. The approach is supported by a set of tools that automate its certain steps.

We use a case study from a smart metering domain to demonstrate how the SEED approach can be applied. We show that our approach adequately supports security experts in description of knowledge about security solutions in the form of formalised ontologies and embedded system engineers in integration of an appropriate set of security solutions based on that knowledge.

This work has been supported by the Swedish National Graduate School of Computer Science (CUGS) and the EU FP' SecFutur Project.

Department of Computer and Information Science

Linköping University

SE-581 83 Linköping, Sweden 



\section{Acknowledgement}

I would like to express my gratitude to my supervisor Simin Nadjm-Tehrani for the support that she provided to me over the recent years. Her tireless assistance and guidance have helped me to learn and progress with my research.

I gratefully acknowledge Linda Ariani Gunawan, Peter Herrmann, David Broman, and Kristian Sandahl with whom I had many fruitful discussions regarding my research and teaching. Special thanks go to David Broman for his unlimited support and inspiration as my mentor. Thanks to my secondary advisor Nahid Shahmehri for her feedback given at our meetings and when reading some parts of this thesis.

I wholeheartedly thank all current and former members of RTSLab for their friendship, support, and all the valuable comments during numerous RTSLab meetings. My appreciation extends also to members of other divisions of IDA with whom I happened to interact regarding my research and teaching.

I would like to thank all administrative personnel who make our working environment very pleasant. Special thanks go to Anne Moe, Eva Pelayo Danils, and Åsa Kärrman. It would have been much more difficult to work effectively without their professional support and patience.

Last but not least, I am thankful to my family for their support throughout these years. The encouragement and valuable assistance provided by Anatoly and Dmitry helped a lot in preparation of this thesis. I am sincerely thankful to my parents Elena and Viktor as well as to others for their care during these years.

Undoubtedly, there were many other people who contributed to my work with their support, advice or rewarding moments spent together. Unfortunately, it is not feasible to name everyone. Therefore, I anonymously thank all of you who did not find their name here but has contributed with their effort or time at any point of this journey. 



\section{Contents}

1 Introduction $\quad 1$

1.1 Motivation ................... . . 1

1.2 Problem Formulation . . . . . . . . . . . . . . . . . . . . . . . . 3

1.3 Contributions . . . . . . . . . . . . . 3

1.4 List of Publications . . . . . . . . . . . . . . . . . 4

1.5 Outline ....................... 5

2 Background and Case Study $\quad 7$

2.1 Embedded Systems Engineering . . . . . . . . . . . . 7

2.2 Modelware Zoo . . . . . . . . . . . . . . . . . . . . . 10

2.2.1 Main Concepts . . . . . . . . . . . . . 10

2.2 .2 UML . . . . . . . . . . . . . . . . . . . . . . . . . . . . 15

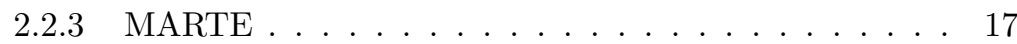

2.2 .4 SPACE ...................... 18

2.2 .5 Tools ....................... . . 21

2.3 Ontology Technologies . . . . . . . . . . . . . . 22

2.4 Case Study: Metering Infrastructure . . . . . . . . . . . . . 24

3 SEED: Bird's Eye View $\quad 27$

3.1 Introduction to SEED . . . . . . . . . . . . . . . . 27

3.2 The SEED Foundation . . . . . . . . . . . . . . . . . . . . . . . . 28

3.2.1 Creation of a System Model . . . . . . . . . . . . 28

3.2.2 Capturing the Domain-specific Security Knowledge . . 29

3.2.3 Development of a Security-enhanced Embedded System 30

4 Capturing of the Domain-specific Security Knowledge 33

4.1 Developed Concepts and Artefacts . . . . . . . . . . . . . 33

4.1.1 Domain-specific Security Model . . . . . . . . . . . 34

4.1.2 Performance Evaluation Record . . . . . . . . . . . . . 40

4.2 Capturing Security Knowledge . . . . . . . . . . . . . . . 47 
5 Application of the Domain-specific Security Knowledge 51

5.1 System Model . . . . . . . . . . . . . . . . . 52

5.1.1 Modelling a Functional Behaviour of a System . . . . 53

5.1.2 Modelling an Execution Platform . . . . . . . . . . 55

5.2 Association with DSSMs . . . . . . . . . . . . . 56

5.3 Asset Elicitation and Search for Security Properties . . . . . 57

5.3.1 Asset Elicitation on a Functional Model . . . . . . . . 57

5.3.2 Search for Security Properties . . . . . . . . . . 62

5.3.3 Asset Elicitation Utilising a Platform Model . . . . . . 63

5.4 Search for Concrete SBBs . . . . . . . . . . . . . 66

5.5 Compatibility-based Selection of Concrete SBBs . . . . . . . 68

5.5.1 Introduction into the Compatibility Analysis . . . . . 69

5.5.2 Ontologies for Compatibility Analysis . . . . . . . . 70

5.5.3 Model-based Compatibility Analysis . . . . . . . . . . 74

5.5.4 Scalability and Performance . . . . . . . . . . 77

5.6 Extended Form of the Process . . . . . . . . . . . . 79

6 Related Work $\quad \mathbf{8 1}$

6.1 Composing a System from Reusable Blocks . . . . . . . . . . 81

6.1.1 Component-based Development . . . . . . . . . 81

6.1.2 Aspect-oriented Development . . . . . . . . . . . 83

6.2 Security-enhanced System Design . . . . . . . . . . . . . 84

6.2.1 General Methods to Deal with Security . . . . . . . . 85

6.2.2 Methods for Embedded Systems . . . . . . . . . . . 87

6.2.3 Selection of Security Measures . . . . . . . . . . . . 88

6.2.4 Risk Analysis . . . . . . . . . . . . . . . . . . . 89

6.3 Marrying Ontologies and Models . . . . . . . . . . . . . 89

6.4 Performance Analysis at the Design Phase . . . . . . . . . . . 90

7 Conclusions and Future Work 93

7.1 Conclusions . . . . . . . . . . . . . . . . 93

7.2 Future Work . . . . . . . . . . . . . . 96 


\section{List of Figures}

2.1 Life cycle process models _ . . . . . . . . . . . . . . 9

2.2 Simplified design flow $[1] \ldots \ldots$. . . . . . . . . . . . 9

2.3 Models, meta-models, and meta-meta-models - the layered organisation . . . . . . . . . . . . . . 11

2.4 Model transformation: concepts and their relations . . . . . . 12

2.5 Relations between domains and languages, adapted from [2] . 14

2.6 Example of the stereotype definition and usage . . . . . . 16

2.7 Structure of the MARTE profile . . . . . . . . . . . 17

2.8 Structure of the MARTE analysis packages . . . . . . . . 18

2.9 SPACE model-based engineering method, adapted from [3] . 19

2.10 Model of a simple e-consultation application in SPACE $\quad \ldots \quad 20$

2.11 Smart metering infrastructure (an overview) . . . . . . . . 24

3.1 Generic process - the SEED foundation . . . . . . . . 28

3.2 Fragments of simplified design flows . . . . . . . . . . 31

4.1 DSSM concept and related artefacts . . . . . . . . . . 34

4.2 Core security ontology fragment . . . . . . . . . . . 35

4.3 UML representation of the core security ontology . . . . . . . 37

4.4 Fragment of the metering DSSM _ . . . . . . . . . . 38

4.5 Enriched security ontology . . . . . . . . . . . . . . . 39

4.6 PER concept and related artefacts . . . . . . . . . . . . . 41

4.7 Core evaluation ontology . . . . . . . . . . . . . . . 41

4.8 GEM UML profile to capture performance evaluation results 43

4.9 The refinement of the GEM profile for the security domain . 46

4.10 Fragment of the security evaluation record for the DES RBB 46

4.11 Enriched evaluation ontology . . . . . . . . . . . . 47

4.12 The process for creation of DSSMs . . . . . . . . . . 48

4.13 Registration of concrete SBBs tool (the user interface) . . . . 50

5.1 Application of the domain-specific security knowledge . . . . 51

5.2 Functional system model of the measurement transfer scenario 53

5.3 Detailed behaviour of the transfer handler block . . . . . . . 54

5.4 Platform model for a TSMC device . . . . . . . . . . . . . 55 
5.5 Association of the selected DSSM with the system elements (the user interface) . . . . . . . . . . . . . . 56

5.6 Rules of the asset identification technique . . . . . . . . . 59

5.7 Illustration of the rules . . . . . . . . . . . . . . . 60

5.8 Functions to traverse a functional system model . . . . . . . . 61

5.9 Asset analyser tool (the user interface) . . . . . . . . . . . 62

5.10 Asset elicitation technique utilising a platform model . . . . . 63

5.11 Adapted model protecting the transfer of measurement data . 68

5.12 Concrete SBB searcher tool (the user interface) . . . . . . . 69

5.13 Ontologies for compatibility analysis . . . . . . . . . 71

5.14 Classification levels for the developed ontologies (excerpts) . . 72

5.15 Model-based compatibility analysis tools (the user interfaces) 77

5.16 Extended form of the proposed process . . . . . . . . . . 79 


\section{List of Tables}

4.1 Correspondence between our GEM and (MARTE) GQAM . . 45

5.1 Results of eliciting assets from the functional model . . . . . 61

5.2 Association of the assets with the platform components . . . 65

5.3 Threats and violated security goals . . . . . . . . . . 65

5.4 Scalability and performance estimations . . . . . . . . . 78 



\section{Chapter 1}

\section{Introduction}

The ubiquitous presence of networked embedded devices is no longer questioned. Large computing infrastructures that bring automation in our daily lives exist due to support of such devices interconnected through networks. Being a part of such infrastructures, embedded devices carry and process sensitive information. Thus, both their exposure to open networks and their critical role in storing, processing, and transmission of information makes embedded devices a target of sophisticated attacks. The interest of attackers is stimulated by the fact that modern embedded systems are often easily accessible (e.g. deployed in a hostile environment) and the consequences of compromising such devices can be very large. If an attacker hacks a device of one type, the attack can be quickly replicated to all other devices of the same type possibly in thousands or millions. If attackers take control over one of the devices of a large network, they can gain access to other devices of the network. For example, a computer scientist at Colombia University, Ang Cui, has developed a technique that allows taking complete control of a Cisco IP phone, that in turn allows affecting other parts of a connected system (other phones in a network, computer, printers, etc.) [4]. These facts impose high requirements on security standards for embedded systems that are often neglected. To emphasise the point, McClure estimates [5] that there are already 10 billion embedded devices in operation that were designed without much thought about security.

Thus, it goes without saying that security issues should be considered during embedded system development since insufficient security can create a substantial risk for society and significant lost of profits for embedded system producers, owners, and end users.

\subsection{Motivation}

Although security is an essential aspect of networked embedded systems, it is still approached as an add-on late in the development process. This 
can hardly be effective due to the complexity of embedded systems, their resource-constrained nature, and non-functional requirements. For instance, Ravi et al. [6] discuss the main consequences of incorporating security solutions into embedded systems at the late development phases. Resources planned during the initial development phase do not account for security functions. These insufficient resource requirements dramatically limit the number of security solutions available for a system engineer or even put this number to zero. The authors identify a set of bottlenecks that system designers consequently have to deal with. These include, but not limited to, the energy consumption overheads (the battery gap) and the computational demands (the processing gap). Ravi et al. argue for a shift to an appropriate design methodology to address these challenges.

There is a number of factors that make attacks on embedded systems successful. An unthoughtful system design is one of the sources of potential breaches. Vulnerabilities introduced during the implementation phase are another one. Human factors such as an intentional and unintentional misuse of system components are major problems during the usage phase. While all factors are significant, this thesis focuses on resolving security issues at the design phase of the system development. The underlying reason for such a focus is that the consequences of an unthoughtful design influence all later phases of the system life cycle. At the same time, integration of security mechanisms already at the design phase allows early exploration of performance, power consumption, cost and other trade-offs. This motivates adopting the principles of model-based engineering [7] as a vehicle to bring security consideration to a design phase.

A mere focus on the design phase is not enough to efficiently tackle security issues. The challenge is amplified by the diversity and complexity of both security solutions and embedded systems as such. Embedded systems design requires in-depth understanding of an application domain, usage scenarios, and deployment environment. Security threats, in turn, vary from application to application and are more or less prevalent in an application domain. Due to these concerns, Kocher et al. [8] stress the need of system engineers (who are not necessarily experts in security) to understand both required level of security assurance and the overhead caused by injecting security solutions into a system design.

Security mechanisms should be developed and thoroughly studied by security experts, whereas the resulting knowledge should be available for embedded system engineers. Last but not the least, a security solution for embedded systems should be specific for a particular application domain in order to provide the required efficiency at the cost of acceptable performance. This brings us to two additional principles that we propose in this thesis to improve practices of security-enhanced embedded system development, namely the separation of roles (i.e. an embedded system engineer and security expert) and domain specialisation (i.e. application-driven security) principles. 


\subsection{Problem Formulation}

The objective of this thesis is to provide concepts and tools for addressing security issues of embedded systems already at the design phase. We aim to reach this goal by defining an approach which targets two categories of professionals. With the help of the developed approach, security experts should have an opportunity to describe developed security solutions in a reusable manner. This, in turn, should enable embedded system engineers to select a suitable set of security solutions based on the analysis of both system's security needs and system resource constraints. The approach should explore the following principles:

- Model-orientation: which allows dealing with security concerns already at the early development phase.

- Domain specialisation: which increases the quality and efficiency of eventual solutions by narrowing down the focus to a specific domain.

- Separation of responsibilities and concerns: which promotes separation of security expert and embedded system engineer roles.

Realising the basic principles stated above, we contribute to tackling the challenge of adequate support for a security expert and an embedded system engineer by answering the following questions:

- What abstractions and concepts are suitable for a security expert to assist in creating a useful description of a security mechanism?

- What technologies and processes can be employed to assist a security expert in capturing this knowledge?

- What are methods and tools that should equip an embedded system engineer to enable the use of the provided security knowledge to efficiently select a set of security solutions for their integration into a system design?

\subsection{Contributions}

The main contribution of this work is the definition of a Security-Enhanced Embedded system Design (SEED) approach. More specifically, our contributions consists of:

- a proposal of two concepts to represent the domain-specific security knowledge that encapsulates information about reusable security solutions, namely Domain-Specific Security Model (DSSM) and Performance Evaluation Record (PER),

- a process for a security expert to capture the domain-specific security knowledge, 
- a process for an embedded system engineer to reuse the captured knowledge at the system design phase,

- development of a MagicDraw [9] plug-in to support the processes described above.

The MagicDraw plug-in combines both modelling and ontology technologies to implement the DSSM and PER concepts.

The process defined for an embedded system engineer, in turn, rests on two other contributions of this thesis:

- a method called asset elicitation technique that analyses a system design to elicit a system's security needs;

- a method called model-based compatibility analysis that matches resource constraints of an embedded system under development and alternative security solutions.

\subsection{List of Publications}

The work presented in this thesis is based on the following publications:

- S. Nadjm-Tehrani and M. Vasilevskaya, Towards a Security Domain Model for Embedded Systems, in The 13th IEEE International Symposium on High Assurance Systems Engineering (HASE), poster session, IEEE, November 2011

- M. Vasilevskaya, L. A. Gunawan, S. Nadjm-Tehrani, and P. Herrmann, Security Asset Elicitation for Collaborative Models, in Model-Driven Security Workshop (MDSec) in conjunction with MoDELS, ACM, Innsbruck, Austria, Octorber 2012

- M. Vasilevskaya, L. A. Gunawan, S. Nadjm-Tehrani, and P. Herrmann, Integrating security mechanisms into embedded systems by domainspecific modelling, Journal of Security and Communication Networks, Wiley, June 2013.

- M. Vasilevskaya and S. Nadjm-Tehrani. Support for Cross-domain Composition of Embedded Systems Using MARTE Models. Submitted for review.

Some content of this thesis have been published as parts of deliverables of the EU FP7 SecFutur project [10]:

- Deliverable 3.1: Abstract model for embedded systems. Edited by Simin Nadjm-Tehrani and Maria Vasilevskaya, 2011.

- Deliverable 4.1: SecFutur Development Process V1 and Modelling Framework. Edited by Antonio Maña, Jose F.Ruiz, and Rajesh Harjani, 2011. 
- Deliverable 4.2: SecFutur development process V2 and code development and tool-suite V1. Edited by Jose F. Ruiz, Antonio Maña, and Marcos Arjona, 2012.

- Deliverable 4.3: SecFutur code development and tool-suite V2. Edited by Maria Vasilevskaya and Simin Nadjm-Tehrani, 2013

The following papers were authored or co-authored during the period of the presented work. The papers are not included in this thesis.

- B. Yatsalo, S. Gritsyuk, O. A. Mirzeabasov, and M. Vasilevskaya, Uncertainty Treatment within Multicriteria Decision Analysis with the Use of Acceptability Concept, Journal of Large-scale Systems Control, RAS, 2011.

- M. Vasilevskaya, D. Broman, and K. Sandahl, An Assessment Model for Large Project Courses, ACM Technical Symposium on Computer Science Education, SIGCSE, 2014.

\subsection{Outline}

The rest of this thesis is organised as follows. We provide the necessary background to our work in Chapter 2. Chapter 3 explains the idea and structure of SEED in general terms that are detailed in the following chapters. In particular, Chapter 4 defines the process for capturing of the domain-specific security knowledge. Chapter 5 explains methods and tools to use the captured knowledge for integration of protection mechanisms into an embedded system design. Thereafter, we provide a summary of some related work in Chapter 6. Finally, Chapter 7 concludes this thesis and gives some pointers for future work. 



\section{Chapter 2}

\section{Background and Case Study}

This chapter provides the necessary background needed in the context of this work. Section 2.1 gives an overview of the basic process models for embedded system engineering. Then, the basic concepts, tools, and methods of model-based engineering are introduced in Section 2.2 followed by a brief introduction to ontology technologies given in Section 2.3. We conclude this chapter by presenting a case study from the smart metering domain described in Section 2.4 that is used as a running example throughout this thesis to illustrate the introduced concepts, methods, and processes.

\subsection{Embedded Systems Engineering}

Development of embedded systems is a complex task. Therefore, a set of process models exist that support engineers in tackling this complexity. In a broad sense, a process defines a set of activities, their input/output artefacts, roles with responsibilities, tools, time frames, and costs. In our work, we are mainly concerned about activities and input/output artefacts.

At the level of main activities, life cycle models (i.e. process models) for development of embedded systems are very similar to life cycle models proposed for general software engineering. In particular, there are five basic steps that span across the whole life cycle of a system: requirements definition, system specification, functional design, architectural design, and prototyping/implementation [11]. The first step intends to capture the customer's wish in terms of what a system shall do. The following step, i.e. system specification, refines the customer description in a more concise and precise form. The next two steps go deeper and turn specifications into a set of functional blocks that are later mapped into architectural elements. These elements are combinations of hardware and software resources. Fi- 
nally, a system is implemented that results in a prototype. The extended life cycle models instrument these basic five steps with extra activities, such as testing, validation, verification, and maintenance. In the following, we outline three widely known life cycle models, namely waterfall, spiral, and $\mathrm{V}$ models.

- The waterfall [12] model depicted in Figure 2.1(a) represents a development cycle as a sequence of the steps above. According to this model, an engineer should proceed to the next step when the current phase is completed. Additionally, there is a feedback loop (depicted by the backward arrows) to the previous phase that ensures conformance of artefacts created on the current phase to the artefacts produced on the previous step. The presence of this feedback loop differs the waterfall model from a simple sequential process.

- The $V$ model [13] depicted in Figure 2.1(b) is similar to the waterfall model, but it emphasises the verification and validation activities. A system development follows the top-down approach (the left-hand side), while the verification and validation activities go from the bottom to the up (the right-hand side). Thus, the implemented system is verified against each produced artefact, namely implementation, architectural design, functional design, specification, and requirements. Unit and integration testing verifies a system against the artefacts created at the prototyping/implementation phase, e.g. program design.

- The spiral model [14] depicted in Figure 2.1(c) promotes an iterative style for development of a system. Thus, the main difference of the spiral model and the above mentioned models is that it emphasises iterative emergence of several versions of the same system. First, a very restrictive version of a system is developed to understand if the requirements are correctly and adequately formulated. Then, the system evolves into more complex and complete versions, e.g. prototype, initial design, and enhanced design. The corresponding artefacts, e.g. requirement specifications, functional and architectural designs, implementation, also evolve. The radius of the spiral can reflect the amount of time spent on each cycle.

Different variations, modifications, and combinations of the presented process models exist. For example, Douglass [15] proposes a so called harmony development process (see Figure 2.1(d)) where the $\mathrm{V}$ and spiral models are combined.

The embedded systems community also poses a process for embedded system development that differentiates two distinct levels. They are system and lower levels. In this approach, verification, validation, testing, estimation, and analysis steps are tightly woven into a process. The system level concerns defining a system model and selecting a suitable architecture. 


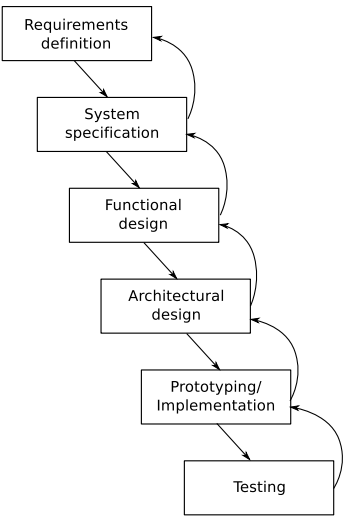

(a) Waterfall model

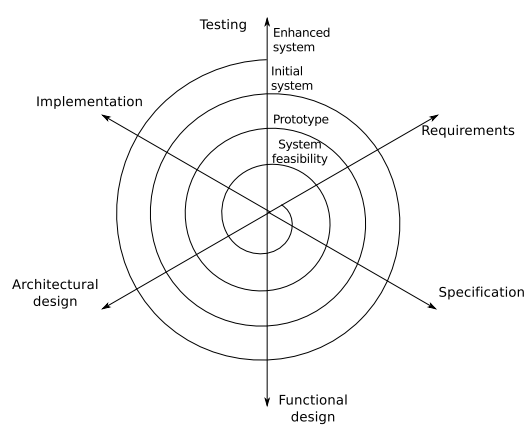

(c) Spiral model

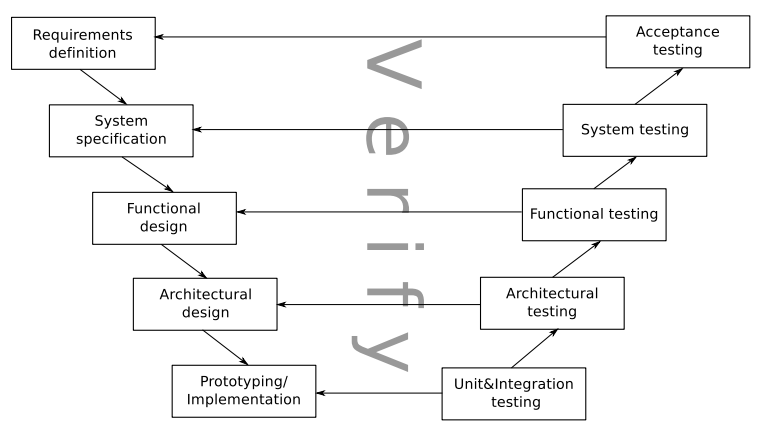

(b) $\mathrm{V}$ model

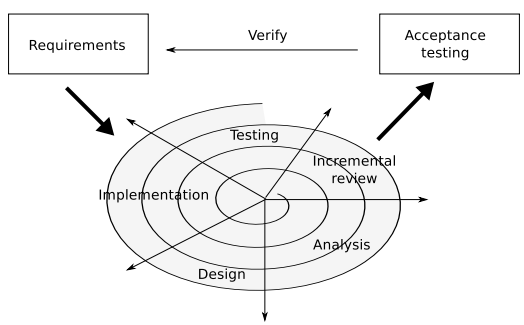

(d) Harmony model

Figure 2.1: Life cycle process models

These artefacts are further evolved into different parts of code (RTOS and application code) and elements of hardware at the lower level.

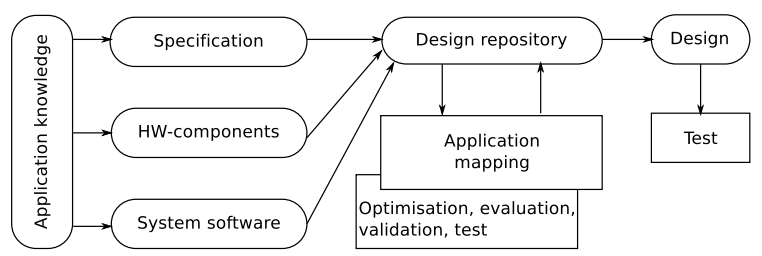

Figure 2.2: Simplified design flow [1]

The last model for the life cycle development that we visualise in this section is the simplified design flow presented by Marwedel [1]. Figure 2.2 depicts this process. This model does not radically differ from the models presented above. The design starts from some application knowledge 
that is transformed into specification, and hardware/software components. However, Marwedel explicitly brings the design repository into the process. According to Marwedel, this repository serves to keep track of design models evolution. However, we envisage wider use of this component, namely as a point to extend and refine the initial design. This idea is further exploited and evolved in our work.

\subsection{Modelware Zoo}

In this section, we briefly introduce the reader to the area of model-based engineering. First, we cover main concepts of the modelling theory. Thereafter, we describe two modelling languages used in our work, namely UML and MARTE, and an employed system modelling approach called SPACE together with its modelling language. We conclude this section outlining tools that support principles of model-based engineering.

\subsubsection{Main Concepts}

We begin with introducing terms of models, meta-models, transformation, and basics of the language engineering, followed by brief discussions on topics such as domain-specific compared to general modelling, and model-based compared to model-driven engineering.

\section{Models and Meta-models}

In general, models allow to raise the abstraction level to deal with growing complexity of artefacts (e.g. embedded system design) [16]. Abstraction improves understanding of complex artefacts and allows their efficient analysis through hiding some irrelevant information. In other words, a model represents a real system highlighting its properties of interest.

Any model conforms to some meta-model that defines its properties. Thus, a meta-model defines a modelling language used to create a model of a certain type for a system. Depending on the type of properties that a model should describe an employed meta-model will change. Consequently, a metamodel conforms to some language used to define properties of this metamodel, i.e. a meta-meta-model. In theory, an infinite hierarchy of modelmeta-model relations can be specified. However, in practice, meta-metamodel is abstract and general enough to define itself wrapping the layered organisation of modelware (see the left side of Figure 2.3). Such organisation is sometimes referred as the four-layered architecture (M0-M3) [17] or $3+1$ organisation [18].

The right side of Figure 2.3 depicts a classical example that demonstrates an instantiation of the layered organisation introduced above. A real-world object (car) is shown at level M0. A model of the car is shown at level M1. This model describes a car as a Car class with one "colour" attribute. The 
meta-model located at M2 explains how to understand this model, namely what elements are classes and what elements are attributes. Finally, level M3 defines concepts used at level M2. Thus, both Attribute and Class are represented as classes at $\mathrm{M} 3$.

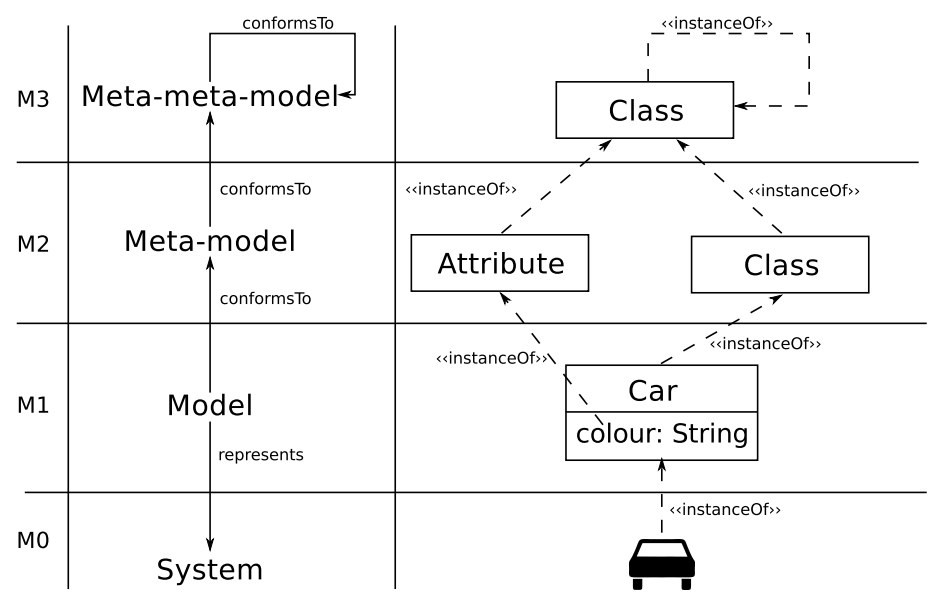

Figure 2.3: Models, meta-models, and meta-meta-models - the layered organisation

The Object Management Group (OMG) [19] implements the M3 level as the Meta-Object Facilities (MOF) standard [20]. MOF is used to define the Unified Modelling Language (UML) [17] located at the M2 level.

\section{Transformation}

Model transformation is a technique that allows defining a mapping between different models, i.e. source and target models, that are different representations of the same system. Figure 2.4 depicts a classical scheme that explains concepts of model transformation and their relations. Any model transformation is applied to source and target models, but the actual transformation is defined at the meta-model level, i.e. a model transformation definition refers to elements of the meta-models of the source and target models. Thus, model transformation receives input and output models that conform to their respective source and target meta-models. At the same time, a model transformation definition is a model by itself that conforms to some meta-model, i.e. to a transformation language $[18,7]$.

Basically, transformation languages can be classified as declarative, imperative, and hybrid. Declarative languages require an engineer to specify relations between source and target meta-models, e.g. in terms of functions. In contrast, one needs to specify such details as execution order (sequence of steps) when imperative languages are used. A hybrid type of languages is an intermediate category that mixes constructs and principles from both 


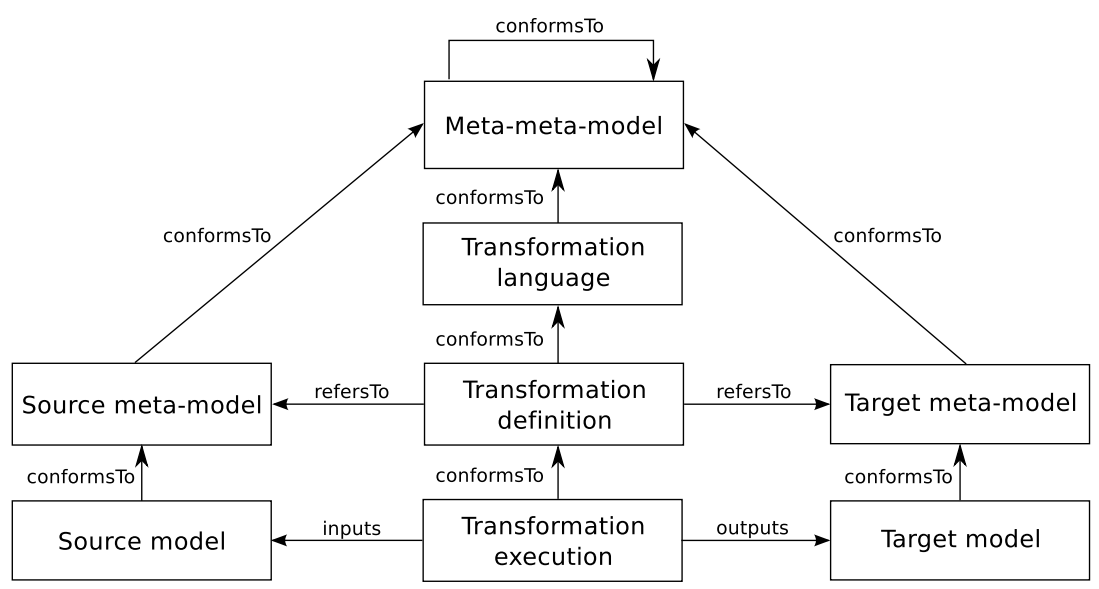

Figure 2.4: Model transformation: concepts and their relations

declarative and imperative languages.

Depending on the nature of target and source meta-models, transformation languages can be classified as model-to-model (M2M) and model-to-text (M2T) transformations [21]. Naturally, the former type of transformations input a model conforming to a certain meta-model (e.g. UML) and produce another model that conforms to a different meta-model (e.g. EntityRelation Diagram), while the latter type of transformation results in some textual representation (e.g. Java code). Recently, a third type of transformation called text-to-model (T2M) has been introduced. Additionally, one can classify a transformation as endogenous or exogenous. A transformation is considered to be endogenous if source and target models conform to the same meta-model. In contrast, an exogenous transformation is used when source and target models conform to different meta-models.

Model transformation is a powerful concept that is used to automate different tasks of model-based engineering [22]. For example, code generation is a special type of model transformation where the target model is code. Model composition, model refactoring, verification, and reverse engineering are other examples of scenarios where model transformation can be applied.

\section{Abstract Syntax, Concrete Syntax, and Semantics}

To enable sophisticated operations with models (e.g. transformation), they must have a well-defined structure. Therefore, techniques for systematic definition of meta-models should be used. The research area that concerns proper definition of complex modelling languages is sometimes referred to as modelling language engineering [23].

The main elements that define a language are its syntax (i.e. a language's notation) and semantics (i.e. a language's meaning). There are two types 
of syntax that serve for different purposes, namely an abstract syntax and a concrete syntax [24]. An abstract syntax defines all valid models of modelling languages. For example, an abstract syntax defines what are concepts of a modelling language (e.g. classes and their attributes) and what are their valid relations (e.g. associations). Meta-modelling (see Figure 2.3) is a technique for defining an abstract syntax. A concrete syntax defines how an abstract syntax appears for an engineer (i.e. for its users). Thus, a concrete syntax deals with representation of a modelling language. A concrete syntax can be represented in textual or visual (e.g. boxes and arrows) notations.

Semantics defines the meaning of a language notation (i.e. syntax). In general, there are two steps to define semantics for a language. First, a semantic domain should be defined that provides a meaning for each expression. This meaning must be an element of another well-understood domain, e.g. real numbers. Afterwards, a semantic mapping should be created to bound elements of an abstract syntax to a defined semantic domain.

\section{Domain-specific vs. General-purpose Modelling}

Model-based engineering methods distinguish two big categories of modelling languages, namely Domain-Specific Modelling Languages (DSMLs) and General-Purpose Modelling Languages (GPMLs). DSMLs are languages that are designed for a certain domain [25]. Such languages are usually designed by a group of experts to be used in a specific context or company to facilitate a particular task (e.g. the task of describing things in that domain). In other words, a DSML allows the user to specify a solution using terms of a problem domain that are built in this DSML. Besides, DSMLs are intended to support a better reuse of functionality recurring in a set of modelling tasks. Thus, a DSML is optimised for a certain class of problems within a domain. In contrast, a GPML does not target a specific domain, but rather is intended to be applied in any domain.

Since expressiveness of DSMLs is bound to a particular domain, they can be used only for a predefined set of problems. In contrast, GPMLs are advertised to be suitable for a wide range of modelling tasks. However, DSMLs bring higher productivity and conciseness in modelling since an engineer operates with a limited set of concepts that are familiar and intuitive for a considered domain.

There are a lot of discussions on the topic of "DSML vs. GPML". Both classes of languages are suitable for different purposes and scenarios. Therefore, it is rational to be aware of their advantages and disadvantages through their systematic comparison. Boundaries between domain-specialisation are not obvious: any language is more or less domain-specific [2]. In this section, we outline some characteristics that are typical for a pure DSML and GPML.

A pure DSML can be characterised by the following set of peculiarities: it is designed for a small and well-defined domain; it has a relatively small size with a limited set of user-defined abstractions; its development takes 
months to years; it is designed by a few domain experts; its user community is a small and accessible group. In contrast, a real GPML is designed for a large and complex domain; it has a large language size with a sophisticated set of general abstractions; its development usually spans over years and decades; it is designed by gurus and large communities.

To conclude our discussions about DSMLs and GPMLs, Figure 2.5 illustrates views on relations between domains and languages presented by Voelter [2]. Figure 2.5(a) shows the relations between domains as a hierarchical structure where a domain of a pure GPML is the lowest level. An example of a languages order based on their domain-specificity is depicted in Figure 2.5(b). We believe that these figures give a good intuition on boundaries between domain-specific and general-purpose modelling languages.

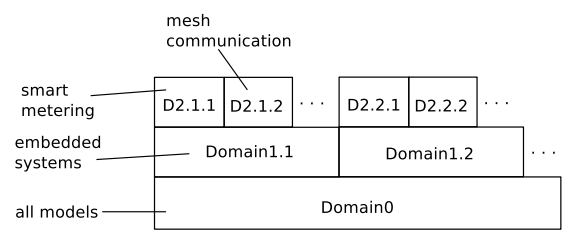

(a) Domain hierarchy

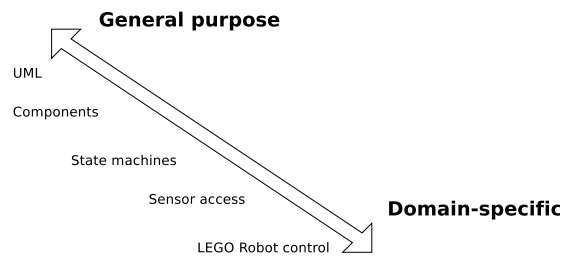

(b) Language order

Figure 2.5: Relations between domains and languages, adapted from [2]

\section{Model-based vs. Model-driven Engineering}

A set of development paradigms that rely on models as a key artefact have recently emerged. They are Model-Based Engineering (MBE), Model-Driven Development (MDD), Model-Driven Engineering (MDE), and Model-Driven Architecture (MDA). However, these techniques can be distinguished based on the role of models in the proposed processes.

To begin with, we briefly explain the difference between "model-driven" and "model-based" prefixes. Intuitively, the latter is a softer version of the former: the former prefix says that models drive the process, while in the latter case models play an important role, but are not key artefacts. In case of "model-driven", it is often expected that a model is used to generate the final implementation. In contrast, for the "model-based" techniques, a model can be used for various kinds of analysis and even test generation, but the actual implementation can be done by developers. Thus, MDE can be considered as a subset of MBE [7]. Similarly, MDD is a subset of MDE, since the letter "D" stands for "Development" that is one type of activity in system engineering. Finally, MDA is a realisation of MDD proposed by Open Management Group (OMG) [19] that is inherently based on OMG standards. 


\subsubsection{UML}

Unified Modelling Language (UML) is a widely accepted general purpose modelling language. Modelling concepts defined by UML are organised in different types of diagrams. The current version of UML (v2.4.1) [17] differentiates 14 types of diagrams. These diagrams are classified in two categories: those that are intended to model structural and behaviour parts of a system. Each type of diagram uses different modelling concepts that together allow describing diverse aspects of a system.

There are seven types of structural diagrams. A class diagram shows system's classes, their attributes, their operations, and the relations among classes. A component diagram shows the components of a system and their relations. A composite structure diagram shows the internal structure of a class and the interaction (collaboration) that this structure enables. A deployment diagram can be used to show the hardware/software parts of a system and artefacts deployed on this execution environment. An object diagram shows instantiation of the system classes. A package diagram shows the logical organisation of a system as a set of packages and their dependencies. Finally, a profile diagram encapsulates custom domain-specific extensions of the standard UML constructs (see below).

Behaviour of a system can be described using seven types of diagrams. An activity diagram can be used to show a workflow (both control and data) of a system. A state machine diagram shows the system's states and their transitions. A use case diagram is used to give a high-level description of a system in terms of actors, their goals, and dependencies between actors and goals. Communication and sequence diagrams are used to describe the interaction and communication between objects as sequences of messages using different syntaxes. The last two types of diagrams are interaction overview and timing diagrams that enable creation of an overview of a system and specifying some timing constraints of operations respectively.

This rich set of diagrams allows capturing different aspects of a system. All together, they have a complex and diverse syntax, but rather weak semantics. As a result, UML diagrams are used for modelling tasks in many different domains, but these models are not comparable due to the absence of a commonly agreed semantic domain. Therefore, usually a small subset of UML (i.e. some syntactical constructs such as a subset of UML class diagrams) is used and is further supported by a user-defined semantics bound to a considered domain.

In addition, the UML standard defines extensibility mechanisms that can be used to add domain-specificity to UML. They are profiles, stereotypes and tag values. A profile is a special type of package that contains stereotypes. A visual representation of a profile is referred to as a profile diagram. A stereotype allows adding a set of specific properties (suitable for a particular domain) into existing UML concepts (e.g. class, activity, component). Thus, a stereotype can be considered as a mechanism to refine existing UML concepts with required non-standard semantics. Each 
stereotype extends (refines) some UML base meta-class (e.g. class, property, named element). Therefore, a stereotype can be used to annotate only those concepts that extend the same meta-class. A stereotype can introduce additional domain-specific properties. These properties are defined through so called tag values. Figure 2.6 depicts a small example of a stereotype definition and its usage. Figure 2.6(a) shows a stereotype called Car that has two attributes, namely colour and brand. Thereafter, we have applied the stereotype Car to specialise the class BondCar (see Figure 2.6(b)). Hence, BondCar has all the properties declared for the Car stereotype. The colour and brand properties can be assigned to some values using tag values. For our example in Figure 2.6(b), they are black and Aston Martin respectively.

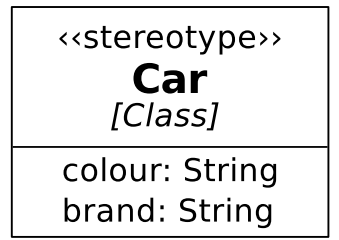

(a) Definition of a stereotype

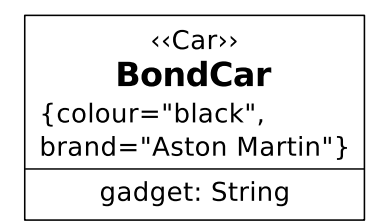

(b) Usage of a stereotype and tag

Figure 2.6: Example of the stereotype definition and usage

Note, that a stereotype should not be confused with the inheritance relation. Annotation of entities with a certain stereotype does not bring the classical child-parent dependency. In our example, if we remove the stereotype Car from the model, the class BondCar will still exist but without the colour and brand properties that belong to the stereotype Car. In contrast, a child can not exist without a parent when the inheritance relation is established.

One can distinguish two main approaches to defining a UML profile [26]. The first approach starts directly by defining a set of stereotypes that extend the UML meta-model. The second approach introduces a more systematic two-stage process.

According to the latter approach, an engineer first needs to create a conceptual model for a domain. A conceptual model describes all (relevant) concepts of a selected domain and their relations. In the second stage, the actual set of stereotypes together with their attributes and constraints is derived from a conceptual model. This process is sometimes referred to as mapping [27]. Lagarde et al. [26] suggest to automate this step to avoid errors and to enable relevant verifications to ensure consistency of the resulting profile with its conceptual model. In the context of the modelling language engineering explained in Section 2.2, the mentioned conceptual model can be referred to as an abstract syntax and a profile as a concrete syntax. 


\subsubsection{MARTE}

MARTE [28] is a standardised UML profile designed for Modelling and Analysis of Real-Time Embedded systems. It contains a rich set of concepts to support design and analysis of embedded systems. The structure of this profile is outlined in Figure 2.7. The MARTE foundations package provides a set of concepts required to model non-functional properties (the NFP package), time properties (the Time package), generic resources of an execution platform (the GRM package), and resource allocation (the Alloc package). These foundations serve as basics for the MARTE design and analysis models.

The design package (or model) contains sub-packages to describe the hardware and software resources, namely Hardware Resource Modeling (HRM) and Software Resource Modeling (SRM). Additionally, the design modelling packages contains concepts to model a component structure and application features. These concepts are encapsulated into the Generic Component Model package (GCM) and High-Level Application Modeling (HLAM) packages. The MARTE analysis package provides facilities to model the context required to perform analysis of real-time and performance characteristics of embedded systems. In particular, the Generic Quantitative Analysis Modeling (GQAM) package defines a set of general terms while its extensions refine them to support schedulability (SAM) and performance analysis (PAM).

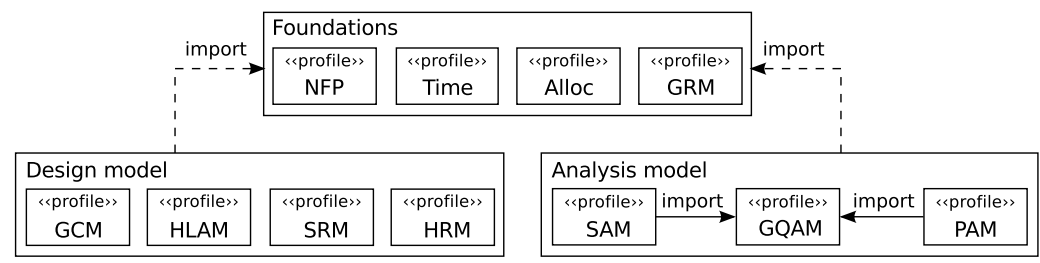

Figure 2.7: Structure of the MARTE profile

Figure 2.8 shows the basic elements of the GQAM package. Its central concept is the Analysis Context. This concept aggregates all relevant information needed to describe the constitutents of any type of analysis. In particular, the analysis context concept relates resource platform and workload behaviour elements. A workload behaviour defines a set of system operations that are triggered over time by a set of workload events. A resource platform is a container for resources, i.e. hardware/software execution platform, that are used by the system operations mentioned above.

Several experience reports on applying the MARTE profile to industrial cases exist. In general, we can distinguish the following strategies for MARTE exploitation:

- Strategy 1: The whole design process is maintained using MARTE. 


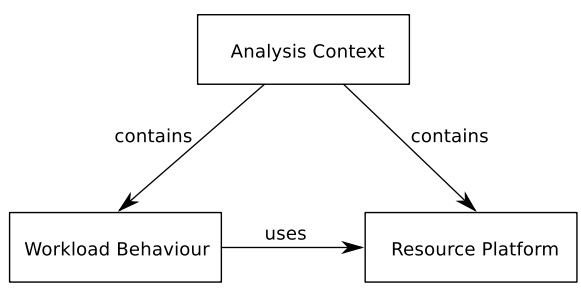

Figure 2.8: Structure of the MARTE analysis packages

- Strategy 2: A part of MARTE is reused. The rest of modelling is maintained extending MARTE with new concepts or using another profile together with MARTE.

- Strategy 3: A currently used DSML is adapted and mapped onto MARTE constructs. Additionally, some new concepts existing in a selected DSML are created to extend MARTE.

André et al. [29] and Zimmermann et al. [30] explore some MARTE packages to model System-on-Chip and Network-on-Chip systems respectively, employing strategy 2. In particular, they use time and hardware resource modelling capabilities of MARTE. Some concepts added into MARTE concepts are Bridge (an extension of the MARTE HwBridge stereotype), Port (an extension of the HwEndPoint stereotype), and CPU (an extension of the HwProcessor stereotype). Following the same strategy, Iqbal et al. [31] use concepts from the NFP and GRM packages together with a set of concepts from another profile to encompass the task of model-based robustness testing. Bernardi et al. [32] and Cancila et al. [33] define two profiles for dependability (DAM) and safety (SOPHIA) analyses extending the MARTE analysis modelling packages.

Espinoza et al. [34] present results of the ADAMS project [35] where standards from the automative domain, namely AUTOSAR and EAST-ADL2, are aligned with MARTE. This use of MARTE corresponds to strategy 3 of our classification. Demathieu et al. [36] present their experience on applying MARTE for an academic case study where a robot system is modelled using the SRM and HRM MARTE packages. This work is an example where strategy 1 within our classification is employed.

\subsubsection{SPACE}

We proceed to describe a method that employs a modelling language that is based on a subset of the UML standard enriching it with the formal semantics.

SPACE is a model-based engineering method [3] supported by the Arctis tool-set [37]. When this method is used, applications are composed of 
building blocks that can specify local behaviour as well as the interaction between several distributed entities. This specification style enables a rapid application development since, on average, more than $70 \%$ of a system specification comes from reusable building blocks provided in domain-specific libraries [38]. In turn, this strategy helps to reduce the expertise required in developing cross-domain applications. An additional benefit is the formal semantics of the specification defined by Kraemer and Herrmann [39], which makes it possible to verify system properties, e.g. that the building blocks are correctly integrated into activities [37].

Figure 2.9 gives an overview of the SPACE method [39]. An engineer starts studying a library of reusable building blocks. In case a needed building block does not exist in the library, an engineer can start creating a new one and add it into the library for its further reuse. Each building block can cover the behaviour of a single component as well as collaborative behaviour among several components. Building blocks can be domain-specific or quite general that can be integrated into several systems. Each building block is described as a combination of UML collaborations (an element of a UML composite structure diagram), activities (an element of a UML activity diagram), and so-called external state machines (ESMs) that specify externally visible behaviour of building blocks. Several building blocks are composed into a system with desired services. At this stage, analysis of a composed system (e.g. verification of functional or safety properties) can be performed due to the defined transformation of collaborative models into a temporal logic formula that serves as an input to the TLA (Temporal Logic of Actions) model checker [40]. Thereafter, the resulted system design is automatically transformed into state machines, that can be further used to generate implementation code via relevant transformations.

Our contribution enhances the step composition and analysis from Figure 2.9 when it comes to decide on a set of security measures expressed as reusable building blocks. In particular, we elaborate a method to select a set of security building blocks that are suitable for a system under development according to identified security needs.

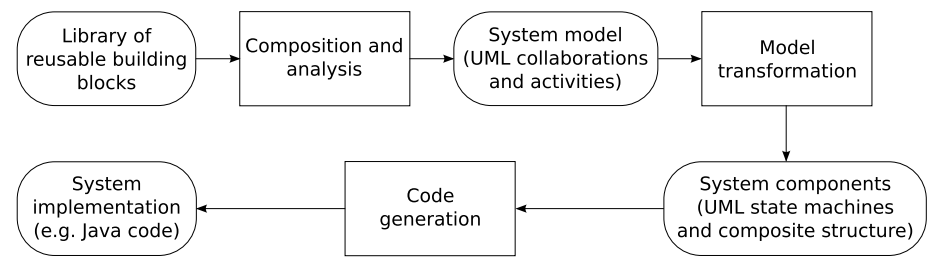

Figure 2.9: SPACE model-based engineering method, adapted from [3]

In the following, we explain elements of the modelling language used by SPACE, namely local blocks, collaborative blocks, and external state machines. We use a small example of a simple e-consultation application 
depicted in Figure 2.10 to demonstrate the introduced elements. In this scenario, a customer sends a question to a consultant. The consultant processes the question and sends a reply to the customer. The system structure is specified by a UML collaboration as shown in Figure 2.10(a). On this diagram, the collaboration roles depicted as rectangles represent two components of the system, namely a Customer and a Consultant. These two components are bound to the client and server roles respectively. The collaboration use, namely the chart:Simple Chart block, that is depicted as an ellipse encapsulates a logic of the component interaction.

Figure 2.10(b) shows the behaviour view of the system that is modelled as a UML activity with a slightly modified syntax. The e-consultation scenario is built of two partitions, i.e. the client and the server, that model the corresponding entities, i.e. a customer and a consultant. These partitions include three building blocks (instantiated as call behaviour actions), namely cm:Customer, cnt:Consultant, and chart:Simple Chart. The former two blocks model the local behaviour and are denoted local blocks, while the latter block models interaction between entities and called collaborative block. Each of these blocks is associated with another UML activity that details their behaviour (not shown in Figure 2.10).

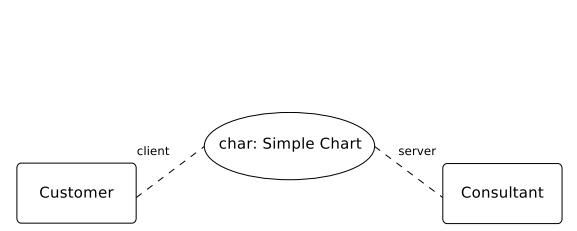

(a) UML Collaboration

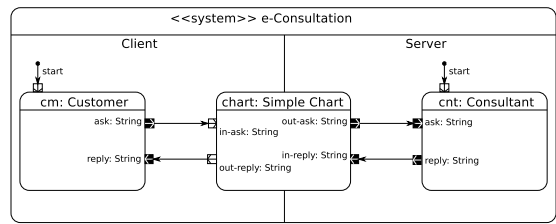

(b) UML Activity

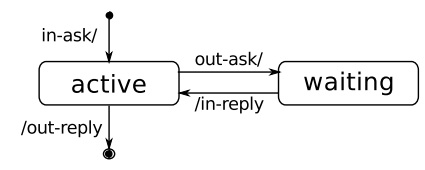

(c) External State Machine

Figure 2.10: Model of a simple e-consultation application in SPACE

The overall activity is called system block. In our example, the local blocks are initiated with a special node denoted as filled circle $(\bullet)$. Pins at sides of building blocks are used to control their behaviour passing tokens of control or data flows along corresponding edges. The white pins represent pins that are used to start (the start and in-ask pins) or to terminate (the out-reply pins) building blocks. The dark pins denote streaming pins, i.e. pins that are used just to pass data objects. In our case, they are ask, reply, out-ask, and in-reply. The pins out-reply and in-ask transmit data objects as well, but these are activating and deactivating pins respectively, and, therefore, are coloured in white. 
Figure 2.10(c) illustrates the ESM for the Simple Chart building block that is a modified UML state machine. The labels of the transitions refer to pins that sit on sides of the corresponding building block used to pass tokens. Thus, pins are used to activate transitions. The slash symbol (/) indicates if a transition is activated by an input (the slash symbol follows the label) or output (the slash symbol preceeds the label) pin.

Similar to functional building blocks, security mechanisms can be expressed as self-contained building blocks. SPACE has been already used for encapsulating security functionality in the form of building blocks [41] validating their correct integration [42]. Additionally, the recent work of Gunawan and Herrmann [43] enables compositional verification of security properties for SPACE models.

\subsubsection{Tools}

MBE promotes the use of modelling for a set of sophisticated tasks of system development. It defines techniques to manipulate produced models, e.g. for simulation, verification, and transformation. These techniques, in turn, must be supported with corresponding tools to enjoy all benefits that are provided by MBE. This section outlines some basic and widely spread tools that enable the practices of MBE.

There are two main languages for meta-modelling, namely MOF (referred to in Section 2.2.1) and Ecore. Recall, that a meta-model or abstract syntax defines the structure of a modelling language, i.e. its constructs, relations, and properties. Ecore is a meta-modelling language used within the Eclipse Modelling Framework (EMF) [44]. The EMF project is a widely used modelling framework that allows engineers to work with modelling languages. EMF provides facilities to define abstract and concrete syntax, and to create editors for custom models and Java code for developed meta-models. MOF is an OMG standard language for meta-modelling that, in particular, defines UML. OMG defines two variants for MOF, namely Essential MOF (EMOF) and Complete MOF (CMOF). CMOF extends EMOF with additional structures. To specify a modelling language using EMOF, an engineer can use the Kermeta tool [45]. Alternatively, KM3 (Kernel Meta-Meta-Model) [46] is a textual language to create meta-models for DSMLs. Meta-models specified in Ecore or MOF can be serialised to an XMI file.

A variety of tools exist to define a concrete syntax for a DSML. For example, Graphiti [47] and GMF [48] are Eclipse-based graphic frameworks that allow developing custom editors. These tools enable automatic generation of a basic editor that can be further refined and tuned. Alternatively, an engineer can define the text representation of a concrete syntax for a modelling language using such tools as Xtext [49]. It provides a language to define grammars and a generator to create parsers and Eclipse-based editors for DSMLs.

A lot of tools are available for modelling with UML: MagicDraw [9], 
Enterprise Architecture [50], Rhapsody [51], to name some main examples. Additionally, EMF provides its own UML2Tool plug-in [52] for defining UML models. Most of the tools mentioned above already support the use of the MARTE profile providing corresponding plug-ins. Moreover, MARTE is implemented in the Eclipse-based Papyrus tool [53]. In our work, we use the MagicDraw tool together with its MARTE plug-in since MagicDraw was used in a European project in which we participated. Besides, we use the Eclipse-based Arctis tool-set [37] to work with the language of the modelbased engineering method SPACE describe in Section 2.2.4.

Atlas Transformation Language (ATL) and Query/View/Transform (QVT) are M2M transformation languages. QVT (QVT Operational) [54] is an imperative language standardised by OMG that allows specifying unidirectional transformations. ATL [55] is a declarative and imperative (hybrid) language developed within EMF. To create transformations using these languages, an engineer needs to write a script. Henshin [56] and EMorF [57] are declarative EMF M2M transformation languages where transformations are specified graphically. There is also an extensive support for M2T transformations. For example, EMF provides Java Emitter Template (JET), Acceleo, and Xpand template-based languages.

\subsection{Ontology Technologies}

An ontology [58] represents knowledge in a particular domain as a set of concepts and their relations. This knowledge is formalised as a logic-based system and described by knowledge representation languages. In particular, we use the Web Ontology Language (OWL2) [59] which is a commonly used and standardised language for creation of large ontologies.

OWL represents an ontology as a sequence of axioms. These axioms describe classes, relations between classes, and their individuals. An OWL class declares the concept of a domain and can be refined by sub-classes. OWL individuals are instances of OWL classes. OWL supports two types of relations. OWL object property defines a relation between two individuals, where one of them plays the role of a domain, and another one plays the role of a value range. In other words, domain defines a subject of a relation, whereas range defines an object. OWL datatype property serves to introduce relations between an individual (domain) and the XML schema datatypes (range) known as XSD (XML Schema Definition). XSD provides such primitive data types as boolean, integer, etc.

The OWL language supports a set of constructs that facilitate management of ontologies. In particular, the OWL language implements the importing feature, which allows relating different OWL ontologies using the owl:import statement. When merging two or more ontologies, it may be the case that these ontologies contain overlapping concepts that have different names, but actually refer to the same things from the reality. Such similar concepts should be related in the merged ontology using the construct 
owl:sameAs. This procedure sometimes is referred as ontologies alignment that is the process of determining correspondences between concepts.

OWL ontologies enable querying of the declared knowledge by combining ontology reasoners (e.g. Pellet or HermiT) and SPARQL querying language [60]. SPARQL 1.1 is a standard query language (recommended by W3C) to execute data queries on top of OWL. It supports yes/no-questions (the $A S K$ query form), a selection which matches a desired pattern (the $S E$ $L E C T$ query form), filtering (the FILTER modifier), sorting (the ORDER modifier), string matching, etc.

To design and manage an ontology, one can use such a tool as Protégé [61]. Since tools developed in this work are Java-based, we exploit the Java OWL API $[62,63]$ to manipulate ontologies (i.e. addition and modification of axioms). To execute SPARQL queries, one can load an ontology into the Protégé tool and use its SPARQL plug-in [64]. In our work, we use Java APIs provided by the widely accepted Jena [65] framework to query ontologies. It provides the SPARQL compliant query engine (among other services) for OWL ontologies.

The OWL standard [66] defines three variants of OWL, namely OWL Lite, OWL DL (Description Logic), and OWL Full. These three sublanguages have different expressiveness and, consequently, different complexity, and, therefore, are used for different purposes. OWL Lite is the simplest variant and allows the user to capture classification hierarchy and constraints with restricted expressiveness. For example, it permits only 0 and 1 as cardinality values. However, it has a simple implementation and comparatively easy to use. In contrast, OWL DL is the most expressive variant. It supports all OWL constructs, but their use is restricted by a set of requirements and rules outlined by W3C [66]. These constraints maintain computational completeness and decidability of this language. OWL Full relaxes constraints of OWL DL and enjoys all capabilities of OWL constructs. The price for this freedom is absence of any computational guarantees.

\section{Ontologies for Security}

A number of ontologies for security have been proposed. For example, Herzog et al. [67] and Fenz and Ekelhart [68] introduce two ontologies that formalise the domain of information security from different aspects; Kim et al. [69] present an ontology for annotating web-services; Karyda et al. [70] propose an ontology to assist reuse of the experts' security knowledge in the area e-government applications. Extended surveys and classification of different security ontologies can be found in works of Blanco et al. [71] and Souag et al. [72] where the authors discuss 28 and 17 security ontologies respectively. In our work, we adopt the ontology presented by Herzog et al. since it is built upon classic components of risk analysis. We continue with a brief description of this ontology.

The core of Herzog et al. ontology [67] consists of six classes. Four of them are concepts related to risk analysis, i.e. asset, vulnerability, threat, 
and countermeasure. The remaining two classes are security goal and defence strategy. Relations between these concepts are defined as follows: an asset can have several vulnerabilities; a threat threatens assets with respect to some security goals; a countermeasure protects assets with respect to security goals by means of defence strategies.

The ontology gives diverse classifications of countermeasures, assets, threats, and vulnerabilities relevant for information security. In particular, the ontology defines 133 countermeasures, 79 assets, 88 threats, and 14 vulnerabilities. The security goal and defence strategy classes are described by a set of individuals. Six individuals are defined for the defence strategy class, namely correction, deflection, detection, deterrence, prevention, and recovery. Fifteen individuals are defined for the security goal class, e.g. confidentiality, integrity, authorisation, and anonymity.

\subsection{Case Study: Metering Infrastructure}

Figure 2.11 depicts an infrastructure called Trusted Sensor Network (TSN) from the smart metering domain. This case study is provided by the MixedMode company that are active in the European SecFutur project [10]. TSN is built of a set of metering devices, database servers, client applications, and a communication infrastructure. The main goal of this system is to measure energy consumption at households and to associate measurements with the clients' data for billing purposes.

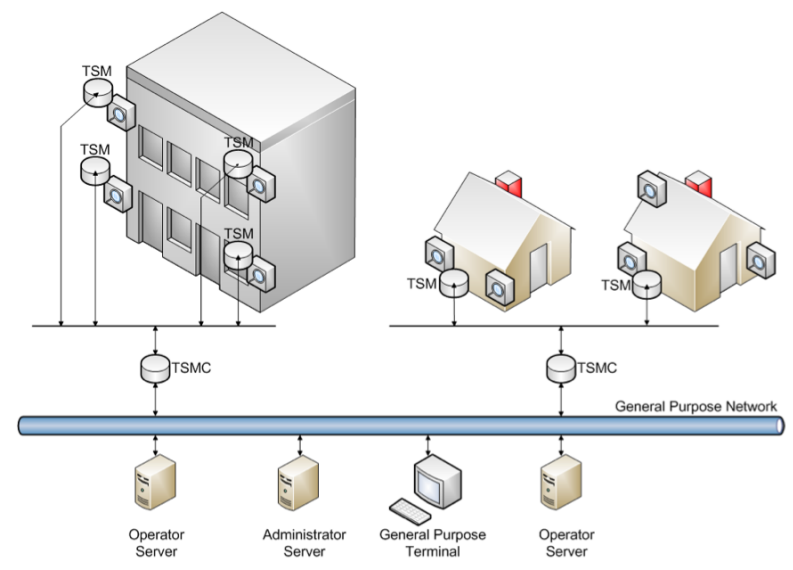

Figure 2.11: Smart metering infrastructure (an overview)

The actual measurement is done by Trusted Sensor Modules (TSMs) consisting of a computing platform and physical sensors. The acquired measurement data is transferred via a local bus from each TSM to a Trusted Sensor Module Collector (TSMC). All measurements collected by TSMCs 
are eventually sent to an operator server through a general-purpose network. Note that the TSMC is also an embedded device, similar to TSM but with more functionality. That is, TSMC and TSM are functional modules that are implemented on the same physical platform.

The overall specification of this case study consists of 11 main scenarios that have a range of diverse security considerations. In this thesis, we focus on the measurement data transfer from TSM to TSMC and from TSMC to an operator server. Consequently, we concentrate on those security issues that concern confidentiality and integrity of the measurement data produced, collected, or stored by the system components. 



\section{Chapter 3}

\section{SEED: Bird's Eye View}

This chapter presents a Security-Enhanced Embedded system Design (SEED) approach, that provides concepts, methods, and tools for dealing with security issues of embedded systems already at the design phase.

\subsection{Introduction to SEED}

SEED addresses the situation when system engineers are not necessarily security experts, and when security experts are not easily accessible to assist system engineers. Overall, the SEED approach rests on three basic principles discussed in the introduction:

- model-orientation,

- domain specialisation, and

- separation of responsibilities and concerns.

Besides these principles, there is another significant design consideration, namely the proposed approach is defined as an increment to existing practices and process models known for embedded system development. These concerns for security-related processes have been revealed by Whit and Harrison [73]. The authors point out that among factors that prevent enforcing security in a systematic way are a lack of security expertise and hesitation of system engineers to commit to follow a new approach that deals with the security aspects due to associated risks.

The SEED approach can be considered in two parts that describe it at two levels of abstraction. These parts are SEED foundation and SEED realisation. The SEED foundation is a generic form of the proposed process ${ }^{1}$ that can be instantiated for different technologies, modelling and formal languages. The SEED foundation defines main activities, involved roles, used

\footnotetext{
${ }^{1}$ The words approach and process are used interchangeably in this thesis.
} 
principles, and their exploitation. The SEED realisation is an implementation of the generic process on a selected set of technologies and languages. In this thesis, the set of languages and technologies selected for one SEED realisation are SPACE, MARTE, and ontology.

This chapter focuses on presenting the generic process, i.e. the SEED foundation. The realisation details are explained in Chapters 4 and 5 where we also illustrate application of the SEED using the smart metering case study.

\subsection{The SEED Foundation}

Figure 3.1 depicts the generic process ${ }^{2}$ of the SEED foundation. It consists of three activities: creation of a system model, capturing of the domainspecific security knowledge, and development of a security-enhanced embedded system. These activities are performed by embedded system engineers or security experts. Thus, the SEED approach acts as a vehicle for communication between the two expert groups. We continue explaining each of the above-mentioned activities.

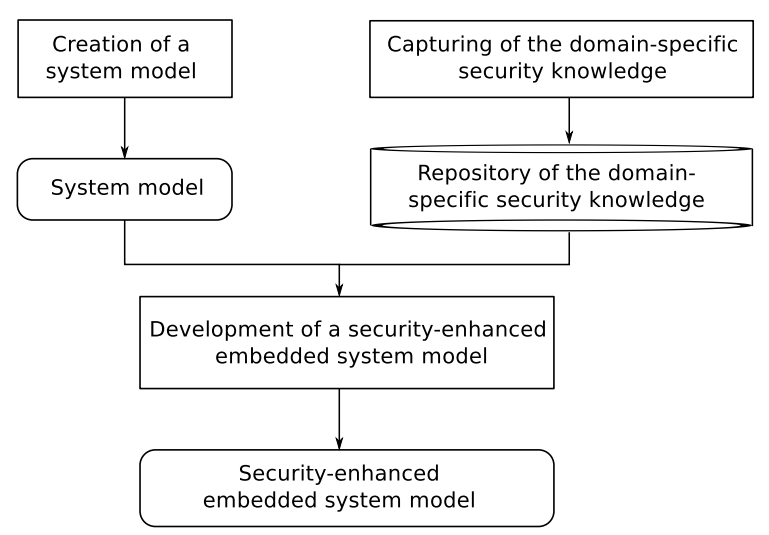

Figure 3.1: Generic process - the SEED foundation

\subsubsection{Creation of a System Model}

The activity of creation of a system model is performed by an embedded system engineer. Figure 3.2(a) depicts a fragment of a simplified embedded system design flow that demonstrates artefacts that are used for the SEED foundation. Note, that this figure does not detail how an embedded

\footnotetext{
${ }^{2}$ It has been developed in cooperation with research partners from the EU FP7 SecFutur project [10].
} 
system engineer produces the artefacts (see the process models described in Section 2.1).

An embedded system engineer starts from system specifications and creates a functional model of a system and a set of models for potential execution platforms. A functional model of a system is a set of modelling elements that describe structural and behavioural aspects of an application, e.g. UML class and activity diagrams respectively. An execution platform model of a system describes an assembly of resources. Each resource provides some services to support execution of an application described as a functional model. Thereafter, an embedded system engineer proceeds to select the best-fitted execution platform. This step includes allocation of elements of a functional model onto available resources, i.e. onto the execution platform model. In other words, allocation is used to establish an association between elements of functional and execution platform models. To sum up, the generic process, i.e. the SEED foundation, uses three artefacts produced while designing an embedded system. These artefacts are a functional model, execution platform model (just platform model from now on), and allocation information.

Hence, SEED is intended to support security analysis when a system engineer has a version of a system design when some decisions about functionality of an embedded system and its hardware/software architecture have been made. At this point, an embedded system engineer has relevant information needed to estimate the system's capabilities to deal with security aspects. In particular, the initial system design is present, valuable objects and actions that influence them are known, and the capacity of a system dedicated for security enforcement of security can be indicated.

\subsubsection{Capturing the Domain-specific Security Knowl- edge}

Capturing (and consequently storing) of the domain-specific security knowledge is an essential step to enable further reuse. This activity is conducted by a domain security expert (just a security expert or a security engineer from now on), i.e. a security expert who has knowledge about an application domain. The main task that a security expert completes as a part of this activity is to describe available security mechanisms. Additionally, a security expert provides other relevant information that is needed for an embedded system engineer to make informed decisions to select a suitable set of security mechanisms.

Figure 3.2(b) illustrates a simplified flow of a security mechanism development adopted in SEED. First, a functional model is created based on some mathematical abstraction, e.g. an algorithm defined as a mathematical object for further analysis. Similarly to an embedded system, a functional model defines behaviour and structural aspects of a security mechanism. Thereafter, a security expert proceeds to implement it. At the 
implementation phase, besides a functional model, a security expert needs some information about execution platform constraints, e.g. to select an implementation language or libraries. Thereafter, the evaluation step allows studying performance aspects of a security mechanism (i.e. the created resource overhead for a provided security level) on selected evaluation execution platforms given a certain workload.

The set of artefacts mentioned above, namely a functional model, execution platform constraints, data about performance evaluation (evaluation platforms, workload, and results of evaluation), constitute the domainspecific security knowledge. Recall that a domain represents an application domain like smart metering devices and set-top boxes in the context of this work. Additionally, the domain-specific security knowledge includes a declaration of security properties provided by a security mechanism. All together, these artefacts give a holistic view on existing security solutions needed to support their integration into an embedded system. For example, an embedded system engineer can study such aspects as: whether a system integrated with a security mechanism still maintains its dedicated functionality in a satisfactory way; or whether an integrated security mechanism fits in the resource-related constraints of an embedded system; or whether security properties of an integrated security mechanism correspond to formulated security requirements of a system.

The domain-specific security knowledge is stored in a repository so that it is available for an embedded system engineer to its further reuse. The domain specialisation of security knowledge is motivated by the following rationale. Security requirements for a particular application depend on potentially present threats that vary based on the nature of an application and deployment environment (i.e. a domain). As a result, the domain-specific security knowledge will have a different set of required security properties and, consequently, associated with them security mechanisms that satisfy these security properties.

\subsubsection{Development of a Security-enhanced Embedded System}

An embedded system engineer carries out the activity of development of a security-enhanced embedded system. The goal is to extend a system model with security features that are retrieved from relevant parts of the domainspecific security knowledge. In particular, this activity of the SEED foundation is built of three basic steps:

- First, a system model is analysed to identify those parts that need security protection. Both functional and execution platform models are subjects of this analysis.

- Second, the security knowledge is consulted to retrieve a set of relevant security properties (based on outcomes of the previous step). 


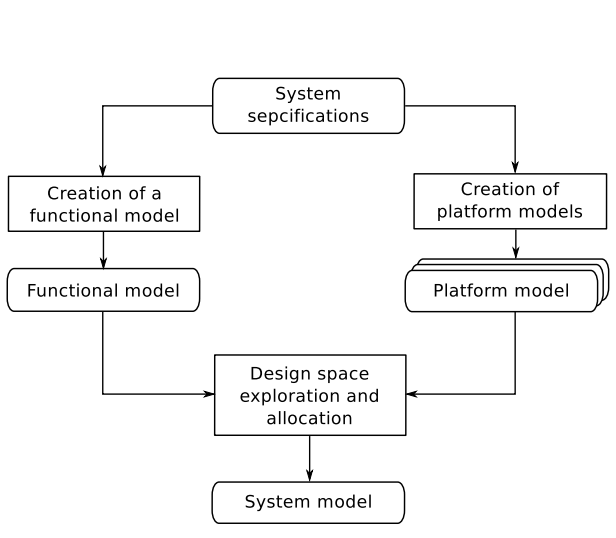

(a) Embedded system

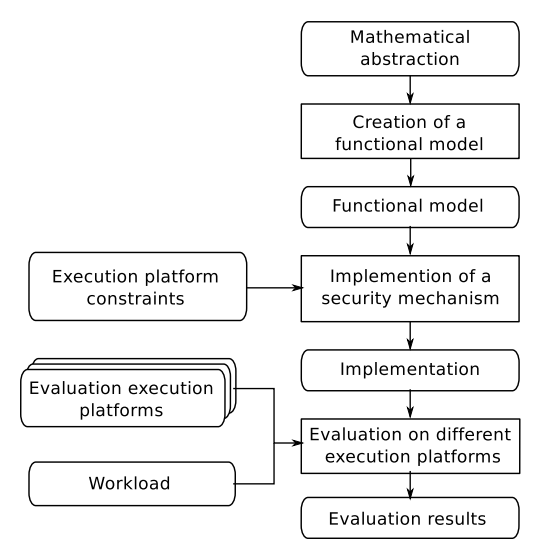

(b) Security mechanism

Figure 3.2: Fragments of simplified design flows

Consequently, a set of security mechanisms that are available in the repository to satisfy identified security properties are also retrieved from the domain-specific security knowledge.

- Finally, since integration of a new feature into an embedded system comes with resource claims, a selected set of security mechanisms is studied with respect to a potentially created resource overhead.

The result of this activity is an embedded system design extended with a suitable set of security mechanisms that meet security goals of a system under development.

In this section, we have described the SEED foundation that presents the generic process intended to support an embedded system engineer and security expert in designing security-enhanced systems. In the next chapters, we describe one instance of the SEED realisation. In particular, we clarify in terms of specific concepts, methods, languages, and technologies how the domain-specific security knowledge is captured by a security expert (in Chapter 4) and how it is applied by an embedded system engineer (in Chapter 5). 



\section{Chapter 4}

\section{Capturing of the Domain-specific Security Knowledge}

This chapter details the "capturing of the domain-specific security knowledge" activity for the SEED realisation. Recall from Figure 3.1, that as a part of this activity, a security expert creates a set of artefacts to describe the existing security mechanisms. This description includes functional model of security solutions, their provided security properties, and information about their performance evaluation. In order to structure and operate with this information, we develop two concepts, namely a Domain-Specific Security Model (DSSM) and a Performance Evaluation Record (PER). These concepts are formalised as a set of ontologies. Additionally, we employ methods and tools from the area of model-based engineering, like modelling languages and transformations, to facilitate the creation and usage of these concepts by embedded system engineers and security experts.

We describe the developed concepts and their support in Section 4.1. Thereafter, Section 4.2 explains processes and tools created to assist a security expert to work with the introduced concepts.

\subsection{Developed Concepts and Artefacts}

As mentioned above, our approach rests on two concepts. DSSM is a concept used by a security expert to describe a security solution; PER is another one that serves to describe results of performance evaluation of a security solution. These concepts are implemented by aligning two technologies, namely UML and ontologies. In the following, Section 4.1.1 and Section 4.1.2 explain the DSSM and PER concepts respectively. 


\subsubsection{Domain-specific Security Model}

A scheme depicted in Figure 4.1 shows the introduced artefacts and their relations. The core artefact is an ontology that we use to define the structure for description of the domain-specific security knowledge. Among studied ontologies for the security domain, the one created by Herzog et al. [67] fits our needs. This ontology is a general information security ontology and was explained in Section 2.3. The ontology created in our work is an adapted Herzog et al. security ontology and is called core security ontology. To facilitate the use of this ontology we define an expert's front-end using the widely accepted UML standard. In particular, the core security ontology is represented as a UML class model. This UML class model serves as a simple tool used by security experts to describe their knowledge about existing security solutions. Therefore, an instance of this model, i.e. object diagram, is actually the captured domain-specific security knowledge and is called Domain-Specific Security Model (DSSM). We transform each DSSM into the OWL syntax and use it to extend the original core security ontology. We refer to an extended ontology as an enriched security ontology. In the following, we continue explaining the mentioned artefacts in more detail.

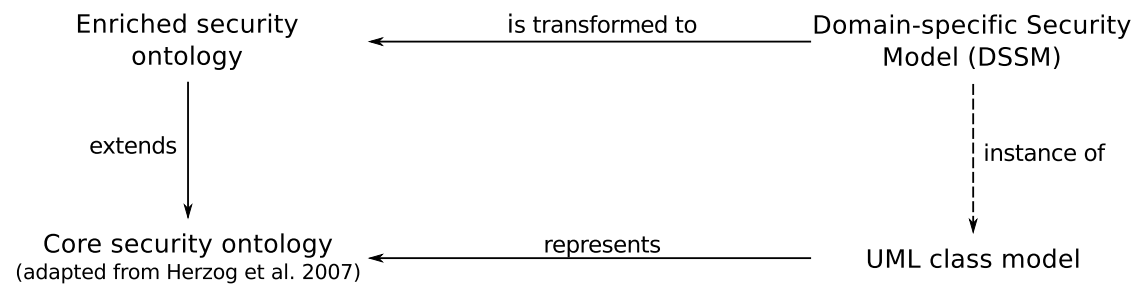

Figure 4.1: DSSM concept and related artefacts

\section{Core Security Ontology}

The core security ontology adapted from the Herzog et al. [67] ontology is depicted in Figure 4.2. The main point of departure arises in order to introduce three new concepts, which are security property, security building block, and domain. Thus, the use of the Herzog et al. ontology has served its purposes outlined by the authors [67], namely as a learning material about the structure of information security and as a framework for developing new detailed security taxonomies. We proceed to describe the adapted ontology used to support the processes of capturing and using the domain-specific security knowledge.

In the core security ontology, we reuse three basic concepts introduced by Herzog et al. [67], namely asset, security goal, and defence strategy. Assets are the "objects of value" of a system that are needed to be protected. In our context, they can be stationary data residing on a physical component or data in transit being transmitted between different components. Other 


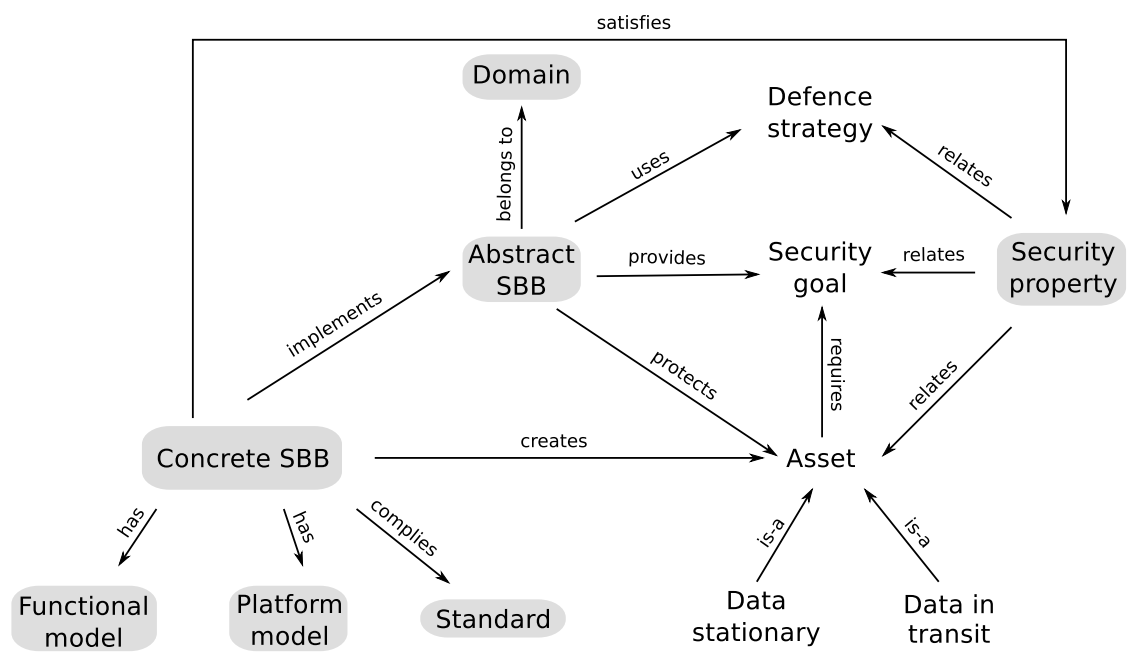

Figure 4.2: Core security ontology fragment

types of assets, e.g. algorithms or Intellectual Properties (IPs), may also be considered and introduced. The protection of an asset leads to the fulfilment of a particular security goal like protecting its confidentiality, integrity, or availability. The countermeasures introduced below follow a certain defence strategy, e.g. preventing attacks or recovering after an attack. For the security goal and for the defence strategy, we reuse all the terms (i.e. individuals) defined in the ontology of Herzog et al.

In addition to these elements, the core security ontology introduces new concepts, which are shown as grey boxes in Figure 4.2. Two of them are abstract and concrete Security Building Blocks (SBBs) replacing the notion of a countermeasure used by Herzog et al. [67]. These refinements enable us to distinguish between more general countermeasures represented by the abstract SBBs and their implementations specified as concrete SBBs. For example, an abstract SBB might refer to a cryptographic hash function as a general method to provide integrity while the different realisations of the hash function (e.g. SHA-1, MD2, or MD5), that are implemented as a piece of code or hardware, are each described by a concrete SBB. With respect to the resource limits of embedded systems, it is important to note that the implementations may have different resource footprints. Each concrete SBB has some functional model and platform model. The latter may be considered as a description of platform components that are required by a concrete SBB for execution. Further, a concrete SBB can comply with some standards, e.g. it may have passed some certification. Another concept introduced by our ontology is the notion of security properties encompassing the three notions: assets, security goals, and defence strategies. Finally, we enrich the ontology with the concept of a domain that represents an 
application domain, e.g. the smart metering domain.

The relations in the core security ontology are defined as follows. Like the countermeasures in the Herzog et al. ontology, an abstract SBB protects an asset, provides some security goals, and uses some defence strategies. We have modified the protects relation for a security goal and a defence strategy used by Herzog et al. [67] since we find that the provides and uses relations reflect more precisely their semantics within our process. In addition to these three relations, an abstract SBB belongs to some application domain. A concrete SBB implements an abstract SBB but, in turn, may create certain assets itself. Therefore, both the assets in the core system and the assets created to realise the concrete SBBs require security goals. For example, the keys in some implementation of a public key cryptography mechanism have to be protected in order to fulfil the confidentiality and integrity goals. Functional and platform models are related to the concrete SBB concept with the has relation. The last relation of the concrete SBB concepts is a comply that relates it to the standard concept. This covers common requirements in engineering of networked embedded systems. In the metering domain, for example, a system will have to fulfil legal calibration requirements following a standard. Finally, a security property relates assets, security goals, and defence strategies, which in the following sections will be referred to by triplets [asset, security goal, defence strategy].

\section{The UML Representation}

To support a security expert in capturing the domain-specific security knowledge that is formalised as the core security ontology, we represent the ontology as a UML class model depicted in Figure 4.3. Each DSSM is effectively an instantiation of the core security ontology. Therefore, we specify a DSSM as an instance of this class model. The security knowledge captured by each DSSM is used to extend our core security ontology presented above with a corresponding set of axioms on relations and individuals. This enables us to use the ontology querying and reasoning services to extract required parts when this knowledge is required for an embedded system engineer. In other words, the class model in Figure 4.3 serves as a language dedicated for capturing knowledge by security experts, i.e. to create DSSMs, while the ontology in Figure 4.2 is a formalism for this language [25].

It is worth mentioning, that the UML model in Figure 4.3 is not a direct transformation of the security ontology from Figure 4.2 since it is not domain knowledge by itself, but rather a tool to capture the knowledge. The model consists of five classes and three relations, which are direct mappings of the elements of the core security ontology. The preserved classes are Asset, AbstractSBB, ConcreteSBB, DataStationary, and DataInTransit. The preserved relations are implements (between Concrete $S B B$ and Abstract $S B B$ ), protects (between Asset and AbstractSBB), and creates (between ConcreteSBB and Asset). The is-a relation from DataStationary and DataInTransit to Asset in the core security ontology is modelled in the form 


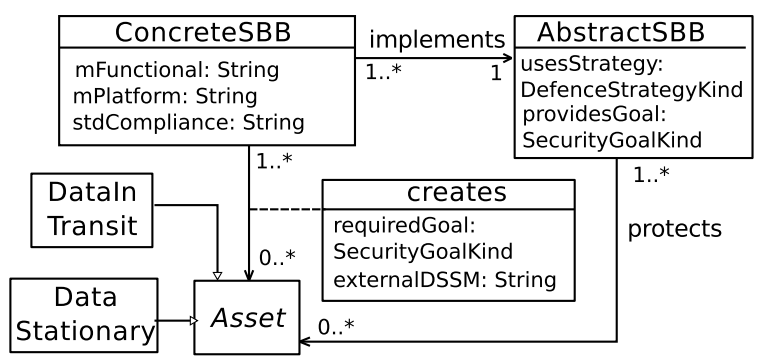

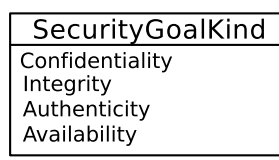

\begin{tabular}{|l|}
\hline DefenceStrategyKind \\
\hline Prevention \\
Detection \\
Recovery \\
Correction \\
\hline
\end{tabular}

Figure 4.3: UML representation of the core security ontology

of generalisations.

Other elements of the core security ontology are specified in a different way. Instances of the security goal and defence strategy classes are represented as the enumerations SecurityGoalKind respective DefenceStrategyKind. The uses relation between the abstract SBB and defence strategy classes in our ontology is represented by the property usesStrategy in the class AbstractSBB. Likewise, the providesGoal property in AbstractSBB replaces the provides relation between the abstract SBB and security goal classes of the core security ontology. Similarly, the functional model, platform model, and standard concepts related to the concrete SBB concept are represented as the mFunctional, mPlatform, and stdCompliance properties of the ConcreteSBB class respectively. Finally, the requiredGoal property in the association class creates represents the relation requires between the created asset and the security goal classes of the ontology.

In contrast, the security property and the domain concepts of the core security ontology are not directly represented in the UML model. This is due to the fact that the triple asset, security goal, and defence strategy already captures the notion of a security property. Therefore, security properties can be directly extracted from a DSSM. Analogously, the domain concept from the core security ontology is represented by the name of a DSSM (i.e. the object diagram).

Besides, the UML class model in Figure 4.3 has one additional property, which does not exist in the ontology, namely externalDSSM in the creates association class. The externalDSSM property refers to other DSSMs. The use of this property is needed if a created asset requires protection provided by security building block that belongs to another known domain.

As mentioned before, a given DSSM is essentially an instance of the UML class model depicted in Figure 4.3. As an example, we depict in Figure 4.4 the UML class diagram for a small fragment of the metering DSSM used for our measurement transfer scenario from Section 2.4. This DSSM contains three assets: StoredMeasurement that represents energy measurements stored on a device; CollectorToServerMsr that represents energy measurements sent from a collector device to an operator server; SensorToMeterMsr 


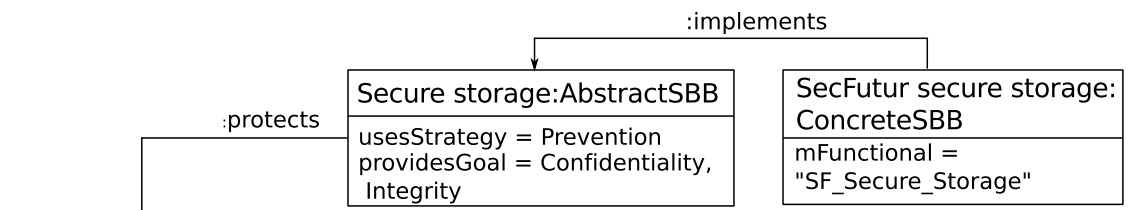

:implements

\begin{tabular}{|c|c|c|c|}
\hline & & :im & nts \\
\hline & protect & $\downarrow$ & \\
\hline$\frac{\downarrow}{\text { StoredMeac }}$ & ament & AbstractSBB & $\begin{array}{l}\text { SecFutur TPM seal: } \\
\text { ConcreteSBB }\end{array}$ \\
\hline DataStat & hary & $\begin{array}{l}\text { usesStrategy = Prevention } \\
\text { providesGoal = Confidentiality } \\
\text { Integrity }\end{array}$ & $\begin{array}{l}\text { mFunctional = } \\
\text { "SF_TPM_Seal" }\end{array}$ \\
\hline
\end{tabular}

:implements
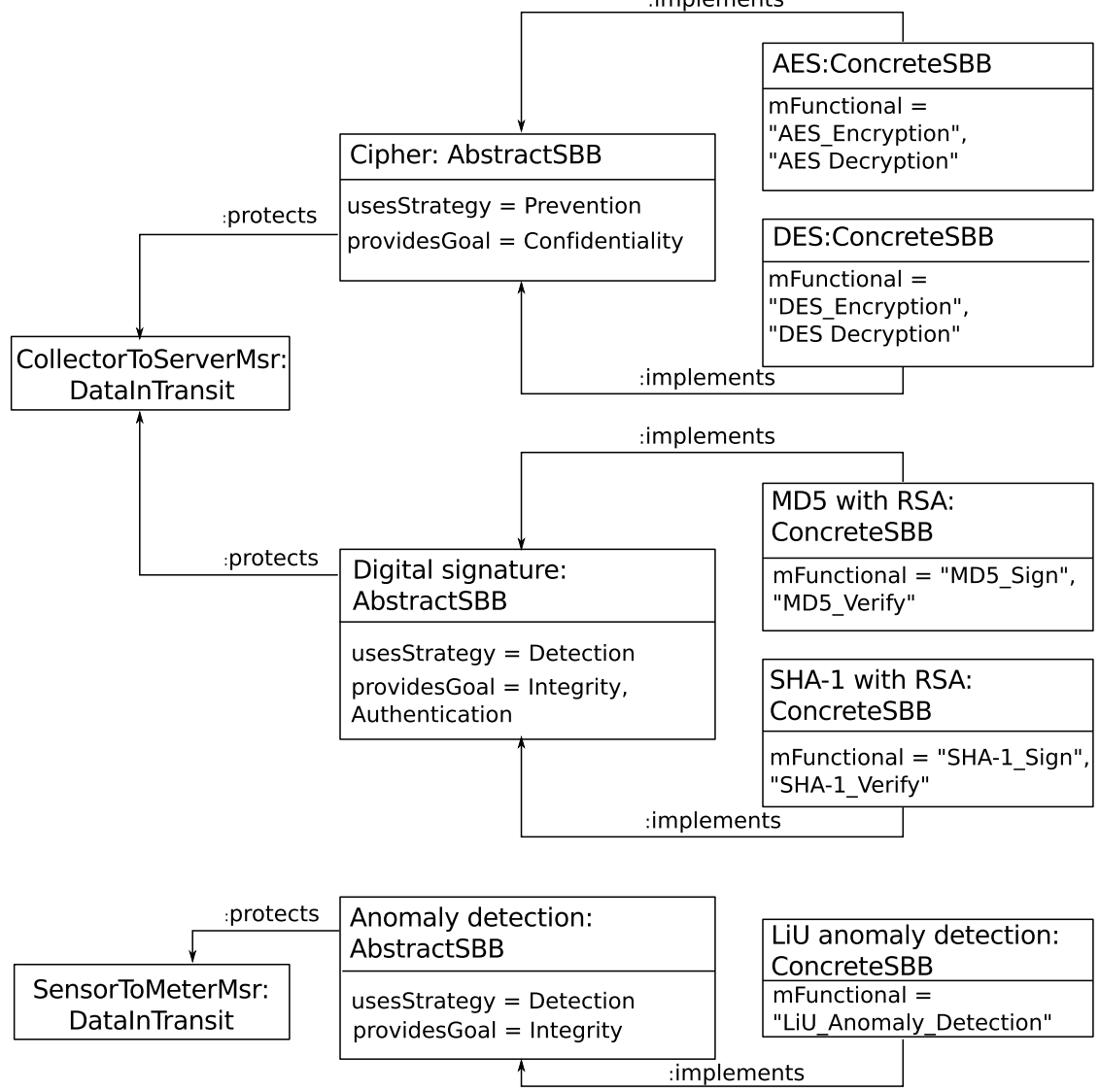

Figure 4.4: Fragment of the metering DSSM

that represents energy measurements sent from a sensor to a metering device. These assets may be protected by five abstract SBBs: Secure stor- 
age and Tamper evident seal that provide confidentiality and integrity for the StoredMeasurement asset; Cipher that provides confidentiality for the CollectorToServerMsr asset; Digital signature that provides integrity and authentication for the CollectorToServerMsr asset; and Anomaly detection that provides integrity for the SensorToMeterMsr asset. Seven concrete SBBs implement these abstract SBBs. Each abstract SBB in Figure 4.4 is supported by one or two implementations. In general, one abstract SBB can be implemented by several concrete SBBs. For example, the DES and AES concrete SBBs implement the Cipher abstract SBB. These concrete SBBs have one or a pair of functional models (see the mFunctional slot). In this study and in this SEED realisation, they are Arctis blocks for encryption (AES_Encryption and DES_Encryption respectively) and decryption (AES_Decryption and DES_Decryption respectively).

\section{Enriched Security Ontology}

In the following, we explain the last artefact introduced in Figure 4.1, namely the enriched security ontology.

Each DSSM is transformed into the OWL syntax (outlined in Section 2.3) extending the core security ontology, as it is depicted in Figure 4.1. In other words, these DSSMs are used to populate the core security ontology. In turn, each DSSM is encapsulated into a separate ontology, called [domain name] security ontology that imports (using the owl:import construct) the core security ontology. We refer to the merge of all domain security ontologies obtained from DSSMs as the enriched security ontology. This modular structure of the enriched security ontology facilitates its management (e.g. its update). Figure 4.5 shows the described dependencies of the introduced ontologies. In this figure, Domain 1 and Domain $n$ represent application domains, e.g. metering devices and set-top boxes domains respectively.

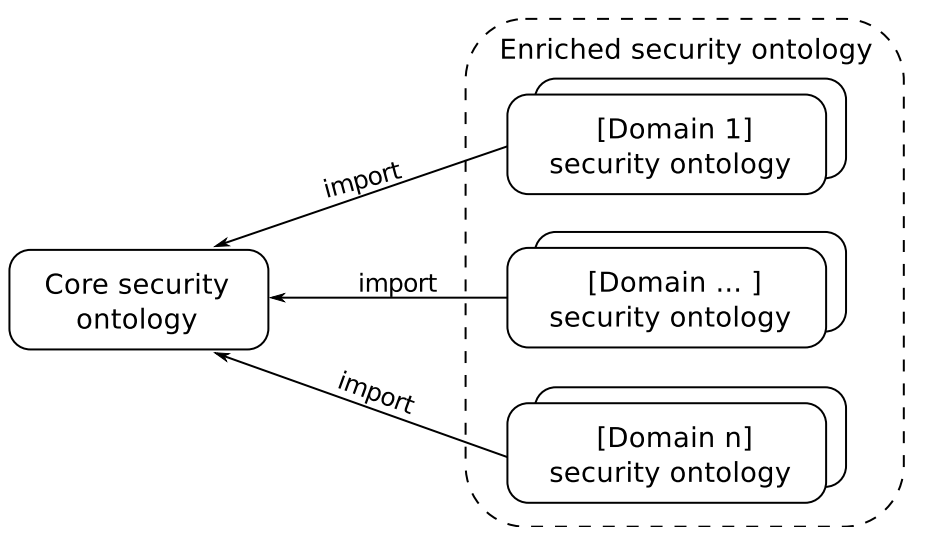

Figure 4.5: Enriched security ontology

The task of updating the enriched security ontology with the knowledge 
captured by a newly created DSSM is implemented as transformation of elements of a DSSM and their relations into corresponding set of axioms on classes, relations, and individuals. We use model-to-text transformation techniques and tools, like Acceleo [74], to realise this transformation. Afterwards, these axioms are added into the corresponding [domain name] security ontology.

All objects of the metering DSSM in Figure 4.4 are added into the enriched security ontology as individuals of the corresponding concepts of the core security ontology from Figure 4.2. Thereafter, additional axioms are added that transform instances of the protects, implements, and creates associations from a DSSM into corresponding (OWL) object properties. The usesStrategy and providesGoal attributes are transformed into triples of [object, object property, subject] where an object is an instance name of the AbstractSBB class, an (OWL) object property corresponds to the uses and provides relations respectively, and a subject is a value of the attribute. The requiredGoal attribute is transformed into analogous triples where an object is an instance name of the Asset class, an (OWL) object property corresponds to the requires relation, and a subject is a value of the attribute. Such attributes as mFunctional, mPlatform, and stdCompliance are approached in a similar way. The difference is that an (OWL) object property is replaced by a $(\mathrm{OWL})$ data property since values of these three attributes are strings.

\subsubsection{Performance Evaluation Record}

Figure 4.6 depicts a scheme that shows the defined artefacts and their relations. This scheme follows a similar pattern as presented in the previous section. The core artefact is an ontology that describes concepts and relations of the performance evaluation domain. This ontology is called core evaluation ontology. Differently from the core security ontology, a UML profile is designed as a representation front-end for the core evaluation ontology. This profile is called General Evaluation Model (GEM). An expert defines an evaluation context as a set of UML models of different types and annotates their elements using stereotypes of the designed profile. These UML models are called Performance Evaluation Record (PER). Further, these models are transformed into the OWL syntax and used to extend the initial core evaluation ontology forming an enriched evaluation ontology.

The infrastructure in Figure 4.6 can be used to capture results of performance evaluation of any building block that realises extra-functional properties, e.g. security, safety, and real-time. Thus, security is just one example of such extra-functional properties. Therefore, we use generic terms in this section, i.e. the SBB term introduced in the previous section is replaced by the Reusable Building Block $(R B B)$ concept. We use examples of security RBBs (i.e. SBBs) to illustrate some introduced concepts ${ }^{1}$.

\footnotetext{
${ }^{1}$ In the rest of the thesis (except this section), we avoid using the term RBB. However,
} 


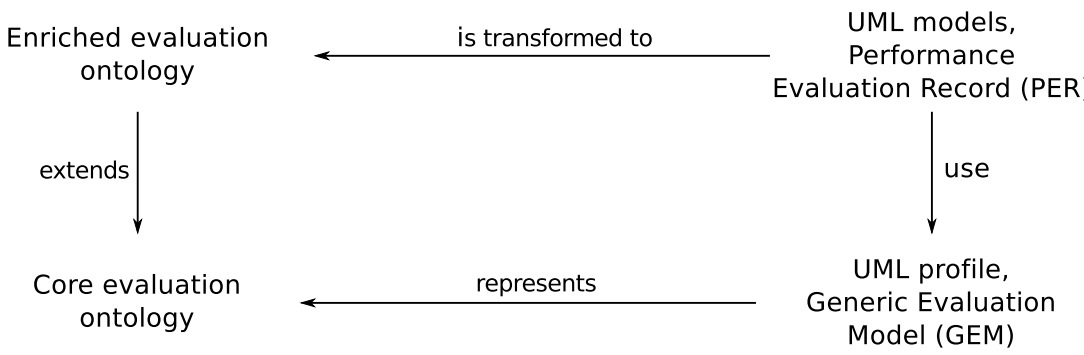

Figure 4.6: PER concept and related artefacts

In the following, we continue explaining in more detail the mentioned artefacts.

\section{Core Evaluation Ontology}

Figure 4.7 depicts an ontology developed in this study in order to capture results of performance evaluation of RBBs called core evaluation ontology. To build the core evaluation ontology, we use the knowledge about the structure of the analysis domain obtained from various sources. They are mainly the MARTE GQAM profile explained in Section 2, and the published research and industrial papers where performance aspects of different RBBs are studied, e.g. such works published by Nadeem and Javed [75] and Preissig [76] where performance characteristics of security solutions are investigated. We proceed to describe elements of the core evaluation ontology depicted in Figure 4.7.

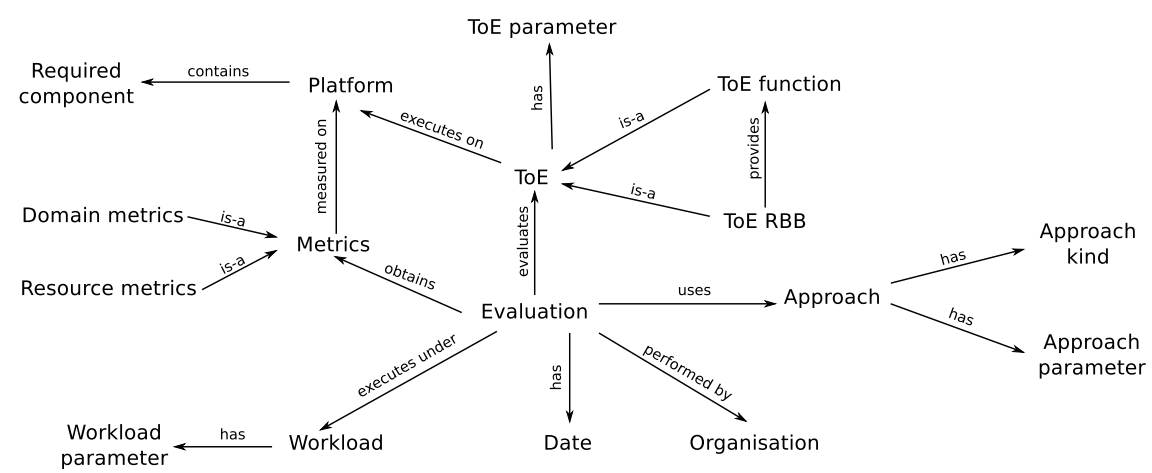

Figure 4.7: Core evaluation ontology

Evaluation is a central concept of the core evaluation ontology. It relates four other concepts that indicate constituents of any evaluation procedure.

a reader should understand that the term SBB can be replaced by $\mathrm{RBB}$ when it comes to discussions of PER and related concepts (e.g. in Section 4.2 and 5.5). 
They are Target of Evaluation (ToE), approach, workload, and metrics.

$T o E$ refers to an $\mathrm{RBB}$ that is under performance evaluation. There are two sub-classes for the ToE concept, namely ToE RBB and ToE function. A ToE can be characterised by a set of parameters introduced by the $T o E$ parameter concepts, e.g. the key size for an encryption RBB. The platform concept denotes an evaluation platform used for performance analysis. We create the required component concept to introduce those components of an evaluation platform that are significant for a considered ToE to obtain the captured performance results. For example, it can be a particular instruction set exploited by a ToE implementation.

The approach concept denotes the description of the used evaluation method. Each approach can be characterised by its kind and approach parameters. The approach kind concept defines the type of the evaluation method. It has such individuals as simulation, emulation, or analytical analysis. The workload concept introduces the workload used during the performance evaluation, e.g. a sequence of triggering events, or an amount of data sent over a channel. Similarly, a workload can be parameterised using the workload parameter concept.

The metrics concept defines a set of metrics adopted for the performance evaluation. These metrics can be of two categories as denoted by its sub-classes. The first category (the resource metrics concept) describes the resource footprint created by ToE (e.g. execution time). The second category (the domain metrics concept) refers to the obtained indicators that characterise the quality of extra-functional properties, e.g. the security level provided by a ToE. The presence of these two categories reflects the fact that for selection of suitable RBBs both resource footprint and quality of service indices play a significant role.

The following relations are defined in the core evaluation ontology. Any evaluation evaluates some ToE, executes under some workload, uses some approach, and obtains some metrics. A ToE RBB can provide some ToE functions. Any ToE executes on some evaluation platform. A platform may contain some required components. Any obtained metrics are measured on some platform. A ToE, approach, and workload have some ToE parameters, approach parameters, and workload parameters respectively. Additionally, an approach can have some approach kind. Finally, each evaluation has some date and performed by some organisation.

\section{The UML Representation}

In this section, we define a profile used by an expert in order to capture performance evaluation results called Generic Evaluation Model (GEM). This profile is depicted in Figure 4.8 .

The GEM profile consists of nine stereotypes and nine relations which are direct mappings of the elements of the core evaluation ontology. The preserved classes are gemToE, gemToE_RBB, gemToE_Function, gemEvaluation, gemApproach, gemWorkload, gemMetrics, gemPlatform, and gem- 


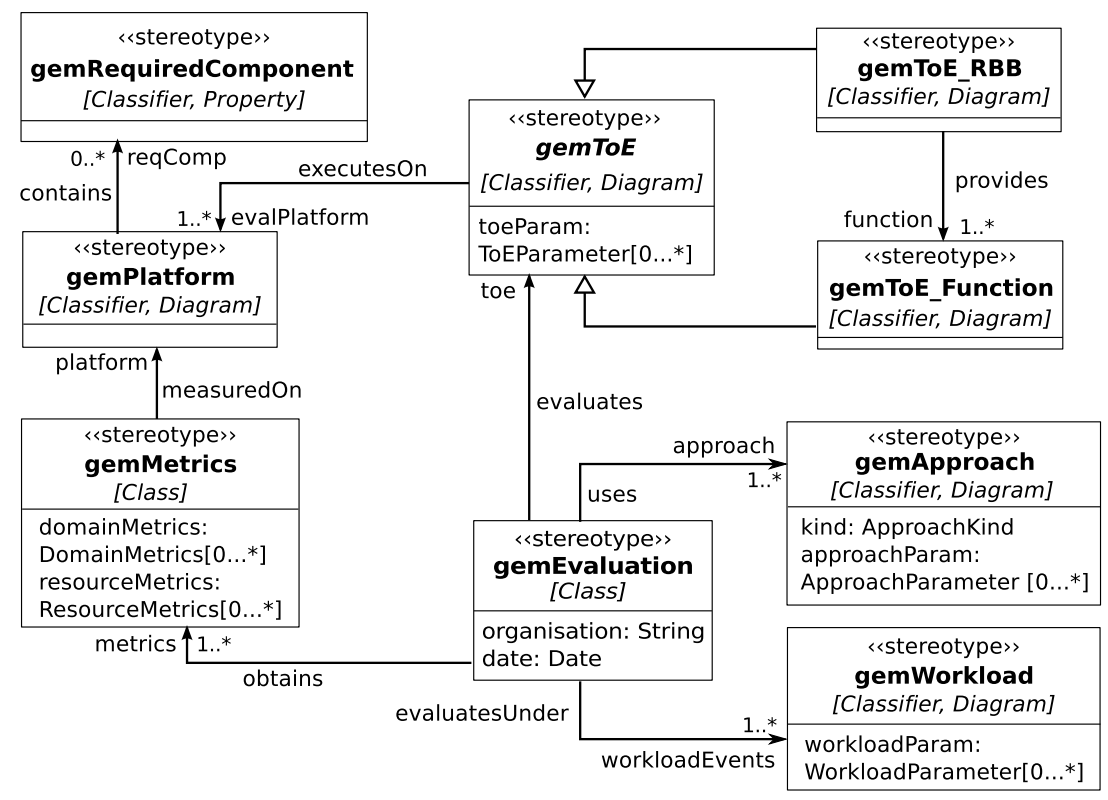

Figure 4.8: GEM UML profile to capture performance evaluation results

RequiredComponent. The preserved relations are uses, evaluatesUnder, obtains, and evaluates which relates gemEvaluation with gemApproach, gemWorkload, gemMetrics, and gemToE respectively. Other directly mapped relations are measuredOn between the gemMetrics and gemPlatform stereotypes, contains between gemPlatform and gemRequiredComponent, executesOn between gemToE and gemPlatform, and provides between gemToE_RBB and gemToE_Function. The is-a relations from gemToE_RBB and gemToE_Function to gemToE in the core evaluation ontology are modelled as generalisations in GEM.

Other elements of the evaluation ontology are specified in a different way. Individuals of the approach kind class are represented as an ApproachKind enumeration (not shown). The has relation between the ToE and ToE parameter classes in our ontology are represented by the property toeParam in the class gemToE. Likewise, the approachParam property in gemApproach replaces the has relations between the approach and approach parameter concepts of the core evaluation ontology. The same logic applies for the workloadParam property. Finally, the sub-classes of the metrics concept are represented as corresponding tags of the gemMetrics stereotype, namely domainMetrics and resourceMetrics.

In the following, we explain what UML models can be annotated with the stereotypes from GEM, and what MARTE packages are reused. We conclude this section with an example illustrating a possible use of the GEM profile. 
gem ToE, gemApproach, and gem Workload can be used to annotate either a complex or a very simple UML model of a corresponding constituent. The level of detail does not play a significant role for the GEM profile. Nevertheless, the richer these models are the more informed decisions can be made by an embedded system engineer when selecting RBBs.

In order to model an evaluation platform, i.e. the gemPlatform stereotype, we use a UML class model annotated with stereotypes from the HRM MARTE package. An execution platform can describe resources that take a variety of forms, e.g. hardware, software, or logical resources. In this study, we consider only hardware components. However, the general concept is scalable to include other forms of resources for analysis. Additionally, we employ the MARTE NFP types to specify values of all parameters and metrics tags defined in GEM.

The MARTE GQAM profile (outlined in Section 2.2) allows bridging the gap between model-driven engineering and existing formalisms and tools for analysis. Thus, it significantly helps the domain (in our case, security) experts to design their evaluations. In order to demonstrate how our profile can be used to capture performance evaluation results modelled in GQAM, we identify the correspondence between the stereotypes and tags of GEM and GQAM shown in Table 4.1.

Note that our profile is not restricted to capturing results only when GQAM or its refinements are used. For example, results presented by Preissig [76], that are obtained through a traditional approach, can also be described by GEM (as we will show later in this section). However, GQAM can facilitate this task, since the mapping identified in Table 4.1 can be automated as a transformation directly feeding relevant data into GEM.

GEM is a general UML profile that does not target any concrete extrafunctional domain. The same statement is applicable for the core evaluation ontology. Therefore, a domain expert should refine some of its concepts to tailor it to a certain domain. In particular, an expert needs to extend the ToEParameter, DomainMetrics, and ResourceMetrics stereotypes. Figure 4.9 shows such a refinement for the security domain as an example where a cipher and anomaly detection RBBs are considered. ToEParam_Anomaly and ToEParam_Cipher refine the ToEParameter concept. According to these refinements, an anomaly detection mechanism can be characterised by the number of clusters (numberOfClusters), while a cipher building block can be characterised by its key size (keySize) and cipher mode (cipherMode). Quality of service metrics for an anomaly detection are detection rate and false positive rate (see DM_Anomaly) and for a cipher they are resistance to attacker's capabilities in terms of its skill, motivation, and duration of the attack (see DM_Cipher). Finally, a pair of resource metrics are considered for these two security RBBs, namely bandwidth and energy (see $R M_{-}$Anomaly) for an anomaly detector and the used memory and data rate (see RM_Cipher) for a cipher.

A description of actual performance evaluation results captured when 
Table 4.1: Correspondence between our GEM and (MARTE) GQAM

\begin{tabular}{|c|c|}
\hline GEM & GQAM \\
\hline gemToE & The GaScenario stereotype \\
\hline toèParam & $\begin{array}{l}\text { No direct mapping. } \overline{\mathrm{It}} \text { can be mappe } \overline{\mathrm{d}} \\
\text { to some variables declared in the con- } \\
\text { textParam tag (the GaAnalysisContext } \\
\text { stereotype) where sourceKind is defined as } \\
\text { required (req). }\end{array}$ \\
\hline gemPlatform & The GaResourcePlatform stereotype \\
\hline gemRequiredComponent & $\begin{array}{l}\text { Any element from a GaResourcePlatform } \\
\text { model can be annotated with this stereo- } \\
\text { type. }\end{array}$ \\
\hline gemApproach & Not represented. \\
\hline kind $\bar{d}^{-1}-\cdots$ & $\begin{array}{l}\text { Not represented. } \overline{\text { It }} \text { is } \overline{\text { defined }} \overline{\text { by }} \overline{\text { an }} \overline{\text { anal }}-\overline{-} \\
\text { ysis formalism that underlies the GQAM } \\
\text { model. }\end{array}$ \\
\hline$\overline{\text { approach }} \overline{\mathrm{P}} \overline{\mathrm{aram}}$ & $\overline{\text { Not }} \overline{\text { represented. }}-\overline{-}-\cdots-\cdots-\cdots-\cdots$ \\
\hline 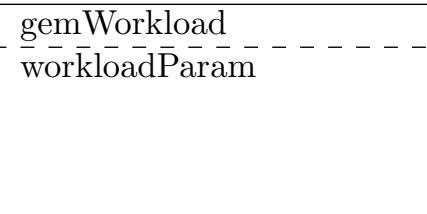 & $\begin{array}{l}\text { The GaWorkloadEvent stereotype } \\
\text { No direct mapping. } \\
\text { to some variables declared in the con- } \\
\text { textParam tag where sourceKind is defined } \\
\text { as required (req). }\end{array}$ \\
\hline gemMetrics & $\begin{array}{l}\text { No direct mapping. It can be mapped to } \\
\text { any declared variable where sourceKind is } \\
\text { set to calculated (calc), estimated (est), or } \\
\text { measured (msr). }\end{array}$ \\
\hline$\overline{\text { resourcēMetrics }} \overline{-} \overline{-}$ & $\begin{array}{l}\text { No direct mapping. } \overline{\text { It }} \text { can be mapped } \\
\text { to some variables declared as tags of the } \\
\text { GaStep stereotype. }\end{array}$ \\
\hline$\overline{\text { domainMetrics }}{ }^{-}-\bar{M}^{-}---$ & $\begin{array}{l}\text { No direct mapping. These variables are } \\
\text { usually defined by domain experts and in- } \\
\text { troduced as a part of the contextParam } \\
\text { tag. }\end{array}$ \\
\hline
\end{tabular}

the GEM profile is used is called Performance Evaluation Record (PER). Figure 4.10 depicts an example of a PER used in development of the smart metering application from Section 2.4. The DES_Evaluation class annotated as "gemEvaluation" shows that an evaluated RBB is DES_Encryption where DES_Metrics is a set of the obtained metrics. The DES_Encryption RBB has configuration parameters specified in DES_Settings and is executed on the TMS320C6211 chip. Besides, TMS320C6211 is annotated with the gemRequiredComponent stereotype and, therefore, will be treated as a platform constraint for DES_Encryption in further analysis. 


\begin{tabular}{|c|}
\hline $\begin{array}{c}\text { «ToEParameter» } \\
\text { ToEParam_Cipher }\end{array}$ \\
\hline $\begin{array}{l}\text { keySize: NFP_DataSize } \\
\text { cipherMode: CipherModeKind }\end{array}$ \\
\hline
\end{tabular}

\begin{tabular}{|c|}
\hline \multicolumn{1}{|c|}{ «TToEParameter» } \\
ToEParam_Anomaly \\
\hline $\begin{array}{l}\text { numberOfClusters: } \\
\text { NFP_Integer }\end{array}$ \\
\hline
\end{tabular}

\begin{tabular}{|l|}
\hline \multicolumn{1}{|c|}{ ¿ DomainMetrics» } \\
DM_Cipher \\
\hline skill: String \\
motivation: String \\
duration: NFP_Duration
\end{tabular}

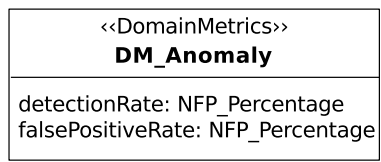

«८ResourceMetrics〉》 RM_Cipher

usedMemory: NFP_DataSize dataRate: NFP_DataTxRate

\begin{tabular}{|c|}
\hline \multicolumn{1}{|c|}{$\begin{array}{c}\text { «ResourceMetrics» } \\
\text { RM_Anomaly }\end{array}$} \\
\hline $\begin{array}{l}\text { bandwidth: NFP_DataTxSize } \\
\text { energy: NFP_Energy }\end{array}$ \\
\hline
\end{tabular}

Figure 4.9: The refinement of the GEM profile for the security domain
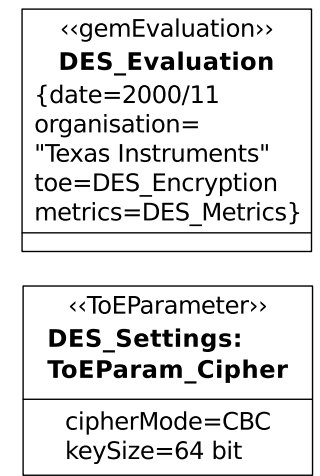
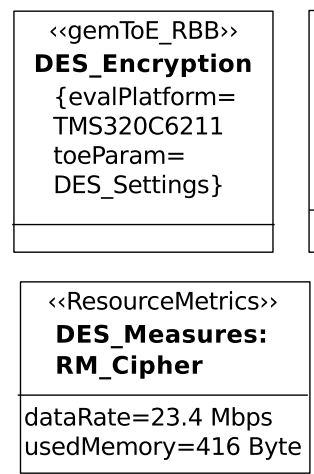
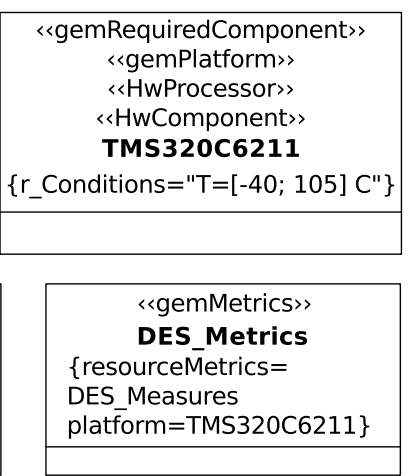

Figure 4.10: Fragment of the security evaluation record for the DES RBB

\section{Enriched Evaluation Ontology}

In the following, we explain the last artefact introduced in Figure 4.6, namely the enriched evaluation ontology.

Each refinement of the GEM profile for an extra-functional domain (e.g. as one depicted in Figure 4.9) is transformed into a separate ontology called [domain name] evaluation ontology that imports the core evaluation ontology enriching it with additional concepts from this domain, e.g. refinements of the ToE parameters, domain, and resource metrics concepts. Since each PER (e.g. as one depicted in Figure 4.10) is essentially an instance of the refined GEM profile, we transform it into axioms on individuals and call it [domain name] evaluation record ontology. The transformation is approached similar to one outlined in Section 4.1.1. For example, a class annotated with the ToE stereotype becomes an individual of the ToE class. A [domain name] evaluation record ontology imports a corresponding [domain name] evaluation ontology. We refer to a collection of [domain name] evaluation record ontologies as an enriched evaluation ontology. Figure 4.11 shows the above introduced ontologies and their dependencies. In this figure, Domain 1 and Domain $n$ represent extra-functional domains, e.g. Domain 1 can be exemplified as the security domain, and Domain $n$ as the safety domain. 


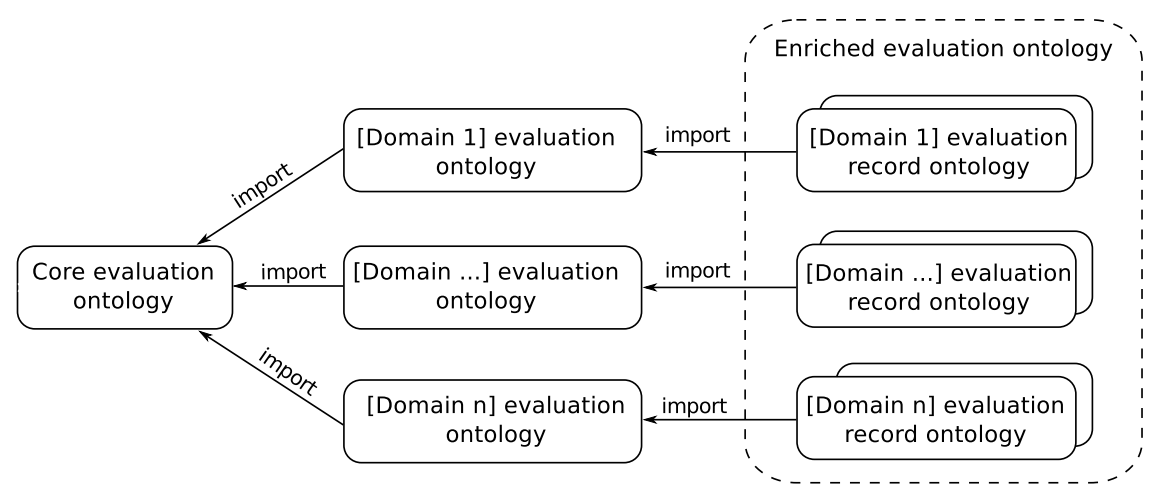

Figure 4.11: Enriched evaluation ontology

\subsection{Capturing Security Knowledge}

In this section, we explain the proposed process for capturing of the domainspecific security knowledge using the DSSM and PER ${ }^{2}$ concepts.

The starting point of the creation of DSSMs is to decide on a domain. The DSML theory inherently leaves the notion of a domain flexible. Hence, it is up to security experts to decide what kind of a domain a DSSM will describe. Typically, we consider application domains (e.g. metering devices, set-top-boxes, banking access terminals), which can be characterised by a different set of assets and a specialised set of security solutions. Domains can be in some relations, e.g. domains can overlap or one domain can be a part of another one. Note that the closer a selected domain is tailored to a type of a system, the more specialised and detailed solutions it contains (i.e. the set of assets and concrete SBBs). For example, both communication and metering DSSMs may be applied for our smart metering devices case study described in Section 2.4, but obviously the communication DSSM will contain such general assets as "message" and "acknowledgement", while the metering DSSM operates with "measurement" as an asset.

The process of DSSM creation is depicted in Figure 4.12. It starts with three activities: Create a DSSM, Create functional models for concrete $S B B s$, and Create PERs for concrete SBBs. The Create a DSSM activity includes definition of assets, abstract SBBs with their goals and strategies, and concrete SBBs omitting the definition of their functional and platform models (i.e. the mFunctional and mPlatform slots of the ConcreteSBB class depicted in Figure 4.3). Thus, the outcome of this activity is a definition of the DSSM with place holders for concrete SBBs. Functional models of each concrete SBBs are created during the Create functional models for concrete $S B B s$ activity. A security expert creates, e.g. Arctis models of the concrete SBBs. The third activity, i.e. Create PERs for the concrete $S B B s$, is

\footnotetext{
${ }^{2}$ In this section, we start again to use the term (concrete) SBB instead of RBB when discussing PER and related concepts shifting the focus back to security.
} 
concerned with a description of the performance evaluation results in the form of PERs. Note that as a part of each PER a security expert defines a platform used for an evaluation of a concrete SBB. This platform model serves as a platform model associated with a concrete SBB (the mPlatform slot) as it will be shown later.

The order of the three activities described above is undefined, since it does not play a significant role for our process. Thus, if Arctis models of the considered concrete SBBs exist (e.g. they are available in the Arctis library of building blocks [38]), the corresponding activity can be omitted. Similarly, the activity Create PERs for concrete $S B B$ s can be omitted. The absence of any of the two types of artefacts produced by these activities (i.e. functional models for concrete SBBs or their evaluation records) will simply disable the corresponding type of analysis.

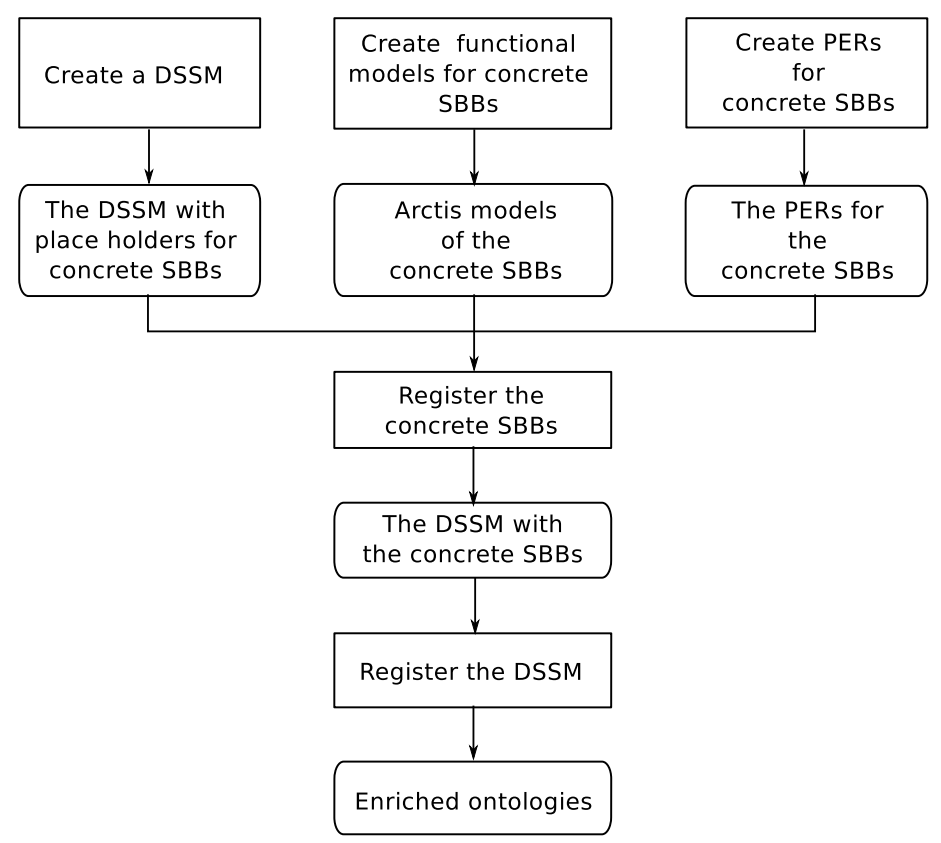

Figure 4.12: The process for creation of DSSMs

The next activity is Register the concrete $S B B$ s that allows associating the created functional (Arctis) models and PER models of concrete SBBs with the corresponding elements of the DSSM. In particular, the functional Arctis model is bound to the mFunctional; the evaluation platform model from the created PER indicated with the gemPlatform stereotype is bound to the mPlatform slot. When all concrete SBBs of the created DSSM have been bound with the functional and PER models, the DSSM can be registered.

The main outcome of the Register the DSSM activity is two ontologies. 
The first ontology is a [domain name] security ontology derived from the DSSM that is a part of the enriched security ontology as explained in Section 4.1.1. The other ontology is a [domain name] evaluation record ontology derived from the PER that is a part of the enriched evaluation ontology as explained in Section 4.1.2. Note that the enriched security and evaluation ontologies are two independent ontologies that can be used separately from each other. In our work, we align them employing the owl:sameAs construct (see Section 2.3) as follows: PlatformModel owl:sameAs Platform, FunctionalModel owl:sameAs ToE.

It is worth noting that this architecture is modular. For example, the activity Create PERs for concrete $S B B$ s can be replaced by any other activity that allows preparing concrete SBBs for a desirable type of analysis. This will require creation of the needed infrastructure (e.g. ontologies and profiles) and adjustment of the alignment.

When updating the enriched ontologies with new knowledge, the important question of maintaining their consistency arises. In particular, an obvious problem when updating the enriched security ontology is its pollution with concrete SBBs that have different names but refer to the same implementation ${ }^{3}$. The unique name assumption of an ontology says that entities with different names refer to different elements of the real world. The OWL language has two constructs to express this assumption, namely owl:sameAs or owl:differentFrom, that assert that two or more given entities refer to the same or to different elements of the real world respectively. We use the latter construct each time a new concrete SBB is added into the enriched security ontology. However, it may be the case that security experts will populate the enriched security ontology with concrete SBBs that actually refer to the same implementation. This situation can be resolved by the owl:sameAs construct that states that two or more individuals refer to the same element of the real world. However, some additional support may be needed to ensure that two (or more) concrete SBBs under different names are equal implementations. We envisage that techniques from the area of model comparison or models diff (applied to functional and platform models of concrete SBBs) can be employed to address the mentioned issue. For example, Selonen [77] and Bendix and Emanuelsson [78] have a survey about existing model comparison methods for UML models. Besides, a set of tools exist to implement model comparison, e.g. EMF Compare [79]. The exploitation of these techniques goes beyond the scope of this work. We consider it as a further enhancement of our tool support. In the rest of this section, we outline a developed tool that supports the above process of DSSM creation.

As mentioned earlier, we use the MagicDraw tool [9] as an integration environment. To create DSSMs and PERs, we use the standard functionality provided by MagicDraw. At the same time, functional models of concrete SBBs are created in the Arctis tool [37]. In order to bind these two tools

\footnotetext{
${ }^{3}$ The trivial case, i.e. two entities with the same names, is not possible.
} 
supporting the process of DSSM creation, we have developed a SEED MagicDraw plug-in. In particular, this plug-in assists the creation of DSSMs by supporting the following activities of the process depicted in Figure 4.12:

- Creation of a DSSM: the plug-in prepares an environment for a security expert, i.e. it creates a MagicDraw project and loads the class model depicted in Figure 4.3.

- Registration of concrete SBBs: the plug-in provides an interface (shown in Figure 4.13) for binding functional model and platform model elements of concrete SBBs with corresponding Arctis and MARTE (from PER) models.

- Registration of a DSSM: the plug-in executes transformation of the DSSM and PER to a set of axioms and adds them into the enriched ontologies. Additionally, the plug-in can be used to upload the created DSSM and PER to a library (local or public) for its further use.

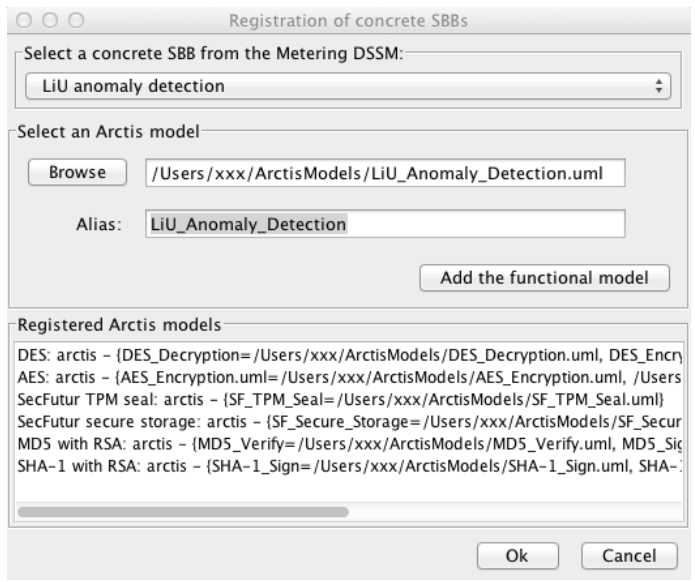

Figure 4.13: Registration of concrete SBBs tool (the user interface)

This chapter has explained capturing of the domain-specific security knowledge. For this purpose, we have introduced two concepts, namely the DSSM and PER concepts. The process followed by a security expert, that exploits DSSMs and PERs, has been described. In the next chapter, we describe how an embedded system engineer can apply this knowledge to select a suitable set of security solutions to be integrated into a system. 


\section{Chapter 5}

\section{Application of the Domain-specific Security Knowledge}

This chapter explains the "Development of a security-enhanced embedded system model" activity for the SEED realisation. In particular, we focus on the process of application of the domain-specific security knowledge captured using DSSMs and PERs. This process is depicted in Figure 5.1.

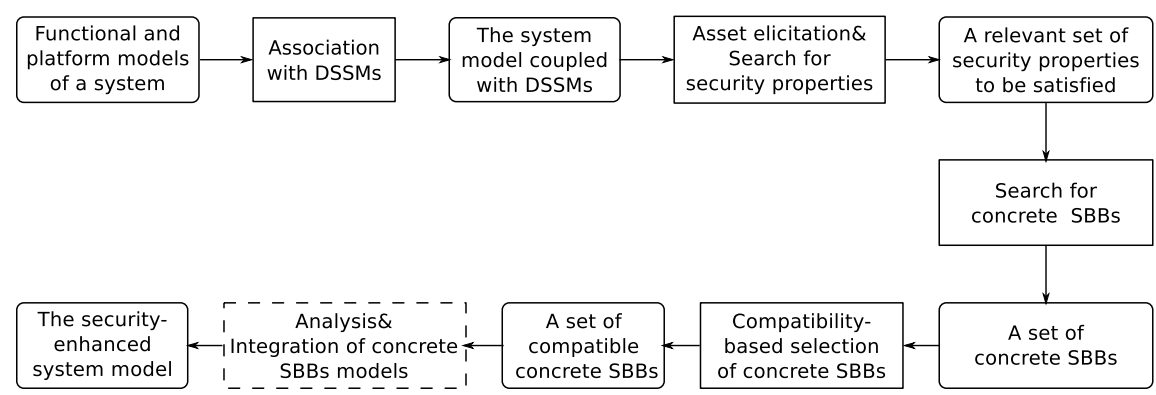

Figure 5.1: Application of the domain-specific security knowledge

The process starts from the functional and platform models of a system. In particular, we use SPACE models (see Section 5.1.1) to create a functional description of a system and UML class models annotated with some MARTE stereotypes (see Section 5.1.2) to create a platform description of a system. Thereafter, a suitable DSSM is selected and its elements are associated with the components of a functional model of a system (see Section 5.2). Note that if a required DSSM is not found, this step should be preceded by the DSSM creation step explained in the previous chapter. That is, the association step could be postponed until a suitable DSSM is created. 
The step Association with DSSMs is followed by the step Asset elicitation 6 Search for security properties (see Section 5.3) that results in a list of relevant security properties to be satisfied. Subsequently, concrete SBBs that satisfy the identified security properties are inferred from the enriched security ontology as indicated by the Search for concrete SBBs step. Thereafter, a related set of SPACE models are fetched, e.g. from the Arctis libraries. This step is explained in Section 5.4.

Due to the existence of different concrete SBBs, often various ways to secure an embedded system are possible that differ with regards to a range of criteria. For example, an engineer needs to ensure that a system under consideration will still perform the required functionality when security mechanisms are incorporated [42]. Additionally, when dealing with embedded systems, one needs to investigate how the added security mechanisms affect the consumption of crucial resources. The compliance of considered concrete SBBs to some standards can also affect a decision taken by a system engineer. A set of other possible criteria to be considered is proposed by Georg et al. [80]. Thus, to find a suitable solution one needs to carry out analysis of desired criteria and compare different alternatives. In our work, we introduced a so called model-based compatibility analysis technique applied at the Compatibility-based selection of concrete SBBs step. This analysis studies platform-related constraints of a system under development and alternative concrete SBBs.

Subsequently, a set of SPACE blocks that satisfy this criterion are integrated into a system model. At this point, other type of analysis are enabled, e.g. to verify that integration of concrete SBBs provides the required level of protection or that functional properties of a system are not violated by this increment. It is reflected by the Analysis\&Integration of concrete SBBs step. A dashed line of the box for this step in Figure 5.1 indicates that the elaboration of this step is not a contribution of this work. However, some types of analyses are in a focus of the research group that is developing the Arctis tool.

In the rest of this chapter, we explain each step outlined above using the case study introduced in Section 2 as a running example. We conclude this chapter with Section 5.6 that gives to a reader a broader view on the process of designing a security-enhanced embedded system. In particular, Section 5.6 extends the proposed process from Figure 5.1 highlighting additional steps and considerations that an embedded system engineer needs account for when integrating security mechanisms into a system.

\subsection{System Model}

In the following, we demonstrate the languages employed in our work for the functional and platform modelling. Section 5.1.1 illustrates a model of the measurements transfer scenario using the model-based engineering method SPACE. Section 5.1.2 shows an execution platform for a TSMC 
device defined as a MARTE model.

\subsubsection{Modelling a Functional Behaviour of a System}

To develop secure networked embedded systems, we employ the modelbased engineering method SPACE [3] described in Section 2.2. Recall that applications are composed of building blocks that can specify a local behaviour as well the interaction between several distributed entities. Similar to functional building blocks, security mechanisms can be expressed as self-contained building blocks. These SPACE building blocks that describe functionality of security mechanisms are concrete $S B B s$ introduced in Section 4.1.1. Note that as a result of applying the SPACE method (i.e. building a system as composition of reusable building blocks) the models used in different scenarios can share a lot of commonalities.

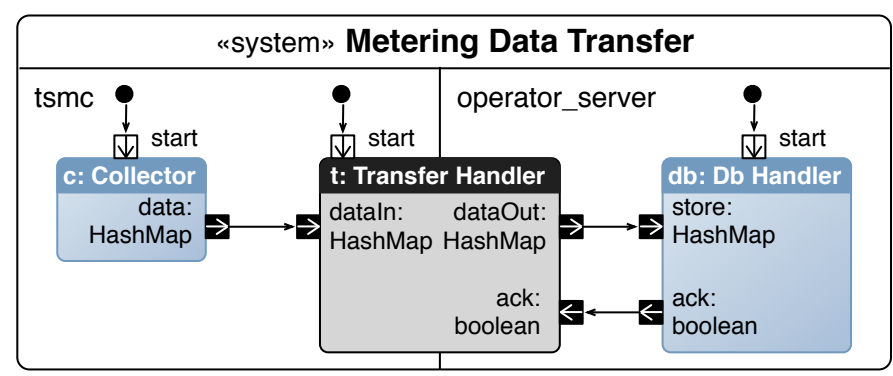

Figure 5.2: Functional system model of the measurement transfer scenario

Figure 5.2 depicts the measurement transfer scenario modelled in SPACE. It is a UML activity consisting of two partitions, namely tsmc and operator_server, that model the respective entities in our case study. The activity is composed of three building blocks that are connected with some "glue logic" through pins on their frames. The building block $c$ : Collector models periodic collection of measurement data from TSMs handled by a TSMC. The block $d b: D b$ Handler encapsulates the behaviour to store the data in a database of the operator. The block t: Transfer Handler manages the communication between the two components that, as will be described later, buffer the data, send it, and resend it in the case of a negative acknowledgement. The block $c$ and $d b$ are local blocks since they specify the local behaviour of an entity. In contrast, the block $t$ is a collaborative block as it also describes interaction between two entities. The three blocks $(c, d b$, and $t$ ), further, refer to activity diagrams that define their detailed internal behaviour as exemplified for the block $t$ in Figure 5.3.

The Petri net-like semantics of the activities models behaviour as control and object flows of tokens between the nodes of an activity via its edges. 
When a system starts, a token flows from each of the initial nodes $(\bullet)$ following the edges of the activity. In the application in Figure 5.2, all three inner blocks are started in the initial node. Then, periodically the collector block emits a token containing an object of type HashMap through its pin data. This object maps TSM identifiers to measurement values at a particular time. As depicted by the outgoing edge from pin data of block $c$, the object is forwarded to block $t$ and further to block $r$ : Reactive Buffer via its pin add (see Figure 5.3). This buffering block, which is taken from one of the Arctis libraries, is used to buffer measurement data that may arrive when other data is being sent but not yet acknowledged. If data is received when the buffer is empty, it is emitted immediately; otherwise, it is buffered. The pin next is used to get subsequent data. Following the outgoing edge of the pin out of the block $r$, a copy of measurement data is stored temporarily in variable temp by the operation set temp. Thereafter, the token flows through the merge node $(\diamond)$ and data is sent to the other entity as illustrated by the edge crossing the partition border. In the receiver partition, the data is forwarded out of the block which, according to Figure 5.2, is stored in a database by the block $d b$.

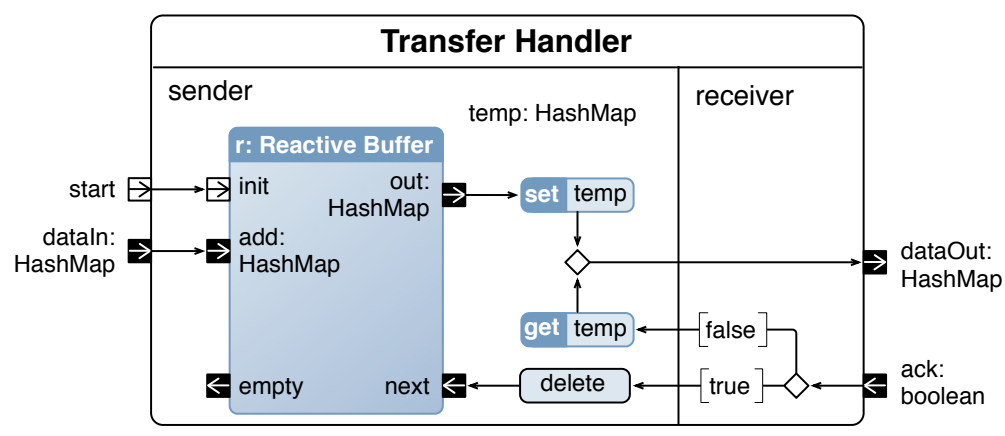

Figure 5.3: Detailed behaviour of the transfer handler block

The block db: Db Handler in Figure 5.2 will emit a token via the pin ack containing either a positive or a negative acknowledgement. A positive acknowledge corresponds to a successful transfer of measurement data, while a negative acknowledgement is issued in the case the received measurements have not passed the validation test executed by the $d b$ block. Thus, the token emitted via the pin ack flows further inside the block $t$ and, as depicted in Figure 5.3, reaches the decision node $(\diamond)$. A positive acknowledgement leads the token flows through the outgoing edge labelled with true. Thereafter, the operation delete, that removes the data stored in the variable temp, is called and subsequent data, if any, is retrieved from the buffer $r$. A negative acknowledgement moves the token through the edge labelled with false. In this case, the previously sent data is retrieved from the variable temp and 
sent again.

\subsubsection{Modelling an Execution Platform}

A platform model of a TSMC device for our scenario is depicted in Figure 5.4. We use UML class models annotated with stereotypes of the Hardware Resource Modelling (HRM) package of the MARTE profile (see Section 2.2). The modelling is done in the MagicDraw tool [9].

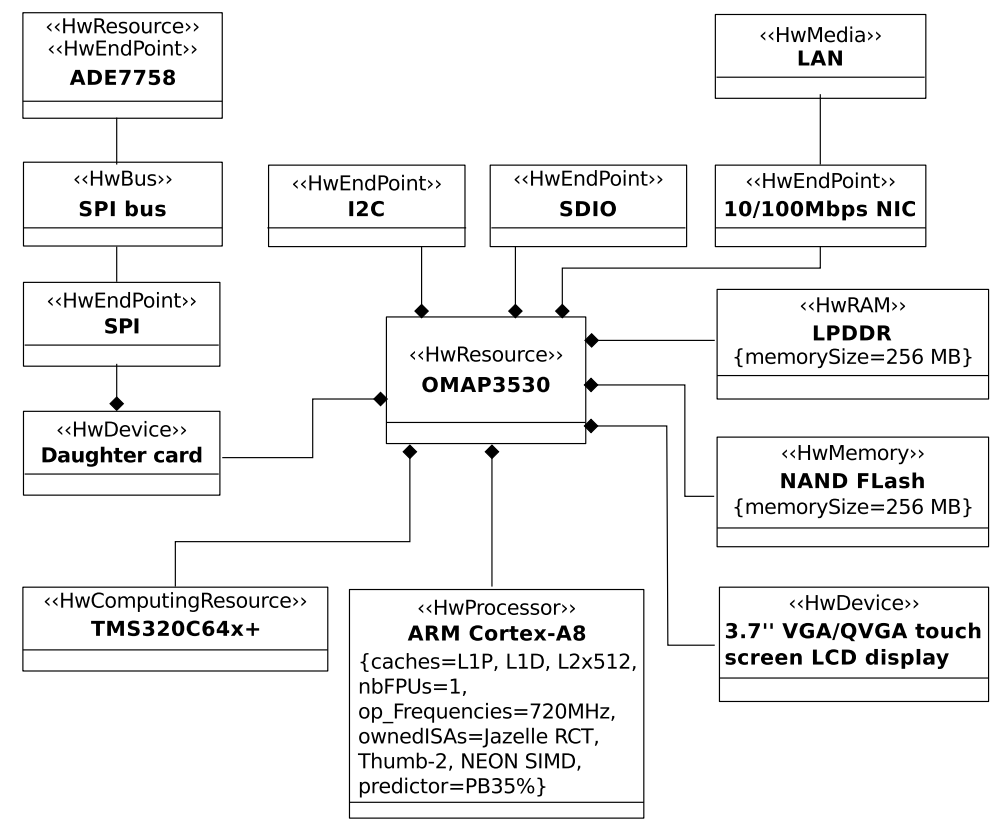

Figure 5.4: Platform model for a TSMC device

The main component of a TSMC platform is an OMPA3530 board [81]. This board includes computing elements (TMS320C64x+ DSP and ARM Cortex-A8), storage elements (NAND Flash and LPDDR), communication interfaces (I2C, SDIO, and 10/100Mbps NIC), a daughter card, and a LCD display. The daughter card is connected to the ADE7758 sensor via a Serial Peripheral Interface (SPI) bus. Finally, 10/100Mbps NIC is used to connect a TSMC to a communication channel (LAN). In the following, we briefly explain stereotypes used from the MARTE HRM package for modelling of a TSMC platform.

The HwResource is the most general term of the HRM that represents any hardware unit. The HwComputingResource denotes a computation unit, where the HwProcessor is its refinement that represents a processor or microcontroller unit. Similar, the HwMemory is an abstract memory unit that denotes a given amount of memory, where the $H w R A M$ refines it to repre- 
sent a unit of the random access memory. The HwMedia is a central term that represents a communication resource used to transfer data over some channel. In our example, we use the HwBus stereotype that models a wired channel. The HwEndPoint indicates that annotated components are connection points. Finally, the HwDevice stereotype refers to an entity that interfaces with an external environment.

\subsection{Association with DSSMs}

The goal of the step Association with DSSMs in the proposed process is twofold: (1) a DSSM that is relevant for a system under development is identified and selected; (2) bounds of a system (its functional model), where the knowledge captured by a selected DSSM should be applied, are established. Figure 5.5 depicts an interface of the SEED MagicDraw plug-in developed to support this step. We exemplify the Association with DSSMs step with our use case of the measurements transfer scenario.

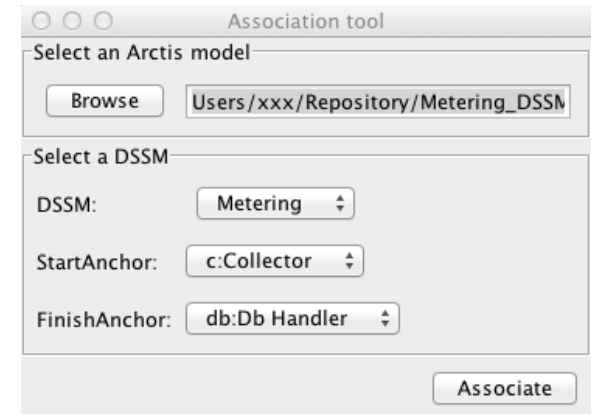

Figure 5.5: Association of the selected DSSM with the system elements (the user interface)

Since the case study is a smart metering application, an embedded system engineer selects the metering DSSM from the library of DSSMs (i.e. step (1)). Hence, the association is based on a matching of the system and security domains. Thereafter, those parts of a system containing data to be protected are identified (i.e. step (2)). In this thesis, we discuss the protection of the metering data that, in the functional system model from Figure 5.2, flows from the block $c$ : Collector in the TSMC to the $d b: D b$ Handler in the operator server. Thus, these two blocks are the starting respective end points of the object flow to be protected. Therefore, the identifiers of these two blocks are assigned to the fields StartAnchor and FinishAnchor respectively as it is shown in Figure 5.5. 


\subsection{Asset Elicitation and Search for Security Properties}

In this section, we present the asset elicitation technique along with the search for security properties method. The developed asset elicitation technique consists of two steps. The first step inspects a functional model identifying present assets. This step uses a set of rules elaborated for traversing a functional model and the information about assets relevant for a certain domain obtained from an associated DSSM. Thereafter, we retrieve from the enriched security ontology a set of security properties associated with the identified assets through the DSSM. Further, we proceed with the second step of the asset elicitation technique. This step utilises a platform model of a system and information about their potential threats to identify vulnerable assets. The output, when the asset elicitation technique is applied, is a set of assets and security properties that associate security goals and defence strategies with the identified set of assets.

In Section 5.3.1, we define a set of rules that allow identifying assets to be protected within a functional system model. Section 5.3.2 shows a method to retrieve a set of security properties relevant for the identified assets. Then, we explain the developed approach to refine a set of identified assets and corresponding security properties utilising the platform description information. This method is presented in Section 5.3.3.

\subsubsection{Asset Elicitation on a Functional Model}

The first step of the proposed asset elicitation technique is to identify an initial set of security assets utilising a functional model of a system. This identification is implemented as rule-based classification of assets developed in this work. Thus, the rule-based classification is a method that allows identifying assets within a functional model of a system and their matching to the classes defined in the core security ontology introduced in Section 4.1.1. In particular, the considered classes are "data stationary" and "data in transit".

The rule-based classification is realised as application of the rules $\mathbf{R} \mathbf{1}$ - R7 to collaborative-based Arctis models. These rules are presented in Figure 5.6. We have implemented this functionality in a tool called asset analyser. Afterwards, an engineer complements this classification according to an associated DSSM. However, it is worth noting that the latter task can potentially be automated given a system modelling language closely tailored to a domain (i.e. a domain-specific language). We now proceed to explain our rules (see Figure 5.6) and their application logic (see Figure 5.8).

According to the SPACE semantics [39], an activity is a directed graph $g$ with a set of activity nodes $V$ and connecting edges $E$, i.e $g=(V, E)$. Figure 5.6 presents the seven identification rules $\mathbf{R} 1$ to R7. In the rules, we use the following functions: 
- Two functions mapping an activity node and edge to their particular types, i.e. $\operatorname{kind}_{V}: V \rightarrow K_{V}$ and kind $_{E}: E \rightarrow K_{E}$, where $K_{V}=$ \{operation, merge, join, fork, decision, local, collaboration, other\} and $K_{E}=\{$ object, control $\}$.

- The set $O N$ of all object nodes of a given activity, i.e. the data stored in the system and transported within the data flow tokens.

- Two functions mapping a given node to the set of its incoming and outgoing edges, i.e. $i n_{E}: V \rightarrow 2^{E}$ and out $E: V \rightarrow 2^{E}$.

- Two functions returning an object flowing to (reps. from) a given node through an edge, i.e. $i n_{O}: E \times V \rightarrow O N$ and out $_{O}: V \times E \rightarrow O N$.

- A function mapping a node to a set of partitions to which it belongs, i.e. part: $V \rightarrow 2^{P}$, where $P$ is a set of all partitions of a given activity diagram.

- Two functions that return the source and target nodes of a given edge, i.e. source $: E \rightarrow V$ and target $: E \rightarrow V$ respectively.

- A function mapping a merge node and the set of its incoming object edges to its outgoing object, i.e. $f$ Merge $: V \times 2^{E} \rightarrow O N$. Likewise, we define function $f$ Join for a join node. According to the SPACE semantics, only one outgoing edge is allowed for merge and join nodes (see the rule OUT1 in [39]).

- A function mapping a fork node, its incoming object edge, and one of its outgoing edges to an object flowing through this outgoing edge, i.e. fFork : $V \times 2^{E} \times E \rightarrow O N$. Likewise, we define function $f$ Decision for a decision node. For the sake of generality, we allow that the second argument of fFork and fDecision is a set of edges. However, according to the SPACE semantics [39], fork and decision nodes can have only a single incoming edge (see the rule IN3 in [39]).

- A function mapping a given asset to a class from the core security ontology, i.e. class : $A \rightarrow K_{A}$, where $K_{A}=\{$ transit, stationary $\}$ and $A$ is the set of assets constructed from elements of the set $O N$. In particular, each asset is uniquely identified as a tuple $\langle O N, V, E\rangle$.

- A function that takes a certain activity (i.e. a graph) $g \in G$ and returns a set of assets elicited within this activity $g$, i.e. $f a: G \rightarrow 2^{A}$, where $G$ is a set of activities. 
$q \in V, e \in \operatorname{in}_{E}(q), \operatorname{kind}_{E}(e)=$ object,

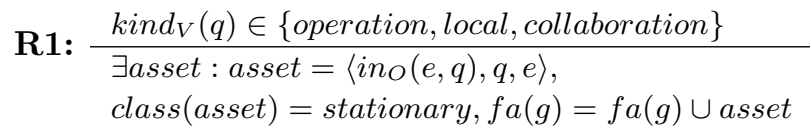

$q \in V, e \in \operatorname{out}_{E}(q), \operatorname{kind}_{E}(e)=$ object,

R2: $\frac{\operatorname{kind}_{V}(q) \in\{\text { operation, local, collaboration }\}}{\exists \text { asset }: \text { asset }=\left\langle\text { out }_{O}(q, e), q, e\right\rangle,}$

class $($ asset $)=$ stationary, $f a(g)=f a(g) \cup$ asset

$e \in E, \operatorname{kind}_{E}(e)=$ object, $|\operatorname{part}(\operatorname{source}(e))|=|\operatorname{part}(\operatorname{target}(e))|=1$, $\operatorname{part}(\operatorname{source}(e)) \cap \operatorname{part}(\operatorname{target}(e))=\emptyset, q \in V$,

R3: $\operatorname{kind}_{V}(q) \in\{$ operation, local $\}, e \in$ out $_{E}(q)$

$\exists$ asset $:$ asset $=\left\langle\right.$ out $\left._{O}(q, e), q, e\right\rangle$,

class $($ asset $)=$ transit, $f a(g)=f a(g) \cup$ asset

$e \in E, \operatorname{kind}_{E}(e)=$ object,$|\operatorname{part}(\operatorname{source}(e))|=|\operatorname{part}(\operatorname{target}(e))|=1$,

$\operatorname{part}(\operatorname{source}(e)) \cap \operatorname{part}(\operatorname{target}(e))=\emptyset, m \in V, \operatorname{kind}_{V}(m)=m e r g e$,

R4: $\quad e \in$ out $_{E}(m), q \in V$, in $_{E}(m) \cap$ out $_{E}(q) \neq \emptyset, \operatorname{kind}_{V}(q) \in K_{V} \backslash\{$ other $\}$

$\exists$ asset $:$ asset $=\left\langle f \operatorname{Merge}\left(m, \operatorname{in}_{E}(m)\right), m, e\right\rangle$,

class $($ asset $)=$ transit, $f a(g)=f a(g) \cup$ asset

$e \in E, \operatorname{kind}_{E}(e)=$ object, $|\operatorname{part}(\operatorname{source}(e))|=|\operatorname{part}(\operatorname{target}(e))|=1$, $\operatorname{part}(\operatorname{source}(e)) \cap \operatorname{part}(\operatorname{target}(e))=\emptyset, d \in V, \operatorname{kind}_{V}(d)=\operatorname{decision}$,

R5: $\quad e \in$ out $_{E}(d), q \in V$, in $_{E}(d) \cap$ out $_{E}(q) \neq \emptyset, \operatorname{kind}_{V}(q) \in K_{V} \backslash\{$ other $\}$

$\exists$ asset $:$ asset $=\left\langle f\right.$ Decision $\left.\left(d, i_{E}(d), e\right), d, e\right\rangle$,

class $($ asset $)=$ transit, $f a(g)=f a(g) \cup$ asset

$e \in E, \operatorname{kind}_{E}(e)=$ object, $|\operatorname{part}(\operatorname{source}(e))|=|\operatorname{part}(\operatorname{target}(e))|=1$,

$\operatorname{part}(\operatorname{source}(e)) \cap \operatorname{part}(\operatorname{target}(e))=\emptyset, j \in V, \operatorname{kind}_{V}(j)=j$ oin,

R6: $\quad e \in \operatorname{out}_{E}(j), q \in V, \operatorname{in}_{E}(j) \cap$ out $_{E}(q) \neq \emptyset, \operatorname{kind}_{V}(q) \in K_{V} \backslash\{$ other $\}$

$\exists$ assetasset $=\left\langle f \operatorname{Join}\left(j, i_{E}(j)\right), j, e\right\rangle$,

class $($ asset $)=$ transit, $f a(g)=f a(g) \cup$ asset

$e \in E, \operatorname{kind}_{E}(e)=$ object, $|\operatorname{part}(\operatorname{source}(e))|=|\operatorname{part}(\operatorname{target}(e))|=1$, $\operatorname{part}(\operatorname{source}(e)) \cap \operatorname{part}(\operatorname{target}(e))=\emptyset, f \in V, \operatorname{kind}_{V}(f)=$ fork,

R7: $\quad e \in$ out $_{E}(f), q \in V$, in $_{E}(f) \cap$ out $_{E}(q) \neq \emptyset, \operatorname{kind}_{V}(q) \in K_{V} \backslash\{$ other $\}$

$\exists$ asset $:$ asset $=\left\langle f\right.$ Fork $\left.\left(f, i n_{E}(f), e\right), f, e\right\rangle$,

class $($ asset $)=$ transit, $f a(g)=f a(g) \cup$ asset

Figure 5.6: Rules of the asset identification technique

The asset elicitation rules defined in Figure 5.6 form a set of assets for an activity $g$ that is accessed through $f a(g)$. Thus, each time a rule 
identifies an asset, i.e. asset, this asset is added into a set of elicited assets for $g$, i.e. $f a(g)=f a(g) \cup$ asset. We require that initially (i.e. before the elicitation process starts) the set of assets for an activity $g$ is an empty set, i.e. $f a(g)=\emptyset$. We continue describing in more detail each rule from Figure 5.6 and their application logic.

The rules $\mathbf{R} 1$ and $\mathbf{R 2}$ express that for an operation, local, or collaboration node $q$ a stationary data asset (i.e. asset) is observed if this node has an incoming (R1) resp. outgoing (R2) edge $e$ of the kind object. The rules $\mathbf{R 3}$ to $\mathbf{R 7}$ are applied to an object flow crossing a border of two partitions, which corresponds to the data in transit concept. R3 describes the case that an object leaves an operation node and goes directly to another partition. By the rules $\mathbf{R} 4$ to $\mathbf{R 7}$, we cover the cases that a flow passes a merge, join, decision, or fork node before crossing a partition border. Figures. 5.7.(a) to 5.7. (e) illustrate the cases of $\mathbf{R} \mathbf{3}$ to $\mathbf{R} 7$ respectively.

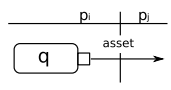

(a) Simple case (R3)

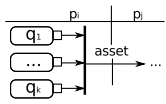

(d) Join case $(\mathbf{R 6})$

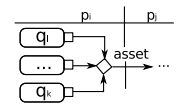

(b)

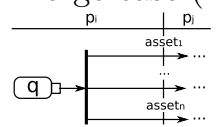

(e) Fork case $(\mathbf{R} 7)$

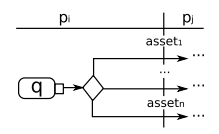

(c) Decision case (R5)

Figure 5.7: Illustration of the rules

The application of the rules $\mathbf{R} \mathbf{1}-\mathbf{R} 7$ to a functional system model is outlined by the traverseBlocks and traverseEdges functions depicted in Figure 5.8. Recall that each activity (i.e. graph) $g$ is represented by a pair of nodes and edges $(V, E)$. First, the function traverseBlocks traverses all nodes $V$ and applies the rules $\mathbf{R} \mathbf{1}$ and $\mathbf{R 2}$. For example, an application of this function to the model in Figure 5.2 will identify six data stationary assets, which are data, dataIn, dataOut, store, and two acks.

Thereafter, if a considered node is a local block, the traverseBlocks function is recursively applied to its internal behaviour. Likewise, a collaborative block invokes both the traverseBlocks and traverseEdges functions. For example, this is the case for the analysis of the $t$ block in Figure 5.3. Here, the function traverseEdges applies the $\mathbf{R} \mathbf{4}$ rule to the merge node before the crossing edge from partition sender to receiver. For the decision node before the crossing edges in the opposite direction, the rule R5 is used. As a result, four data in transit assets are elicited: two ack assets incoming to the get and delete nodes; two temp assets outgoing from the get and set nodes.

Table 5.1 summarises the results of applying the rules to our measurement transfer scenario described in Figure 5.2. For those assets that have 


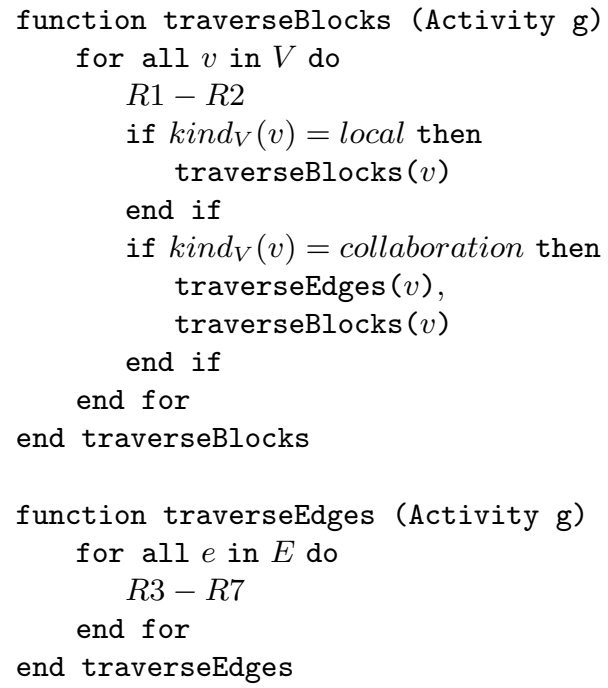

Figure 5.8: Functions to traverse a functional system model

duplicating names (i.e. ack and temp), we have added their location information in brackets.

Table 5.1: Results of eliciting assets from the functional model

\begin{tabular}{l|ll}
\hline Asset & DSSM classification & \\
\hline $\begin{array}{l}\text { data, dataIn, dataOut, store, out, } \\
\text { add, two temp (to and from the set } \\
\text { operation), temp (from the get op- } \\
\text { eration), }\end{array}$ & $\begin{array}{l}\text { StoredMeasurement (Data Sta- } \\
\text { tionary) }\end{array}$ & \\
\hline $\begin{array}{l}\text { ack (from the } d b \text { block), ack (to } \\
\text { the } t \text { blocks) }\end{array}$ & Not an asset (Data Stationary) \\
\hline temp (from the merge node) & CollectorToServerMsr (Data in \\
& Transit) & \\
\hline $\begin{array}{l}\text { ack (that goes from the decision } \\
\text { node to the operations get), ack } \\
\text { (that goes from the decision node } \\
\text { to the operations delete) }\end{array}$ & Not an asset (Data in Transit) \\
\hline
\end{tabular}

Figure 5.9 demonstrates the interface of the developed asset analyser tool when it is applied to the scenario of the MixedMode use case from the Figure 5.2. The main output of this tool is a table where each row represents an identified asset. First four columns contain information elicited by the algorithm (the functions and rules) presented in Figure 5.7, namely the name of an asset, its source (the NodeId | EdgeId column), and its class according to the core security ontology (the Ontology classification 
column). Classes in the column DSSM classification are assigned by an engineer among possible alternative classes captured in an associated DSSM. That is the metering DSSM for this example where the alternative classes are StoredMeasurement for data stationary assets, and CollectorToServerMsr and SensorToMeterMsr for data in transit assets (see Figure 4.4).

\begin{tabular}{|c|c|c|c|}
\hline \multicolumn{2}{|l|}{000} & analyser & \\
\hline \multicolumn{4}{|l|}{ Info } \\
\hline The selected model: & \multicolumn{3}{|c|}{ MeteringSystem.uml } \\
\hline The selected DSM: & \multicolumn{3}{|l|}{ Metering } \\
\hline \multicolumn{4}{|l|}{ Elicitation results } \\
\hline Asset name & Nodeld | Edgeld & \multicolumn{2}{|c|}{ Ontology classific... DSM classification } \\
\hline data & c 1 e2 & Data stationary & StoredMeasurem... \\
\hline dataln & $\mathrm{t} \mid \mathrm{e} 2$ & Data stationary & StoredMeasurem... \\
\hline dataOut & $\mathrm{t} \mid \mathrm{e} 4$ & Data stationary & StoredMeasurem... \\
\hline store & $\mathrm{db} \mid \mathrm{e} 4$ & Data stationary & StoredMeasurem... \\
\hline add & $\mathrm{r} \mid \mathrm{e} 4$ & Data stationary & StoredMeasurem... \\
\hline temp & set | el & Data stationary & StoredMeasurem... \\
\hline temp & set | e2 & Data stationary & StoredMeasurem... \\
\hline temp & get | e 10 & Data stationary & StoredMeasurem... \\
\hline ack & $\mathrm{db} \mid \mathrm{e} 5$ & Data stationary & not an asset \\
\hline ack & $\mathrm{t} \mid \mathrm{e} 5$ & Data stationary & not an asset \\
\hline temp & $\mathrm{m} 1 \mid \mathrm{e} 3$ & Data in transit & CollectorToServer... \\
\hline \multirow[t]{2}{*}{ ack } & d $1 \mid$ e 6 & Data in transit & not an asset \\
\hline & & & arch Concrete SBBs \\
\hline
\end{tabular}

Figure 5.9: Asset analyser tool (the user interface)

\subsubsection{Search for Security Properties}

Once the classification of the elicited assets is known a set of corresponding security properties can be retrieved from the enriched security ontology. Recall that the security property concept is defined in the core security ontology as the triple of asset, security goal, and defence strategy. These triples are generated when a DSSM (i.e. an object diagram) is transformed into a set of axioms that are added into the enriched security ontology. Thus, security properties which are available for a given asset can be accessed in the enriched security ontology executing the following query:

\section{SecurityProperty and has value [Asset]}

This query is formulated as an expression written in the Manchester syntax [82]. The Manchester syntax uses the standard description logic notation to specify restrictions (e.g. $\exists, \forall, \in$ ) replacing them with the English 
language keywords (e.g. some, only, value respectively). All boolean constructs (i.e. $\sqcap, \sqcup, \neg$ ) are also replaced by the English language keywords (i.e. and, or, not respectively). The rest of the words in the query defined above are names of the corresponding concepts and relations from the core security ontology depicted in Figure 4.2. The values in square brackets denote parameters of the query. We employ the HermiT reasoner [83] to execute this query.

For example, for those assets that are classified as CollectorToServerMsr and StoredMeasurement (see Table 5.1) the following set of security properties is retrieved:

[CollectorToServerMsr, Confidentiality, Prevention]

[CollectorToServerMsr, Integrity, Detection]

[CollectorToServerMsr, Authentication, Detection]

[StoredMeasurement, Confidentiality, Prevention]

[StoredMeasurement, Integrity, Prevention]

\subsubsection{Asset Elicitation Utilising a Platform Model}

In this section, we explain the second step of the asset elicitation technique. In particular, we present a method that refines the results of the rule-based classification (introduced in Section 5.3.1) by utilising a platform model of a system.

This method is applied when the initial set of assets and related security properties are identified inspecting a functional model. Three steps that compose this method are shown in Figure 5.10. These steps allow identifying which security properties must be considered as those security properties to be satisfied given a platform model. Thus, this extending method allows focusing on a set of relevant assets refining the results from the previous steps (see Sections 5.3.1 and 5.3.2). We use the platform model selected for a TSMC block presented in Section 5.1.2.

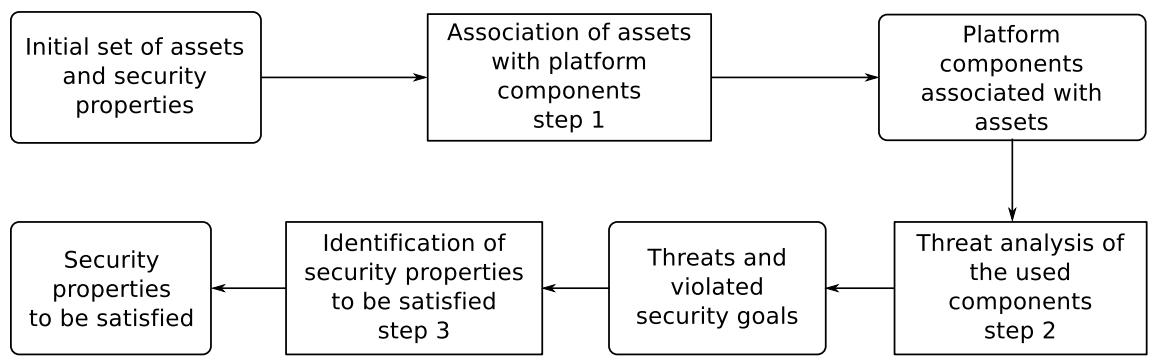

Figure 5.10: Asset elicitation technique utilising a platform model

At the step 1, initially elicited assets are associated with available platform resources, e.g. communication, computing, and storage components. 
In general, any platform components that are involved in operations with assets should be mentioned during this association. In particular, we provide the following basic guideline:

1. Associate each data stationary asset with a computing unit that operates on it (i.e. components annotated with the HwProcessor stereotype and its subclasses) and a memory unit that stores it (i.e. components annotated with the HwMemory stereotype and its subclasses).

2. Associate each data in transit asset with a communication channel that is used to transmit this asset (i.e. components annotated with the HwMedia stereotype and its subclasses) and with two interfaces on the sender and receiver ends (i.e. the HwEndPoint stereotype).

3. Associate each data stationary asset with some resource (i.e. components annotated with the HwResource or HwDevice stereotypes) that contains computing and memory units, which operate with the asset and store it respectively.

4. Associate each data in transit asset with some resource (i.e. components annotated with the HwResource or HwDevice stereotypes) that contains sender and receiver interfaces and a communication channel, which are involved in transmission of the asset.

Table 5.2 demonstrates association of the assets elicited in Section 5.3.1 with the components of the TSMC platform depicted in Figure 5.4. The data asset is associated with the ADE7758 component (the third rule of our guideline). All other data stationary assets (row 2 and 3 in Table 5.2) are associated with the NAND Flash or LPDDR components (i.e. memory units) and the ARM Cortex-A8 component (i.e. computing unit) as it is instructed by the first rule of the guideline. Finally, following the second rule, the data in transit assets (row 4 in Table 5.2) are associated with the 10/100Mbsp NIC components of the TSMC device and of the operator server host (not shown in Figure 5.4) and onto the LAN, which is used as a communication channel.

Step 2 from Figure 5.10 involves analysis of existing (known) threats for components of the used execution platform. In general, this task may imply collaboration between a security expert and a system engineer, but the use of threat repositories can facilitate this task. For example, an engineer can query an ontology like one presented by Herzog et al. [67] potentially extended with other expert knowledge about existing threats. In our case, we use knowledge acquired within the SecFutur project employing the CORAS method [84], combined with the results of the threat analysis for embedded system platforms published by Ravi et al. [85]. Table 5.3 shows the identified threats and potentially violated security goals.

The last step of the asset elicitation technique (i.e. step 3 in Figure 5.10), automatically identifies a set of security properties to be satisfied. The 
Table 5.2: Association of the assets with the platform components

\begin{tabular}{|c|c|c|}
\hline Asset & Classification & Association \\
\hline data & StoredMeasurement & ADE7758 \\
\hline $\begin{array}{l}\text { dataIn, dataOut, } \\
\text { out, add }\end{array}$ & StoredMeasurement & $\begin{array}{l}\text { [NAND Flash, ARM } \\
\text { Cortex-A8] }\end{array}$ \\
\hline $\begin{array}{l}\text { two temp (to and } \\
\text { from the set oper- } \\
\text { ation), temp (from } \\
\text { the get operation) }\end{array}$ & StoredMeasurement & $\begin{array}{l}{[\text { LPDDR, }} \\
\text { Cortex-A8] }\end{array}$ \\
\hline $\begin{array}{l}\text { temp (from the } \\
\text { merger node) }\end{array}$ & CollectorToServerMsr & $\begin{array}{l}\text { [OMAP3530: } \\
\text { 10/100Mbsp NIC, } \\
\text { LAN, } \quad \text { DBHost: } \\
\text { 10/100Mbsp NIC] }\end{array}$ \\
\hline
\end{tabular}

Table 5.3: Threats and violated security goals

\begin{tabular}{l|l|l}
\hline $\begin{array}{l}\text { Platform compo- } \\
\text { nent }\end{array}$ & Threat & $\begin{array}{l}\text { Violated security } \\
\text { goal }\end{array}$ \\
\hline NAND Flash & injection & integrity \\
\hline LAN & eavesdropping & confidentiality \\
\hline
\end{tabular}

identification algorithm is implemented as follows. The security goal of each earlier retrieved security property (explained in Section 5.3.1) is compared to the security goal violated by the threat (see Table 5.3), which targets a platform component associated with an asset of the considered security property (see Table 5.2). Now, if the security goal of the security property is equal to the security goal violated by the threat, then this security property is added to the set of security properties to be satisfied. In our scenario, we have extracted the following set of security properties to be satisfied:

- $S P_{1}$ : [StoredMeasurement, Integrity, Prevention]

- $\mathrm{SP}_{2}$ : [CollectorToServerMsr, Confidentiality, Prevention]

$S P_{1}$ is formulated due to the knowledge about the existence of an injection threat that violates integrity of the NAND Flash component (see Table 5.3) used in association of the StoredMeasurement asset (see Table 5.2). Similarly, $S P_{2}$ is identified due to the presence of an eavesdropping threat that violates confidentiality of the LAN component (see Table 5.3), which is used in association of the CollectorToServerMsr asset (see Table $5.2)$. 


\subsection{Search for Concrete SBBs}

At this step, a set of identified security properties to be satisfied are used to find a set of concrete SBBs. They are, for example, the $S P_{1}$ and $S P_{2}$ security properties for the metering scenario. Concrete SBBs for a particular domain and security properties described within an associated DSSM are retrieved from the enriched security ontology executing the following query ${ }^{1}$ :

\section{ConcreteSBB and (satisfies value [SecurityProperty]) and implements some (AbstractSBB and belongsTo value [Domain])}

Execution of the above query for $S P_{1}$ and $S P_{2}$ retrieves two concrete SBBs for the StoredMeasurement asset, namely SecFutur secure storage and SecFutur TPM seal ${ }^{2}$ and two concrete SBBs for the CollectorToServerMsr asset, namely $A E S$ and $D E S$.

Due to the existence of several alternatives to secure a considered scenario an engineer needs to carry out an additional analysis. This is the case of the measurement transfer scenario above. For example, this analysis may include investigation of the resource overhead introduced by concrete SBBs. Our work contributes to one kind of such analyses proposing a technique that inspects and matches platform constraints of candidate concrete SBBs and constraints of an execution platform model adopted for a design of an embedded system under development. Recall that platform constraints for concrete SBBs are obtained as results of performance evaluation of these security solutions and captured by corresponding PERs that are consequently stored in the enriched evaluation ontology. This step is reflected in Figure 5.1 by the Compatibility-based selection of concrete $S B B s$ and presented in the following sections. Different types of (trade-off) analyses are also enabled by our contributions since each PER can be used to store other data about results of performance evaluation of a concrete SBB.

Other possible criteria for selection of concrete SBBs could be, for example, their effect on the original functionality of a system and the cost of the concrete SBBs' integration. Formalisation of these needs goes beyond the contributions of this thesis. However, our process accounts this necessity as the Analysis\&Integration of concrete SBBs step in Figure 5.1.

For the illustration purposes, let us assume that a system engineer decides to use the AES concrete $\mathrm{SBB}$ to satisfy $S P_{2}$. As a result, the system engineer is directed towards a pair of Arctis blocks, namely AES Encryption and AES Decryption.

As mentioned in Section 4.1.1, integrating a concrete SBB may create new assets as expressed by the creates and requires relations in the core security ontology. Hence, a further search of concrete SBBs, i.e. a recursive application of the above mentioned query, is needed to fulfil the security

\footnotetext{
${ }^{1}$ The query is written in the Manchester syntax [82]

${ }^{2}$ The concrete SBBs that start with the suffix "SecFutur" are developed within the SecFutur [10] European project.
} 
goals required for these new assets. For this query, the security property is composed of the created asset and its required security goal, namely the requiredGoal attribute in Figure 4.3. The search is done within the domain specified by the externalDSSM attribute in Figure 4.3. As a result, search of concrete SBBs will continue until all security goals of all created assets are fulfilled. Alternatively, this search will lead to an empty set of SBBs indicating that a vulnerability remains in terms of an unprotected asset. In other words, the search for concrete SBBs results in building alternative sets of concrete SBBs. Each set satisfies a considered security property.

Note that such an approach can lead to a cycle since an ontology reasoner exhaustively searches for any concrete SBB in a DSSM that satisfies the security property. For example, the query can return the same concrete SBB that has invoked it if this concrete SBB satisfies the same security property required by its created asset. To handle such occurrences, we have developed an algorithm that detects and resolves such cycle conditions.

This algorithm is based on constructing a directed graph while the search for concrete SBBs goes on:

- Create a node for each found concrete SBB and asset.

- Create a directed edge from a concrete SBB to an asset if the concrete SBB creates the asset.

- Create a directed edge from an asset to a concrete SBB if the concrete SBB protects the asset.

Then, we use a cycle detection algorithm (one based on identification of backward edges during execution the DFS (Depth-first search) algorithm [86]) to detect cycles in the constructed graph. The search continues if there are still alternative paths ignoring (by removing) the detected cycles. Otherwise, the engineer is notified of the remaining unprotected asset.

A nice property of the previously selected Arctis blocks (i.e. the AES pair) is that they already contain a protection of the keys, i.e. new assets are not created. Thus, we can directly continue with the integration of these blocks. The integration of the AES blocks (encryption and decryption) is easily done by arranging their instances before and after the block t: Transfer Handler as shown in Figure 5.11.

The functionality described above is implemented as a tool called concrete $S B B$ searcher. The user interface of this tool is depicted in Figure 5.12. In this tool, the list "Security properties" contains a list of relevant security properties for a selected asset (e.g. CollectorToServerMsr). These properties are represented in a form of tuples, i.e. [asset, security goal, defence strategy].

The bottom part of the GUI screen shot in Figure 5.12 contains a tree representation of found sets of concrete SBBs. The root item denotes the selected for the search security property. For this example, we use the earlier retrieved security property $S_{2}$ that is [CollectorToServerMsr, 


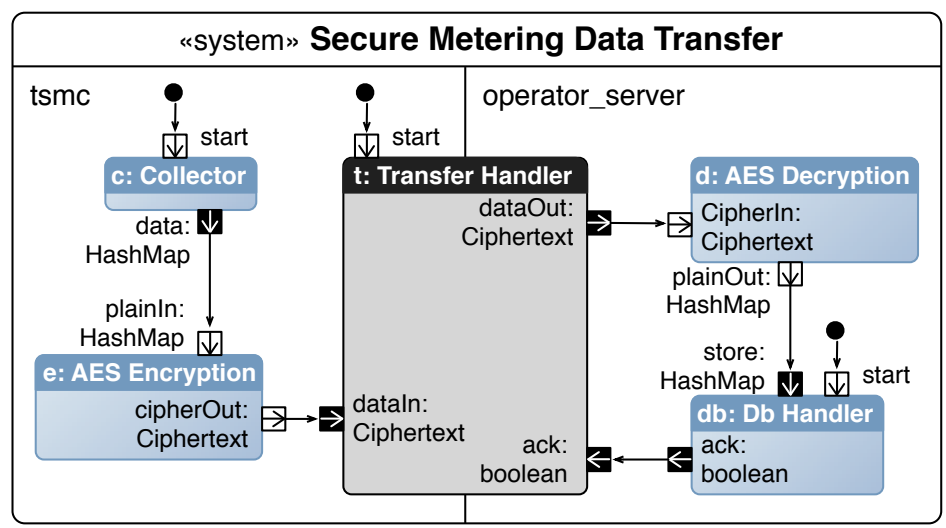

Figure 5.11: Adapted model protecting the transfer of measurement data

Confidentiality, Prevention]. The root item expands to several items that have the following format:

\section{[asset] requires [security goal]: [concrete SBB]}

In this string, [asset] and [security goal] are elements from the selected for the search security property, i.e. CollectorToServerMsr and Confidentiality respectively from $\mathrm{SP}_{2}$. Then, [concrete SBB] denotes a retrieved concrete SBB that satisfies this security property. In our example, they are OpenSSL, DES, and AES implementations. Note that the AES SBB has a special icon, i.e. "CA" (Created Assets). This icon denotes that the AES SBB, in turn, creates some assets to be protected. An engineer needs to expand this item, to see the created assets, their required security goals, and available concrete SBBs. Thus, a system engineer can see all possible alternative sets of concrete SBBs that together can be used to protect a considered asset.

\subsection{Compatibility-based Selection of Concrete SBBs}

The last step that we describe in this thesis is the Compatibility-based selection of concrete $S B B s^{3}$. It is supported by the method for model-based compatibility analysis developed in this work. We begin with introducing a reader into the context in Section 5.5.1. In particular, we recall some

\footnotetext{
${ }^{3}$ In this section, the term SBB can be understood as the term RBB used in Section 4.1.2
} 


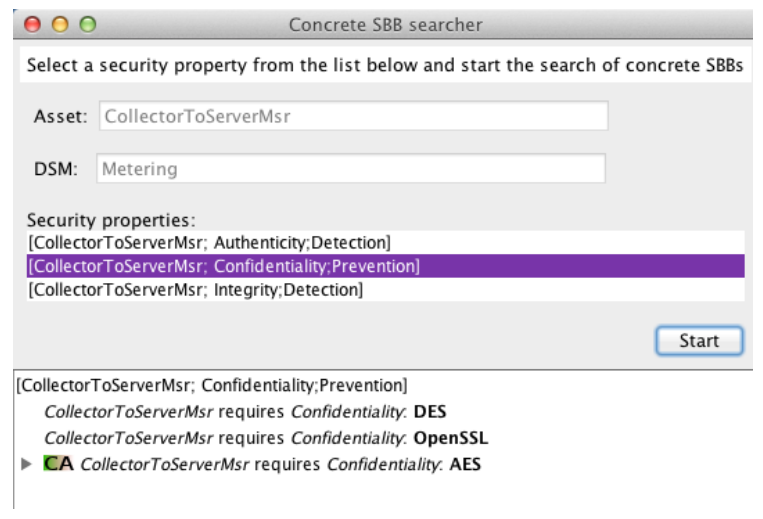

Figure 5.12: Concrete SBB searcher tool (the user interface)

concepts introduced earlier in this thesis and outline basics of the proposed method. Thereafter, Sections 5.5.2 - 5.5.4 explain the method for modelbased compatibility analysis.

\subsubsection{Introduction into the Compatibility Analysis}

Adding a new feature to a system always comes with resource claims. In Section 4.1.2, we introduced the PER (Performance Evaluation Record) concepts that enables capturing results of performance analysis of SBBs conducted by domain security experts. Among other information, PERs store data about resource footprint and quality of extra-functional properties provided by concrete SBBs. Thus, an embedded system engineer can use this information already at the early design phase increasing the efficiency of an embedded system design process. Hence, the PER concepts contributes to selection of a suitable set of concrete SBBs by enabling the sensitivity and trade-off analyses at early phases.

Another useful and significant input to the design process is knowledge about platform-specific constraints of concrete SBBs. These constraints originate from the fact that SBBs (and just RBBs) for embedded systems are often optimised to exploit a particular feature of hardware components on which they are implemented. Thus, they can provide good quality of extra-functional properties (i.e. level of security) while consuming a small amount of limited resources. In our studies, we argue that these constraints also need to be documented and accounted in order to support integration of concrete SBBs into embedded systems. For example, Preissig [76] reports the results of performance analysis of the Data Encryption Standard (DES) implementation optimised for the memory architecture of the used chip. Similarly, other implementations of DES rely on the presence of a certain instruction set to accelerate permutation operations [6]. Therefore, this information is included as a part of a PER using the gemRequiredComponent 
stereotype from the GEM profile explained in Section 4.1.2.

Consequently, each PER is transformed into an ontology called enriched evaluation ontology that allows storing and structuring this field knowledge. As a result, a variety of information can be retrieved from this ontology defining appropriate queries for the ontology, for example:

- Retrieve values of relevant performance metrics for a certain SBB.

- Retrieve a set of SBBs of a particular domain that satisfy required values w.r.t. certain performance metrics.

- Retrieve a set of SBBs, for which the platform constraints are compatible with a platform adopted for an embedded system under development.

Queries like the first two can be directly implemented as SPARQL queries [60]. However, the task of compatibility analysis, i.e. the third query in the list above, requires more sophisticated support. Consequently, we develop such a support as a method that implements model-based compatibility analysis.

This analysis allows automatically accounting for platform constraints while selecting a set of SBBs to be used for a system design. An extrafunctional domain expert (in our study, a security expert) provides these constraints by annotating elements of an evaluation platform (i.e. a MARTE model) with the gemRequiredComponent stereotype (see Figure 4.8).

The core of our method is a set of ontologies and SPARQL queries designed to infer whether concrete SBBs and an adopted platform for the embedded system are compatible (i.e. whether the formulated platform constraints for a concrete SBB fits platform declarations of the system being configured). The SEED MagicDraw plug-in implements this functionality to support the use of the compatibility analysis.

In the following, Section 5.5.2 explains the developed hierarchy of ontologies and Section 5.5.3 defines the notion of compatibility. Section 5.5.4 shows results of scalability and performance estimations for our approach.

\subsubsection{Ontologies for Compatibility Analysis}

Figure 5.13 depicts the developed set of ontologies. These ontologies are organised in three layers: expert, vendor, and engineer layers. The name of each layer denotes its main actor. These ontologies are related to each other through the import, use, and refer to relations.

\section{Expert Layer}

Ontologies of the Expert layer are created and maintained by experts of the embedded and security (or any other extra-functional properties) domains. It contains three ontologies. The first two ontologies are obtained from the 


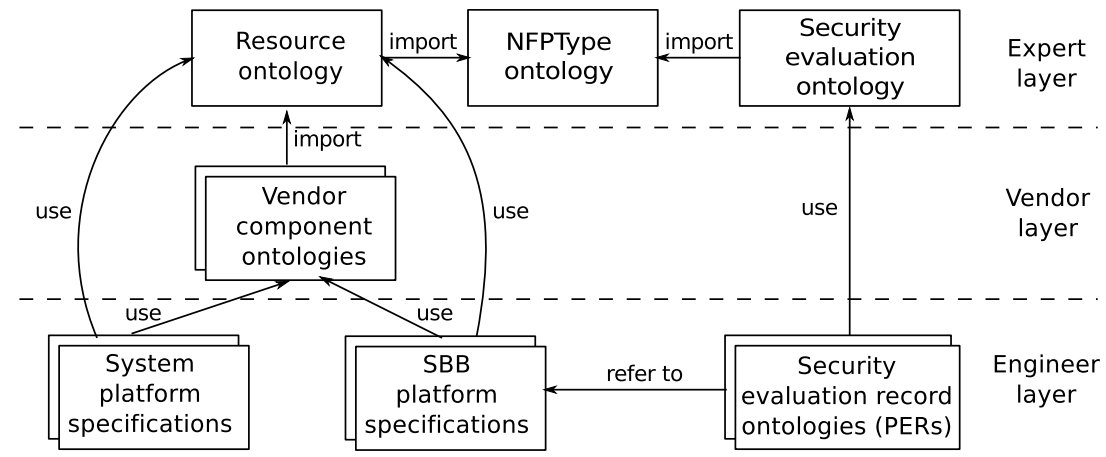

Figure 5.13: Ontologies for compatibility analysis

transformation of MARTE packages dedicated to platform resource descriptions. The third ontology is the refinement of the core evaluation ontology for the security domain as it is already explained in Section 4.1.2.

Techniques to transform UML class models into ontologies are studied and presented by several researchers, e.g. by Hermida et al. [87] and Xu et al. [88]. Similar to their works, we identify the mapping needed to transform MARTE packages. Some basic mapping rules that we apply in our work may be summarised as follows:

- Each MARTE stereotype is represented as an OWL class.

- Each tag is represented as an OWL object or data property, where the domain is the stereotype that owns the tag and the range is the type of the tag. We create an OWL object property if the type of the tag is equal to another stereotype, which can not be replaced with a basic type defined in XSD [89] (e.g. float, integer, or string). Thus, we create an OWL data property if the type of the corresponding tag is a basic XSD type.

- An enumeration is represented as a class with a predefined set of individuals.

- The generalisation relations of the MARTE profile are represented as sub-class relations in the ontology, i.e. the "is-a" relation.

- The composition relations are represented as the part-whole object properties [90], i.e. the "hasPart" relation.

- The named associations are represented as OWL object properties with the corresponding name, e.g. the "hasConnected" relation.

We avoid using the Ontology UML profile [91] that allows designing ontologies as UML models since this requires in-depth understanding of the underlying ontologies from an engineer. Our goal is to exploit advantages 
of ontology technologies (e.g. querying services), but to allow an engineer to operate only with terms of a considered extra-functional domain.

We proceed to explain the remaining two ontologies that build the expert layer, i.e. NFPType ontology and Resource ontology. The third, Security evaluation ontology, was already discussed in Section 4.1.2.

The NFPType ontology of the expert layer contains a set of types and their relations needed to characterise a piece of hardware/software component, e.g. data rate (Mbps, Kbps, etc.) and frequency ( $\mathrm{Hz}, \mathrm{KHz}$, etc.). This ontology is derived from the MeasurementUnits, MARTE_DataTypes, and Basic_NFP_Types sub-packages of the MARTE_Library package (chapter D.2 of the MARTE profile specification), which enable specification of non-functional properties.

Note that types such as NFP_Real and NFP_Integer are not present in our NFPType ontology since they can be sufficiently modelled as the XSD types [89], i.e. xs:float and xs:integer respectively. Figure 5.14(a) depicts an excerpt of the classification from this ontology.

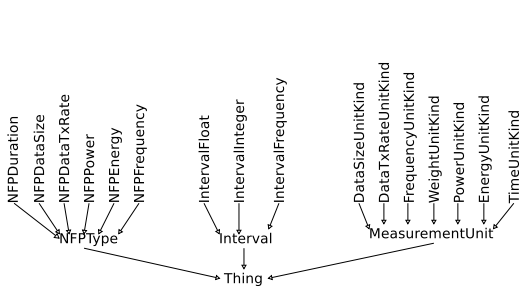

(a) NFPType ontology

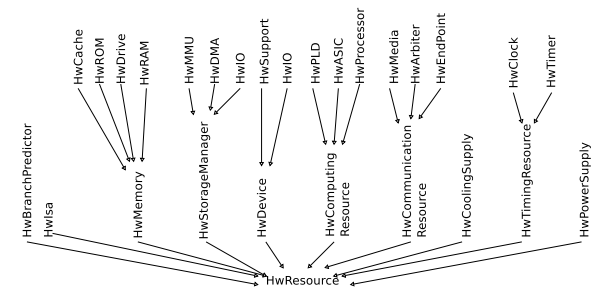

(b) Resource ontology

Figure 5.14: Classification levels for the developed ontologies (excerpts)

The Resource ontology contains those concepts needed to describe platform components and is derived from the MARTE HRM package described in chapter 14.2 of the MARTE profile specification [28]. Some classification levels of this ontology are depicted in Figure 5.14(b).

The core concept of our ontology is the HwResource class that denotes a generic hardware entity. The HRM package differentiates two complementary views onto components, namely logical and physical. This structure is flattened in our ontology since it can be inferred through the ontology view approaches [92].

The logical view (Hw_Logical model) consists of five sub-packages. The core elements of these sub-packages are transformed into sub-classes of the HwResource class. These classes are HwComputingResource, HwIsa, and $\mathrm{HwBranchPredictor}$ from the Hw_Computing sub-package; HwMemory and HwStorageManager from Hw_Storage; HwCommunicationResource from Hw_Communication; HwTimingResource from Hw_Timing; and HwDevice from $\mathrm{Hw} \_$Device. 
The physical view (Hw_Physical model) contains two sub-packages, namely Hw_Power and Hw_Layout. The core concepts of Hw_Power are represented as sub-classes of the HwResource class, i.e. HwPowerSupply and HwCoolingSupply. The Hw_Layout model contains only one element, i.e. HwComponent. This stereotype is used to specify physical characteristics (e.g. weight and area) and environmental conditions (e.g. temperature and humidity) for a platform component. We treat it in a special way in our ontology. Thus, we have encapsulated physical characteristics of a component into the "ComponentCharacteristics" OWL class and related it to the HwResource class with the "hasCharacteristics" object property.

The composition relations defined in HRM are transformed into the "hasPart" object properties [90] (and its inverse property "isPartOf"). The named association "connectTo" is modelled as the "hasConnected" object property (and its inverse property "isConnectedTo"). Both these properties have corresponding sub-properties, e.g. "hasCache" is a sub-property of the "hasPart" property.

\section{Vendor Layer}

The Vendor layer in Figure 5.13 consists of vendor component ontologies, where a vendor is a provider of platform components available for construction of execution platforms for embedded systems (e.g. Texas Instruments [93]). Thus, each ontology encapsulates description of platform components. The resource ontology of the expert layer serves as a description language to describe these components.

In order to provide the description of available components, a vendor needs to perform the following steps. First, a vendor uses the MagicDraw tool [9] to create models of platform components. These models are UML class diagrams annotated with stereotypes from the HRM package. Second, a vendor launches the developed SEED MagicDraw plug-in to transform the created MARTE models of the platform components into an ontology. The user interface of this plug-in is depicted in Figure 5.15(a). We have used the Java OWL API [62] and Acceleo [74] tool to implement this transformation. Figure 5.15(a) depicts an interface of the developed plug-in. A vendor may create a new ontology for described components or add these components into an existing ontology.

Figure 5.4 from Section 5.1.2 depicts a valid model of the OMPA3530 board [81] provided by Texas Instruments.

\section{Engineer Layer}

The bottom layer in Figure 5.13 is the Engineer layer. At this level, system and security engineers use the ontologies created at the higher levels (i.e. the expert and vendor layers) to model the adopted platform for an embedded systems and platforms used for evaluation of concrete SBBs respectively. In 
particular, an engineer uses vendor ontologies when certain vendor components required for the application are known, whereas the resource ontology is suitable if such a component is not known or not present in vendor ontologies. Additionally, security engineers instantiate the security evaluation ontology to capture the results of performance evaluation of a considered SBB. Thus, security engineers create PERs where the concept gemPlatform refers to the $S B B$ platform specification (see Figure 5.13). These PERs are further transformed into corresponding security evaluation record ontologies as explained in Section 4.1.2.

In our smart metering devices case study from Section 2.4, TSMC devices are built on the OMAP3530 board depicted in Figure 5.4. This component will be already described and stored in the vendor ontology. Therefore, an embedded system engineer needs to load the ontology and use this component as a part of the TSMC model. The developed SEED MagicDraw plug-in supports this functionality.

In the measurement transfer scenario of our case study analysed in Section 5.3, the data transmitted between a TSMC and server (i.e. the CollectorToServer asset) must be protected against confidentiality threats as it is indicated by the security property $\mathrm{SP}_{2}$. Consequently, two concrete SBBs that satisfy this security property have been found in the Metering DSSM (see Section 5.4). They are the AES (Advanced Encryption Standard) [94] and DES (Data Encryption Standard) [76] implementations provided by the Texas Instruments [93]. For these implementations, the AES SBB requires the use of the C64x+ processor, while the DES SBB requires the use of the TMS320C6211 chip (see Figure 4.10 in Section 4.1.2).

In the next section, we explain how this architecture of different ontologies enables selection of concrete SBBs (or any RBBs) based on the defined compatibility analysis.

\subsubsection{Model-based Compatibility Analysis}

We differentiate two types of the platform compatibility: logical and environmental. In the following, we explain each of the mentioned types and exemplify some of them using the system and SBB models introduced in the previous sections.

The notion of logical compatibility is based on the pairwise logical compatibility of a SBB and system platform components defined below.

Definition: Two components $A$ and $B$ are logically compatible if one of the following holds: (a) $A$ is identical with $B$; (b) $A$ has $B$ as a part; (c) $A$ is a part of $B$; (d) $A$ can be connected to $B$; (e) $B$ can be connected to $A$; (f) a disjunction of (b)-(e).

We employ the ontology querying services to automate a check of the above definition. In particular, we use the ASK operator of SPARQL [60] that 
returns a boolean value indicating whether a path that matches a query pattern exists. For example, the query for case (b) where the relation "hasPart" is examined has the following form:

PREFIX hrm: [the ontology IRI]

ASK $\{$ ?A hrm:hasPart ?B $\}$

Queries for cases (c) - (e) have a similar structure replacing "hasPart" with the "isPartOf", "hasConnected", and "isConnectedTo" object properties respectively. To support the check of case (f), we use a special construct defined by the SPARQL 1.1 syntax, i.e. the so called path properties [95]. It allows examining a path of an arbitrary length. Hence, the query for case (f) replaces the "hasPart" property with a path expression: (hrm: hasPart | hrm:isPart0f | hrm:connectedTo | hrm:hasConnected)*. In this expression, the symbol "|" denotes the "OR" operator, while the symbol "*" means that any number of occurrences is allowed.

In the query mentioned above, the ?A and ?B symbols denote variables. They are replaced by components of a system platform and components of a SBB platform (annotated with the "gemRequiredComponent" stereotype) respectively. In our case study (see Section 5.5.2), these are OMAP3530 and C64x+ for the AES SBB, and OMAP3530 and TMS320C6211 for the DES SBB. Since TMS320C64x + has a C64x+ processor as its part, the query returns true. In contrast, no path is found between TMS320C6211 and TMS320C64x+ for the DES SBB. Thus, we conclude that the particular implementation of the DES algorithm is not logically compatible with the current design of a system platform that is based on the OMAP3530 board, while AES can be selected as a SBB to provide secure communication for a TSMC device.

The definition for environmental compatibility is built upon the Env_Condition data type from the HRM package (see Figure 14.72 from the MARTE specifications [28]) which defines five types of environmental conditions: temperature, humidity, vibration, shock, and altitude. An environmental condition of each type has a value range. An engineer needs to annotate the components with the "HwComponent" stereotype and define the "r_Conditions" tag to assign environmental conditions to a component. We use the following terms and functions to define environmental compatibility:

- $K$ and $U$ are sets of the environmental condition types and measurement units respectively, where $K=\{$ temperature, humidity, vibration, shock, altitude $\}$ and $U=\left\{{ }^{\circ} C, \%, m / s^{2}, g, m\right\}$.

- A set $E N V \_C O N D$ defined as $I \times U \times K$ that describes each environmental condition as a tuple of a value interval (a set $I$ ), a unit (from the set $U$ ), and a type (from the set $K$ ).

- A function defining environmental conditions of a component, i.e. 
$e n v_{-}$cond $: C O M P \rightarrow 2^{E N V \_C O N D}$, where $C O M P$ is a set of components.

- Projection functions extracting from an environmental condition the corresponding type, i.e. kind: $E N V_{-} C O N D \rightarrow K$, unit, i.e. unit : $E N V \_C O N D \rightarrow U$, and value interval, i.e. range $: E N V \_C O N D \rightarrow I$.

Given these terms and functions we introduce two other functions to define the notion of environmental compatibility.

Definition: Environmental compatibility is a function env_comp : COMP $\times$ $C O M P \rightarrow\{$ true, false $\}$. A component $A$ is environmentally compatible with a component $B$ if $e n v_{-} \operatorname{comp}(A, B)$ is evaluated to true as defined below:

env_comp $(A, B) \triangleq$ true if $\left\{\left\langle\left\langle i_{1}, u, k\right\rangle,\left\langle i_{2}, u, k\right\rangle\right\rangle \mid a \in\right.$ env_cond $(A)$, $b \in \operatorname{env} \_c o n d(B), k \in \operatorname{kind}(a) \cap \operatorname{kind}(b), i_{1}=\operatorname{range}(a), i_{2}=\operatorname{range}(b), i_{1} \cap i_{2} \neq$ $\emptyset\} \neq \emptyset$ or $\neg \exists k: \forall a \in$ env_cond $(A), \forall b \in$ env_cond $(B), k \in \operatorname{kind}(a) \cap \operatorname{kind}(b)$

The intuition is that environmental conditions of a platform component $A$ adopted for an embedded system and a component $B$ required by an SBB are compatible if corresponding interval values of environmental conditions of the same type are overlapping. These components are also compatible if there are no conditions of the same type defined for both components.

In addition, we define another function that specifies the environmental conditions under which a pair of components can not operate (although each could operate individually under respective conditions). We refer to such environmental conditions as environmental constraints. This function is defined as env_constr : $C O M P \times C O M P \rightarrow 2^{E N V_{-} C O N D} \times 2^{E N V_{-} C O N D}$ as follows:

env_constr $(A, B) \triangleq\left\{\left\langle\left\langle i_{1}, u, k\right\rangle,\left\langle i_{2}, u, k\right\rangle\right\rangle \mid a \in\right.$ env_cond $(A), b \in$ env_cond $(B)$, $k \in \operatorname{kind}(a) \cap \operatorname{kind}(b), i_{1}=\operatorname{range}(a) \backslash \operatorname{range}(b), i_{2}=\operatorname{range}(b) \backslash \operatorname{range}(a)$, $u=\operatorname{unit}(a)\}$

The intuition is that while each component might operate in an interval that is a subset of its operational condition, its composition with another component dictates that one component is prohibited from operating in those environment conditions that are not within the range allowed by another component (and vice verse). In other words, environment conditions of one component are constrained because of presence of another component. Therefore, environment constraints for each component are generated. Alternatively, the presence of these constraints for components guides a system engineer to implement a mechanism (e.g. cooling system) that ensures that the corresponding components sustain the environment constraints generated for another component (e.g. to keep within a given temperature range). The SEED MagicDraw plug-in generates these environmental constraints automatically from given environment conditions attached to indi- 
vidual components.

In our case study, the temperature conditions for OMAP3530 and TMS32$0 \mathrm{C} 64 \mathrm{x}+$ (the AES SBB) have the same ranges of $[0 ; 90]^{\circ} \mathrm{C}$. Therefore, the system and SBB are environmentally compatible without any additional constraints.

The above definitions for computing the compatibility relation and their pairwise imposed constraints allow us to reason about environmental conditions of assemblies based on constraints for its constituent components.

Figure 5.15(b) depicts the user interface of the SEED MagicDraw plug-in supporting compatibility analysis. It allows selecting a type of the desired compatibility analysis (logical and environmental) and its settings. The bottom part of this tool shows the results of the analysis, namely whether the system and SBB are compatible according to the selected criteria. Additionally, it shows a generated set of environmental constraints for a environmental compatibility check.

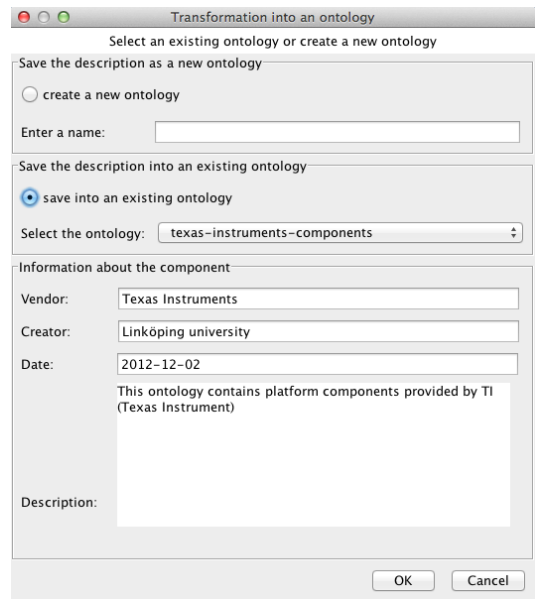

(a) Transformation tool

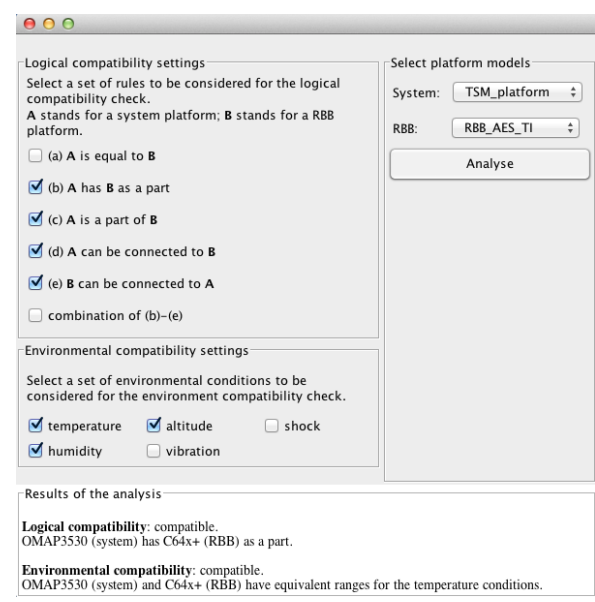

(b) Compatibility analysis tool

Figure 5.15: Model-based compatibility analysis tools (the user interfaces)

\subsubsection{Scalability and Performance}

So far, we have used the case study of smart metering devices to illustrate the compatibility analysis and knowledge management ideas supported by our methods and tool. This section proceeds to show that this approach is scalable to domains with large data sets. We design experiments to estimate the size of resulting vendor ontologies as well as the execution time for the transformation of MARTE models into OWL.

In this study, we focus on microcontrollers (MCUs) provided by some 
of popular vendors (Renesas, Texas Instruments, Fujitsu, Atmel, and Microchip Technology). We estimate the potential complexity of corresponding MARTE models and the size of corresponding OWL ontologies in terms of the number of generated axioms. Three classes of embedded systems and MCUs commonly used for their design [96] are considered: small scale (8-bit MCUs), medium scale (16-bit MCUs), and sophisticated embedded systems (32-bit and ARM-based MCUs). Thereafter, we study how many models are currently available on the market for each vendor (see Table 5.4). The data has been extracted from the official Internet resources of the vendors mentioned above.

Table 5.4: Scalability and performance estimations

\begin{tabular}{l|l|l|l|l}
\hline & & 8-bit & 16-bit & 32-bit \\
\hline 1 & Renesas & 933 & 2290 & 1817 \\
\hline 2 & Texas Instruments & 0 & 406 & 292 \\
\hline 3 & Fujitsu & 103 & 207 & 630 \\
\hline 4 & Microchip Technology & 348 & 334 & 79 \\
\hline 5 & Atmel & 238 & 0 & 179 \\
\hline 6 & Total amount of units & 1622 & 3237 & 2997 \\
\hline 7 & Av. number of axioms per unit & 68 & 105 & 133 \\
\hline 8 & Approx. total number of axioms & 110296 & 339885 & 398601 \\
\hline 9 & Av. transformation time (ms) & 1455.1 & 1627.92 & 2497.92 \\
\hline
\end{tabular}

To estimate the potential number of generated axioms, we select five commonly used MCUs of each class, create their MARTE models, and execute their transformations. This study shows that the simplest 8-bit MCUs creates an average of 68 axioms while the most sophisticated 32-bit MCUs generate 133 axioms (see Table 5.4, row 7). The 8th row shows the approximate number of produced axioms when all models are added into ontologies. Finally, we compare these numbers with scalability studies of the OWL APIs and Jena technologies done by Horridge and Bechhofer [63] and Dibowski and Kabitzsch [92]. In particular, Horridge and Bechhofer [63] show that OWL APIs can easily handle ontologies that contain 1651533 axioms consuming $831 \mathrm{MB}$. As a result, we conclude that the used technologies (OWL APIs and MARTE) allow handling ontologies for a significant number of vendors in a potential real world deployment. This capacity allows loading multiple vendors' ontologies to execute compatibility analysis. Additionally, some techniques for swapping ontologies in memory can be implemented to handle even bigger datasets.

Next, we execute 50 runs of the transformation for the same representatives of each MCU class and measure the execution time for each run (see Table 5.4, row 9). In particular, we measure the execution time of the following operations for each run: transformation of an original UML/MARTE model into the OWL API syntax; generation of axioms executing corresponding OWL APIs; and saving the resulting ontology into an owl-file. The hardware used in this study is a system with $2.8 \mathrm{GHz}$ Intel Core i7 and $8 \mathrm{~GB}$ 
of RAM running Mac OS. In our case, the transformation time does not vary substantially for small and medium MCUs while one second increase is observed for the sophisticated 32-bit MCUs. This increase can be explained by naturally larger, in comparison with 8-bit and 16-bit MCUs, complexity of 32-bit MCUs in both number of elements and their attributes.

\subsection{Extended Form of the Process}

In this chapter, we have presented the process for application of the domainspecific security knowledge that is depicted in Figure 5.1. The process has been intentionally simplified by the author to facilitate explanation of the introduced methods and tools. In this section, we give a broader view to this process.

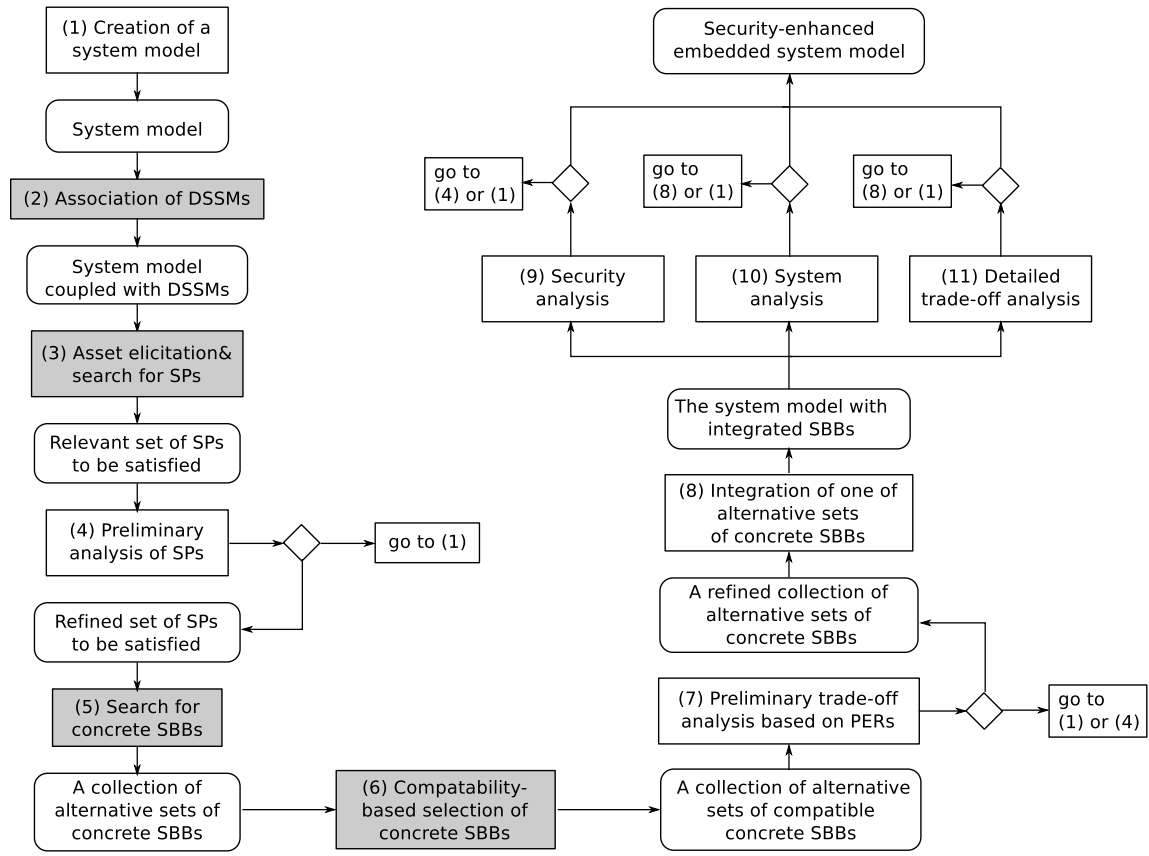

Figure 5.16: Extended form of the proposed process

Figure 5.16 depicts the extended representation of our process. The grey boxes indicate the steps that rest on the SEED methods explained earlier in this chapter. An embedded system engineer starts to create a system model that includes both functional and execution platform models. Thereafter, an engineer proceeds with association of a relevant set of DSSMs (see Section 5.2) followed by application of the asset elicitation technique and search for security properties (SPs) method explained in Section 5.3. When a set of security properties that are relevant for a considered system 
are known, preliminary analysis of the proposed security properties should be conducted as it is shown by the Preliminary analysis of the SPs step. For example, an engineer can go through additional risk analysis to eliminate some assets and associated security properties. Alternatively, satisfiability of the generated set of security properties can be checked. This analysis can result in the necessity to redesign a system as it is indicated by the go to (1) step.

As we have explained in Section 5.4, the Search for concrete SBBs step may lead to proposing a number of alternative ways to secure a system, i.e. a collection of alternative sets of concrete SBBs. The Compatibilitybased selection of concrete $S B B s$ step allows narrowing the initial collection of concrete SBBs sets. Thereafter, the proposed ways (sets of concrete SBBs) to secure a system can be preliminary studied applying some kind of trade-off analyses as indicated by the Preliminary trade-off analysis based on PERs step. Recall that each PER stores information about concrete SBBs performance indices. If this analysis shows that all proposed sets of concrete SBBs can not be sustained by available resources of a system than an engineer can return to step (1), e.g. to redesign a system, or step (4), e.g. to reduce a number of assets.

Thereafter, one selected set of concrete SBBs is integrated into a system design. At this phase, an embedded system engineer can use tools and techniques developed by the research community to conduct a detailed analysis to study consequences of incorporating security functions into a system. The Security analysis step can be used to check whether a set of integrated SBBs provides the required level of security. The System analysis step can assist in verifying if security functions do not violate functional requirements of a system, e.g. all deadlines are met. The Detailed trade-off analysis step allows ensuring that available system resources are enough to execute both system and security functions. Thus, the former step may result in redesigning a system (i.e. go to step (1)) or reconsidering security requirements (i.e. go to step (4)). The latter two tasks may lead to selecting another set of concrete SBBs to be integrated into a system (i.e. go to step (8)) or redesigning a system going to step (1). In the case when all checks outlined above show satisfactory results a system model with integrated set of concrete SBB produce a security-enhanced embedded system model. 


\section{Chapter 6}

\section{Related Work}

In this thesis, we have presented the SEED approach that aims at supporting designing of security-enhanced embedded systems. In the following, we describe some works that are related to our contributions from different perspectives. We discuss those works that target similar objectives or that employ similar methodologies.

\subsection{Composing a System from Reusable Blocks}

The growing complexity of systems determines the need to enable their construction as composition of reusable blocks. This approach brings a range of benefits: it allows reducing the development time and cost, improving the quality of reusable artefact, utilising better expertise of engineers from various domains, to name a few. In our work, we exploit the MBE method SPACE where the notion of a building block is developed. However, there are other widely spread approaches that can potentially support reusability of security solutions across different systems. In this section, we describe two such approaches, namely component-based and aspect-oriented paradigms.

\subsubsection{Component-based Development}

A component is a core concept used in component-based approaches for system development. It is a reusable block that encapsulates some system functions or services. The widely accepted definition of a component is given by Szyperski [97].

"A software component is a unit of composition with contractually specified interfaces and explicit context dependencies only. A software component can be deployed independently and is subject to composition by third parties."

Originally, components are meant to be delivered as binary units deployed and composed at run-time. However, this requirement is often relaxed in case of embedded systems up to the design phase due to an overhead 
created by a component framework [98]. We proceed to describe the basic concepts and terminology used in component-based system development approaches $[99,100]$.

To enable component-based design, each component must adhere a specific component model that defines a set of rules and conventions to describe a component. In particular, a component is defined by its interfaces. Each interface reflects properties of the component that are visible externally (i.e. for other components and a system as a whole). Interfaces can be represented as a set of operations with a list of input and output parameters, i.e. operation-based interfaces. Alternatively, interfaces can be considered as entities that send and receive data, i.e. port-based interfaces. One can distinguish provided (e.g. operations provided for their environment) and required (e.g. operations required from their environment) interfaces. Additionally, the notion of rich interfaces is introduced [99] to refer to interfaces that contain additional information about interfaces, e.g. declaration of their extra-functional properties such as the execution time. Rich interfaces enable certain verifications when composing components.

The process that establishes connection between components (i.e. the composition of their functions) is called component composition, binding [100], or wiring [101]. The result of composition of two or more components is referred to as an assembly. In some component models, the composition of components is supported through connectors that are mediators between components. A component model is supported (at the design- or run-time) by a component framework that is an infrastructure that manages resources for components.

A set of challenges arises when adopting component-based development approaches for embedded systems. For example, the temporal properties of embedded systems require that components provide time characteristics as a part of their interfaces. This is a difficult task if a component is considered to be a software unit since temporal properties depend on the underlying hardware. However, the need to utilise benefits of component frameworks for the world of embedded systems determines the tendency of developing component-based approaches for these domains as well, e.g. AUTOSAR [102] for automotive industry and ROBOCOP [103] for consumer electronics domains. Hošek et al. [104] compare ten other component frameworks focusing on those that provide support for execution. Besides general features related to component models and frameworks (e.g. presence of connectors, type of interfaces) the authors consider a set of requirements that are coming from the domain of embedded systems. They are, for example, support for coupling with hardware or modelling real-time attributes.

Several component frameworks have been enhanced to deal with security aspects. For example, Saadatmand and Leveque [105] develop a methodology to integrate security mechanisms into the ProCom component model. Abdellatif et al. [106] propose a method to enforce an information flow control of the Think component model. 


\subsubsection{Aspect-oriented Development}

Aspect-orientation enforces the separation of concerns principle [107]. This principle promotes identification of different concerns in a system and their separation encapsulating them into reusable artefacts, e.g. modules. These artefacts can be analysed in isolation and applied in several applications. In aspect-oriented modelling (AOM), reusable modules usually realise so called crosscutting concerns. The concern is called crosscutting if a requirement, that it expresses, cuts across a whole system. Thus, reusable modules that implement crosscutting concerns provide such extra-functional properties as security, safety, or other quality of services properties as opposed to traditional functional units of decomposition used in component-based development. The terminology of AOM is not so mature as the terminology of component-based development. However, we have identified some basic concepts used in AOM approaches that deal with the security concern.

A primary system model is a base model of a system (both its functionality and architecture) under development. This primary system model is further extended with a so called aspect that is a reusable implementation of some function that fulfils a crosscutting concern. Each aspect has two forms: a generic aspect is an application independent model of an aspect, and a context-specific aspect is a generic aspect instantiated for a given application. To transform a generic aspect into a context-specific aspect, a set of adaptation rules are applied. Basically, these adaptation rules specify how to map the abstract syntax of a generic aspect into the syntax of a certain application domain. Finally, a context-specific aspect is integrated into a primary system model that results in an integrated system model. The integration is done applying a set of composition rules that can be implemented as an engine that takes primary system model and context-specific aspects as an input and produces an integrated system model. Alternatively, composition rules can be represented as a set of instructions for an engineer that describe steps to be taken for composition. The comprehensive and extensive conceptual reference model of aspect-oriented modelling constituents is presented by Schauerhuber et al. [108]. Below, we overview two AOM approaches that deal with the security and dependability concerns. These approaches employe UML for modelling of a system and aspects.

France et al. [109] represent a generic aspect (referred to as "generalised form of a solution") as a pattern that describes common characteristics of a solution. These patterns are realised as UML model templates. The adaptation is implemented as instantiation of a pattern by binding (i.e. relating) its template parameters to application-specific values. The composition is realised by merging UML models of a primary system model with contextspecific aspects. This merge can be controlled and managed by so called composition directives. For example, composition directives can be used to specify that some elements of a primary system model should be removed, added, or modified in a certain way. They also can predefine the order when several aspects are composed with one primary system model. Thus, vary- 
ing composition directives several (potentially different) integrated system models can be obtained. Further, an integrated system model is analysed to reveal conflicts or undesirable properties, and to investigate whether the used aspect provides the required level of dependability.

Mouheb et al. [110] presents another approach for AOM. A primary system model is a UML model where some elements are annotated with stereotypes and tags that express security requirements, e.g. confidentiality and integrity of data. Additionally, a primary system model is extended with specifications of join points used to support composition of a primary system model and aspects. In particular, join points specify where an aspect should be integrated in a primary system model, e.g. before or after a specific operation. An aspect is a UML class model that is extended with a set of stereotypes from a specific UML profile provided by the authors. For example, one of the stereotypes provided by this profile is a pointcut that is used to specify where in a primary model an aspect should be injected. Similar to join points, a pointcut can specify, for instance, that an aspect should be inserted before or after a UML operation or call. Each aspect can provide several functions that are referred to as advice in the mentioned profile. The adaptation of a generic aspect, i.e. creation of a contextspecific aspect, is done as matching join points from a primary system model and pointcuts from a generic aspect. The composition is defined as actual weaving of aspects into a primary system model.

To sum up, approaches that allow dealing with security in a reusable manner emerge. In these approaches a security measure that enforces security properties is represented in the form of a reusable unit. Each approach has its own syntax and frameworks (i.e. theoretical foundations supported by tools) to integrate a reusable unit (i.e. a component, aspect, or building block) into a system model. In our work, we employ the notion of reusable building blocks used in the MBE method SPACE. Thus, we enjoy a wide range of capabilities provided by this method. They are, for example, a powerful tool-set based on Eclipse Modelling Framework called Arctis [37] and a rich library of already implemented RBBs [38] including the one that model security measures. Moreover, the SPACE RBBs are specified by the modelling language that is based on the well-known syntax of UML activities supported by the formal semantics (Temporal Logic of Actions) [39]. The semantics is suitable for designing of distributed systems built of embedded devices. Moreover, this modelling language allows us to analyse SPACE models to elicit new information required to support integration of a relevant set of security measures.

\subsection{Security-enhanced System Design}

This section summarises our review of methods developed to assist a system engineer in integrating security mechanisms into a system design. We start discussing methods to deal with security aspects that are designed 
for general systems in Section 6.2.1 followed by methods that target embedded systems in Section 6.2.2. Thereafter, Section 6.2 .3 briefly describes approaches related to selection of security measures given a repository of available alternatives. Finally, we briefly discuss some methods to conduct risk analysis in Section 6.2.4 positioning the proposed asset elicitation technique in the context of these methods.

\subsubsection{General Methods to Deal with Security}

The challenge of integrating security mechanisms into various types of systems has been addressed by several approaches. They are, for instance, $\mathrm{MDSE}^{1} / \mathrm{UMLsec} \mathrm{MDS}^{2} / \mathrm{SecureUML}$, security aspects, security patterns, and AVATAR, to name a few. MDSE/UMLsec [111] is one of the first approaches developed for integration of security related information into UML specifications of a system. In particular, UMLsec is a UML profile that is used to incorporate security requirements (such as the fair exchange principle and secure communication links) in the form of corresponding stereotypes (i.e. "fair exchange" and "secure link" respectively) and their tags (e.g. those tags that allow specifying an adversary) into various UML models (e.g. class or activity models). These stereotypes allow a system engineer to specify a proper set of security requirements for a system under development. Thereafter, a system design should be enhanced to meet the augmented security requirements using such techniques as, for example, security patterns [112] or direct refinement of an initial system design. The use of UMLsec allows formally verifying if the resulted system design meets the annotated security requirements. Additionally, there are some works that complement the UMLsec methodology with the security requirements elicitation phase. Thus, Houmb et al. [113] propose a methodology to elicit requirements from stakeholders employing a heuristics-based tool empowered by the common criteria standard [114].

MDS/SecureUML [115] deals with design and verification of role-based access control systems. SecureUML is a security modelling language that should be combined with other languages used for a system design. Thus, it enables a system engineer to model both security and functional aspects of a system simultaneously. The merge of languages is proposed to be implemented through a so called "dialect". A dialect is used to match metamodels and syntax of corresponding functional and security languages. In particular, a dialect shows what elements of a system design language are SecureUML resources and what actions are applied to these resources. Thus, SecureUML can help to express such information as which elements of a system model shall be considered as resources and what actions are permitted under these resources.

The aspect-oriented paradigm presented by France et al. [116] identi-

\footnotetext{
${ }^{1}$ Model-Driven Security Engineering

${ }^{2}$ Model-Driven Security
} 
fies security as a crosscutting concern. To deal with this concern, security functions are encapsulated as aspects that are woven into the initial system model. In this approach, security requirements are elicited by means of composing a system model with a threat model and checking if the attack succeeds. Thereafter, a suitable security aspect is encapsulated into a system design. Aspect-Oriented Risk-Driven Development (AORDD) is an approach developed by Georg et al. [117] that extends the contribution of France et al. [116] to calculate the fitness of different security measures as a trade-off between different criteria, e.g. provided security level, project and deployment effort. The fitness score for a particular security measure is calculated with a specially constructed Bayesian Belief Network. Further, Houmb et al. [118] add to AORDD the performance analysis step employing the PUMA tool.

Security patterns [112] are intended to capture security solutions for common security challenges. In general, a pattern is an extremely broad concept that can be used to encapsulate expert knowledge of any kind (e.g. a reoccurring structure, process, activity, or just some kind of "thing") along all phases of a system development. To be able to cover such diverse information, patterns are defined in a highly generic form. In particular, each pattern describes a solution in a human-readable text (sometimes referred to as patterns's documentation), which is sometimes augmented by UML models to help developers to understand a pattern. Schmidt et al. [119] develop an approach called Security Engineering Process using Patterns (SEPP). In this process, the authors introduce a special type of patterns called Security Problem Frames (SPFs). SPFs consider generic security requirements omitting possible means to satisfy these requirements. The SPF description contains the following fields: name, intent, a frame diagram, informal description, a security template, and an effect. Each field is defined in a textual form, as a UML model, or in some other formal notation. Thereafter, the authors introduce the notion of a Concretised Security Problem Frame (CSPF) that describes generic security measures linked to related SPFs. It is the responsibility of a system engineer to select and instantiate both SPFs and CSPFs to proceed with the SEPP method.

Other approaches that are designed to assist a system engineer to secure a system can also be mentioned. For example, Hamid et al. [120] enforce the notion of security (and dependability) patterns supported by formal validations. Pedroza et al. [121] propose a SysML-based environment called AVATAR to model and verify safety, authenticity, and confidentiality properties. The recent work of Uzunov et al. [122] provides a comprehensive survey, comparison, and classification of many other methodologies to assist in designing security-enhanced systems.

Compared with the SEED approach proposed in our work where the security-related knowledge is captured by DSSMs, the approaches mentioned above still require in-depth security knowledge from a system engineer or presence of a security expert. For example, it is not clear how a system engi- 
neer should select a suitable security aspect in the AORDD method. It is assumed as the given knowledge since the authors know a priori how to model relevant attacks and how to construct and weave a security aspect. A security pattern is very powerful concept to assist in analysing and developing secure software in a systematic manner exploiting the "body of accumulated knowledge" represented as a pattern. However, due to its unfixed (though flexible) syntax, their application is thought to be manual. This statement is supported by the fact that security patterns, in general, do not make any assumption on the used design language for a system implementation. Thus, application of current security patterns can be hardly automated. In the SEPP method, selection and instantiation of both SFPs and CSPFs are supposed to be done by a system engineer that can be cumbersome due to their complicated structure. In contrast, we provide a bridge between security domain experts and embedded system engineers. The SEED approach defines a structured methodology (i.e. concepts, methods, processes, and tools) to create and select a suitable set of security measures represented in the form of reusable building blocks that can be integrated into a system under development. Thus, our approach produces a guideline on where and why a specific security measure should be applied. Moreover, SEED allows separating the task of designing a security mechanism from a system design.

\subsubsection{Methods for Embedded Systems}

A number of model-based approaches to enforce security within embedded systems are proposed. The Ruiz et al. [123] approach requires that a system engineer defines a set of security properties for a scenario. This assumption already requires that an embedded system engineer has a good expertise in security. According to Ruiz et al., each security property is further linked to attacks and threats. Attacks and threats are modelled in order to describe the capabilities of intruders to cause harm in a system. Finally, these threat models are associated with a set of static and dynamic tests to enable the later testing of a system integrated with security mechanisms.

In our approach, we propose a method to systematically elicit required security properties consulting the security knowledge captured in DSSMs. Therefore, our approach addresses the situation when an embedded system engineer has just limited expertise in security. The knowledge about threats is indeed exploited in our approach to refine a set of initially elicited security properties. We believe that our approach can be complemented by the Ruiz et al. [123] idea to link threats with a corresponding set of tests. However, we envisage that there is also a need to extend this idea linking security properties and threats to verification facilities (besides tests). This enhancement will provide required assurance already at the design phase as opposed to postponing all checks until the first prototype of a system is available when testing can be performed [124].

Hamid et al. [125] attempt to model trust properties as reusable patterns 
for specific domains. While that work shares our aspirations for reusability, we consider security concerns rather than trust relations. Similar to our work, Eby et al. [126] adopt principles of domain-specific modelling. They propose to integrate a Security Analysis Language (SAL) into a DSML for the embedded systems domain (that reminds us the SecureUML approach). However, they focus on security of information flows.

Saadatmand and Leveque [105] develop a method for incorporating security aspects into the ProCom component model. The authors consider two security goals, namely confidentiality and authentication. This approach proposes to use (manual) annotations to identify those parts of a system model where integration of security aspects is needed. In contrast to this work, we have developed the asset elicitation technique to identify vulnerable parts of a system avoiding manual tagging of a system model.

\subsubsection{Selection of Security Measures}

An important step that precedes actual integration of security building blocks is the selection of such. We have observed that the model-based and model-driven methods mentioned above do not address this task in detail. However, approaches for selection of security measures are under development in the context of security patterns [112].

Selection of security patterns is based on their classification. Such classification can be built of just three classes (Fernandez et al. [127]) or be represented as a multi-dimensional matrix of classes (VanHilst et al. [128]) including such categories as an architectural layer (e.g. network), a lifecycle stage (e.g. design), and a domain (e.g. enterprise systems). In our work, the basics for selection of concrete SBBs are domains, security goals, defence strategies, and assets. Thus, there are some overlapping concepts in our work compared to the dimensions elaborated for security patterns. However, SEED proposes that only the domain dimension is selected by an embedded system engineer following the guideline. Selection of other categories is done by applying the elicitation technique and querying the core security ontology.

Hafiz et al. [129] organise security patterns according to the CIA ${ }^{3}$ goal model, a pattern's application context (i.e. core security, perimeter security, and exterior security), a problem domain, and their classification based on the STRIDE $^{4}$ model. All these classifications are formalised in tabular or tree forms. In addition to this method, Washizaki et al. [130] develop the Dimensional Graph (DG) concept to formalise the multi-dimension classification of security patterns. Each DG uses a UML object diagram to show relations of a pattern to a set of dimensions of interest. We formalise our categories as the core security ontology, which allows utilising advantages of

\footnotetext{
${ }^{3}$ Confidentiality, Integrity, and Availability

${ }^{4}$ Spoofing, Tampering, Repudiation, Information disclosure, Denial of service, and Elevation of privilege
} 
the ontology technology querying services for the selection of concrete SBBs.

\subsubsection{Risk Analysis}

Asset identification is an essential stage of risk analysis methods that precedes selection of security mechanisms, e.g. as in the CORAS model-based method [84] or such general frameworks as OCTAVE (Operationally Critical Threat, Asset, and Vulnerability Evaluation) [131] and CRAMM (CCTA Risk Analysis and Management Method) [132]. Identification of assets is normally done through some informal analysis (implemented as brainstorming sessions or interviews) of a system model or other (often informal) representations. We have proposed to automate this task in the presence of a formal system model composed of functional and execution platform views.

We have defined the rules for traversing a system model expressed as SPACE collaborative activities to identify assets and classify them in accordance with the knowledge captured within the security ontology. Thereafter, the knowledge about vulnerabilities and threats associated with elements of MARTE platform models is applied to refine the initial set of assets. Note that our methodology does not deny the best of the practice methods existing today. Thus, for example, threat diagrams of the CORAS method can be used to identify those threats that expose components of an execution platform adopted for a system under development. Alternatively, threat and vulnerability security assessment patterns [112] can be employed for this purpose.

Moreover, the asset identification phase of risk analysis methods and frameworks $[84,131,132]$ usually involves both security experts and stakeholders that are embedded system engineers in our case. In our work, we undertake one step forward and emphasise the need to make results of such analysis once conducted by security experts reusable for embedded system engineers by means of storing its outcomes in a suitable form, i.e. as ontologies, and providing methods for embedded system engineers to apply this knowledge when designing a system.

\subsection{Marrying Ontologies and Models}

The use of ontologies to support tasks of model-driven engineering is an interesting research topic [133]. The potential of ontology technologies applied to the system and software engineering to formalise general modelling is outlined by Tetlow et al. [134]. Recall that ontologies are used to represent knowledge as a set of domain concepts and their relations. Similarly, the conceptual description of a domain through meta-modelling is performed while creation of a DMSL [25]. Both technologies suggest a range of benefits. For example, one of the main benefits of the ontology technology is its automated querying services while DSMLs enjoy wider adoption in development environments and tools. Therefore, it is not a surprise that researchers 
try to find a logical synergy to exploit advantages of both of these technologies [133]. For example, Walter et al. [135] in their recent work employ ontologies to improve the practice of domain-specific modelling (DSM). The authors have developed a framework for DSMLs that relies on the ontology reasoning services (e.g. the inconsistency checker) to guide a designer and to validate incomplete structural domain models. In our work, we combine the ontology and DSM technologies to assist the development of securityenhanced system models from different application domains. We employ DSM to constantly populate the used ontology with the domain-specific security knowledge. Additionally, we provide ontological support for the standard MARTE profile.

Another interesting and active topic is the use of ontologies to facilitate designing of complex systems. Dibowski et al. [92] present the ontological framework to describe devices for the building automation domain. Jianjun et al. [136] use ontology to configure embedded control systems based on a functional model of a system that is intended to express the user demand. Wagelaar [137] combines the ontology technology with the model-driven architecture principles to enable reuse of platform-independent to platformspecific models (PSMs) transformations. Tekinerdoğan et al. [138] employ ontologies to support selection of PSMs, where a system platform is described as a set of high level properties. We use ontologies to support a composition of embedded systems from RBBs that provide extra-functional properties. Furthermore, we use MARTE models and extend them with additional concepts to formulate platform-specific constraints as a basics for such composition. Specifically, we elaborate on the notion of model-based compatibility as one of possible criteria for selection of a set of concrete SBBs where security is an example of an extra-functional domain.

\subsection{Performance Analysis at the Design Phase}

There is a range of tools that enable performance analysis when only a design of a system is available. Robert and Perrier [139] and Piel et al. [140] present CoFluent and Gaspard2 methodologies and tools to execute performance analysis at the design phase. These techniques use UML/MARTE models to describe architecture and application designs. In addition to the MARTE profile, CoFluent employs the SysML [141] modelling language. Both CoFluent and Gaspard2 introduce some constraints on the original semantics and on usage conventions of MARTE models to enable their further analysis. Similarly, we make some assumptions on MARTE models when designing the support (methods and tools) for model-based compatibility analysis. Thereafter, both CoFluent and Gaspard2 methodologies use a different chain of transformations to generate SystemC TLM ${ }^{5}$ code for its further simulation in suitable tools.

\footnotetext{
${ }^{5}$ Transaction Level Model
} 
The use of performance analysis while composing a system with RBBs at the early design phases is a subject of active research. Woodside et al. [142, 143] develop and apply the PUMA ${ }^{6}$ approach for performance analysis of RBBs represented as security aspects. Woodside et al. start exploiting the UML SPT ${ }^{7}$ profile, but their further works adapt this methodology for the MARTE profile. In this work, the authors generate LQN ${ }^{8}$ models that are analysed by a solver and simulator. Similarly, Wehrmeister et al. [144] presents the AMoERT ${ }^{9}$ methodology when the aspect-oriented paradigm is used. In this work, the authors propose the GenErTiCa tool to generate Java code for a specific predefined (though selected by an engineer) target platform. Bondarev et al. [145] present the CARAT ${ }^{10}$ toolkit for performance evaluation where RBBs conform to a specific component model. The authors use their own modelling language to describe the application logic and architecture that are synthesised into an executable system model used for the task scheduling.

We can distinguish two categories of methods that allow obtaining performance evaluation results when only a design model is available. The methods of the first group (CoFluent and AMoERT) take a set of application and platform models as an input and generate code, e.g. C and SystemC. Afterwards, the generated code is executed in a simulation tool. The methods of the second group (PUMA, CARAT, and Gaspard2) use models as a means to input required data into analytical performance analysis tools.

The SPACE method employed in our work enables code generation for functional models (i.e. UML activities) where Java code is produced from state machines for a certain predefined execution platform (e.g. ServiceFrame [39]). Thus, one can relate the SPACE methodology to the first category of the classification outlined above excepting the fact that transformations are bound to a specific predefined execution platform. In our work, we complement SPACE models with an execution platform described as MARTE models. These MARTE models can be converted into the corresponding simulation code using methods developed in the approaches mentioned above. Therefore, there is a potential to simulate SPACE models on a desired execution platform (that is modelled in MARTE), once a formalised and tooled allocation of SPACE models onto MARTE models is defined. Moreover, our work complements the approaches described above since it enables reuse of outcomes of SBBs performance evaluation conducted by corresponding domain experts.

Ciccozzi et al. [146] propose a meta-model used to propagate results of monitoring extra-functional properties at the code level back to a system model in order to improve it. This approach is developed for the CHESS modelling language [147]. In our work, we exploit MARTE models to cap-

\footnotetext{
${ }^{6}$ Performance by Unified Model Analysis

${ }^{7}$ Schedulability, Performance and Time

${ }^{8}$ Layered Queueing Networks

${ }^{9}$ Aspect-oriented Model-Driven Engineering for Real-Time systems

${ }^{10}$ Component Architectures Analysis Tool
} 
ture platform-specific constraints of embedded systems and SBBs. The proposed MARTE compatible profile allows capturing more information about outcomes of SBBs performance analysis, e.g. the used workload and the observed resource footprint. This information (structured, captured, and searchable) aids an embedded system engineer to select an appropriate SBB to satisfy required security (or other extra-functional) properties. 


\section{Chapter 7}

\section{Conclusions and Future Work}

This thesis has presented the SEED approach that supports embedded system engineers and security experts in designing security-enhanced systems. This chapter summarises this work and discusses some interesting directions for the future work.

\subsection{Conclusions}

The increased use of embedded systems in countless applications has led to a boost in their complexity and a need for constant connectivity to open networks. As a result, these systems operate with different categories of data which often include sensitive information. In realities, the task of assuring security properties of embedded systems becomes more challenging, which immediately leads to an emergent need in specific methods and tools to support the development of embedded systems.

In this work, we have focused on some aspects of the aforementioned challenge and identified the basic principles currently developed by the research community to address these aspects. These aspects and principles are outlined below. First, the complexity and resource scarcity of embedded systems can no longer sustain the practice of adding security measures at the late development phases. To overcome this issue, the focus on security should be shifted to the design phase following the principles of modelbased engineering. Second, although security attracts more attention, there are still a fewer security experts than embedded system engineers. The need to share the knowledge of the security experts and apply it in multiple domains in an effective way emerges. We tackle this issue by implementing the principle of separation of concerns. Finally, complexity of embedded systems and modern security solutions encourages that security solutions 
are adjusted to a certain application domain, so that their integration into an embedded system design is less of a burden. We exploit the principle of domain specialisation to address this issue.

To address the named challenge, we have developed an approach called SEED that is built around the basic principles mentioned above. With the help of SEED, security experts gain an opportunity to describe developed security solutions in a reusable manner and embedded system engineers can select a suitable set of security solutions based on an analysis of both system's security needs and its resource constraints.

SEED rests on two concepts introduced in this thesis, namely DomainSpecific Security Model (DSSM) and Performance Evaluation Record (PER). The DSSM and PER concepts described in Chapter 4 are relatively easy to use since they are UML models that are formalised as ontologies: the core security ontology and the core evaluation ontology respectively. These concepts serve as tools for security experts to capture their knowledge about existing security solutions. Each DSSM enables characterising common security issues of a specific application domain in a form of security properties. Thereafter, security properties are linked to available solutions that can be used for their enforcement. Each PER is used to characterise the resource overhead created by a security measure, quality of provided extra-functional properties, and an evaluation technique applied. The underlying ontologies allow storing the knowledge provided by experts and inspecting this knowledge when it is needed for embedded system engineers.

Additionally, the SEED approach is combined with a set of methods and tools that support an embedded system engineer in selecting a suitable set of security measures to be integrated into a system design. These methods, described in Chapter 5, assist an engineer to consistently use the knowledge provided by security experts. The first method, called asset elicitation technique, allows analysing a system design and identifying its security needs by consulting DSSMs. The method conducts inspection of functional and execution platform models, represented as SPACE and MARTE models respectively, and consult DSSMs to obtain a set of security properties that are recommended for implementation. A set of security solutions that satisfy security properties are also retrieved from DSSMs. The second method developed in this thesis examines resource constraints of security solutions stored in PERs. This technique, called model-based compatibility analysis, matches platform-related constraints of a system under development and those required by security solutions.

The SEED approach is supported by a set of tools that have been integrated into the MagicDraw environment as a plug-in. These tools use technologies provided by model-driven engineering, e.g. modelling and transformation facilities.

Using the metering devices domain as an example, we have shown that the introduced concepts (DSSM and PER) can be employed by a security expert to describe the security knowledge. Consequently, we have demon- 
strated that it can be used to support an embedded system engineer to integrate a suitable set of security solutions exploring this security knowledge. This case study that is currently under industrial development has been provided as a use case within the EU FP7 SecFutur project.

The SEED approach developed in this thesis has the potential to contribute to the emerging practice of systematic treatment of security aspects in embedded systems. It is one more step towards providing support to embedded system engineers when designing security-enhanced systems. We conclude with a few reflections about the problem and solution spaces investigated during this work.

Our work rests on the premise that security functions encapsulated into reusable SBBs are basic elements used to support designing of securityenhanced embedded systems. However, it is possible to envisage that enforcement of security properties in an embedded system is not limited to the task of incorporating SBBs. In particular, we find that realisation (at the design phase) of the system functionality itself and a choice of the hardware/software components for an execution platform have also a considerable impact on security properties. For example, if several alternative ways to allocate security assets on available components exist, the selected allocation may affect security of a system when components differ in terms of their tamper resistance. This, in turn, affects a range of SBBs selected for their integration into an embedded system. While these aspects might be well understood by security experts, there is a shortage of support for embedded system engineers to adopt them when designing a system. We believe that this knowledge possessed by security experts made available for reuse will empower system engineering teams to be more security conscious.

In Chapter 5 we conclude that the use of DSMLs can improve the asset elicitation technique thanks to richer semantics tailored to an application domain. On the other hand, the use of a specific DSML will limit applicability of SEED (or any other approach) confining it to a certain domain. To increase applicability of DSMLs for general approaches is an important challenge. We believe that one way to address it is to elaborate suitable abstraction layers that will allow adapting an approach for a range of DSMLs. To achieve this flexibility we have structured SEED in two levels of abstraction, namely foundation and realisation. The foundation level is the technology-ignorant level that defines basic principles. The realisation level refines the formulated principles according to a selected DSML.

In conclusion, we must mention that there are still other processes where an embedded system is not composed from separate functional and nonfunctional elements, e.g. building blocks. These processes may have many other emerging challenges when dealing with a task of supporting engineers in developing security-enhanced embedded systems. This is also an interesting direction of work, but it is outside of the scope of this thesis. 


\subsection{Future Work}

Since the scope of our work is fairly broad that opens many aspects with respect to designing a security-enhanced embedded system, many diverse directions for future work can be identified. In the following, we outline some of them.

The current version of the core security ontology refines the asset concept by two sub-classes, namely data in transit and data stationary. One path for future work is to study what are other important assets to be included into the ontology. However, once new assets are introduced, they should be traceable into a system model to automate their elicitation. The currently employed SPACE modelling language is a general language for modelling of distributed applications. Its semantics does not allow identifying other classes of assets within functional models specified with this language. Therefore, a more expressive, i.e. more domain-specific, language should be employed for the SEED realisation. This language should allow identifying newly introduced assets automatically from functional and platform models of a system. Thus, this direction will require studying both a range of possible assets and existing DSMLs analysing their suitability for an extension of the asset elicitation technique.

The representation of security properties is an active and interesting topic. In our work, a tuple consisting of the protected asset, provided security goal, and used defence strategy represents a security property. This convention limits the range of considered security properties to those that can be expressed by such a tuple. However, some security properties can be only expressed, for example, with respect to an operation and an involved actor. Therefore, we think that employment of a more powerful language to express security properties will increase the applicability of the SEED approach. Thus, we propose this topic as another potential direction for future work.

In SEED, we propose to constantly update the enriched security ontology when capturing the domain-specific security knowledge. Therefore, an important question of maintaining consistency of the enriched ontology arises. In particular, an obvious problem with such an update is pollution of the ontology with concrete SBBs that have different names but refer to the same implementation. Some issues can be resolved by built-in ontology services together with such constructs as owl:sameAs or owl:differentFrom. However, additional support is needed to ensure that two (or more) concrete SBBs under different names are equal implementations as an example. We envisage that techniques from the area of model comparison or models diff (applied to functional and platform models of concrete SBBs) can be employed to address the mentioned issue.

The starting point of SEED is when an embedded system engineer has an initial version of a functional model of a system and some decisions about a used platform are taken. However, for security-critical systems, e.g. defence 
applications, the security requirements drive and affect the functionality of a system. Therefore, identifying alternative model-based approaches that start directly from security assets and their security goals can be another subject to study.

The SEED approach provides an infrastructure for capturing diverse performance evaluation results where both resource footprint indices and quality of service characteristics are stored. The natural development of this direction is the use of this data for different kinds of trade-off analyses. Thus, a path for future work will be the exploration of other criteria and strategies to reuse the performance analysis results stored within PERs.

Finally, availability of advanced and engineer-friendly tools is always a question when it comes to engineering processes. In our work, we use MagicDraw as a platform for our supporting tools since it is selected as an integrating environment within the SecFutur project. However, modeldriven engineering increasingly prioritises open standards. Therefore, the migration to such environments as EMF can be a potential thread for future work. Additionally, SEED currently relies on UML object models as a concrete syntax for DSSMs. Development of a text-based syntax can further improve the provided tool support. 



\section{Bibliography}

[1] Peter Marwedel. Embedded System Design: Embedded Systems Foundations of Cyber-Physical Systems. Springer, 2011.

[2] Markus Voelter, Sebastian Benz, Christian Dietrich, Birgit Engelmann, Mats Helander, Lennart C. L. Kats, Eelco Visser, and Guido Wachsmuth. DSL Engineering - Designing, Implementing and Using Domain-Specific Languages. dslbook.org, 2013.

[3] Frank Alexander Kraemer. Engineering Reactive Systems: A Compositional and Model-Driven Method Based on Collaborative Building Blocks. PhD thesis, Norwegian University of Science and Technology, August 2008.

[4] Charles Q. Choi. Cisco IP Phones Vulnerable. http://spectrum.ieee.org, 2013.

[5] Peter Clarke. Embedded Systems Next for Hack Attacks. http://eetimes.com, 2013.

[6] Srivaths Ravi, Anand Raghunathan, Paul Kocher, and Sunil Hattangady. Security in Embedded Systems: Design Challenges. ACM Transactions on Embedded Computing Systems, 3(3):461-491, August 2004.

[7] Marco Brambilla, Jordi Cabot, and Manuel Wimmer. Model-Driven Software Engineering in Practice. Morgan \& Claypool Publishers, 2012.

[8] Paul Kocher, Ruby Lee, Gary McGraw, and Anand Raghunathan. Security As a New Dimension in Embedded System Design. In Annual Design Automation Conference (DAC), pages 753-760. ACM, 2004.

[9] MagicDraw. www.magicdraw. com, last visited May 2013.

[10] The SecFutur project: Design of Secure and Energy-efficient Embedded Systems for Future Internet Application. http://www . secfutur . eu. 
[11] James K. Peckol. Embedded Systems: A Contemporary Design Tool. John Wiley \& Sons, 2007.

[12] Alan M. Davis. Software Requirements: Analysis and Specification. Prentice Hall Press, 1990.

[13] German Ministry of Defense. V Model: Software Lifecyce Process Model, Geenral Reprint No 250., 1992.

[14] Barry W. Boehm. A Spiral Model of Software Development and Enhancement. IEEE Computer Society Press, 1988.

[15] Bruce Powel Douglass. Real Time UML Workshop for Embedded Systems. Elsevier, 2007.

[16] Bran Selic. The Pragmatics of Model-driven Development. IEEE Computer Society Press, 20:19-25, 2003.

[17] Object Management Group. Unified Modeling Language: Superstructure, version 2.4.1, August 2011. Document number: formal/2011-0806.

[18] Jean Bézivin. On the Unification Power of Models. Software and System Modeling, 4:171-188, 2005.

[19] Object Management Group. http://www.omg .org, visited July 2013.

[20] Object Management Group. Meta-Object Facilities: Core Specification, version 2.0, 2001.

[21] Dragan Gasevic, Dragan Djuric, and Vladan Devedzic. Model Driven Engineering and Ontology Development. Springer, 2009.

[22] Krzysztof Czarnecki and Simon Helsen. Feature-based Survey of Model Transformation Approaches. IBM Syst. J., 45(3):621-645, 2006.

[23] Hans Vangheluwe. Invited talk: Promises and challenges of modeldriven engineering. In European Conference on Software Maintenance and Reengineering (CSMR), pages 3-4, 2011.

[24] Holger Giese, Tihamér Levendovszky, and Hans Vangheluwe. Summary of the Workshop on Multi-Paradigm Modeling: Concepts and Tool. In Models in Software Engineering, pages 252-262, 2007.

[25] Steven Kelly and Juha-Pekka Tolvannen. Domain-Specific Modeling: Enabling Full Code Generation. John Wiley \& Sons, 2008.

[26] François Lagarde, Huáscar Espinoza, François Terrier, and Sébastien Gérard. Improving UML Profile Design Practices by Leveraging Conceptual Domain Models. In IEEE/ACM International Conference on Automated Software Engineering (ASE), pages 445-448, 2007. 
[27] Bran Selic. A Systematic Approach to Domain-specific Language Design Ising UML. In Object and Component-Oriented Real-Time Distributed Computing (ISORC), pages 2-9, 2007.

[28] Object Management Group. UML Profile for MARTE: Modeling and Analysis of Real-Time Embedded Systems, version 1.1, June 2011. Document number: formal/2011-06-02.

[29] Charles Andréand Frédéric Mallet, Aamir Mehmood Khan, and Robert de Simone. Modeling SPIRIT IP-XACT in UML MARTE. In Design, Automation 6 Test in Europe (DATE), 2008.

[30] Jochen Zimmermann, Oliver Bringmann, Joachim Gerlach, Florian Schaefer, and Ulrich Nageldinger. Holistic System Modeling and Refinement of Interconnected Microelectronic Systems. In Design, Automation $\&$ Test in Europe (DATE), 2008.

[31] Muhammad Zohaib Iqbal, Shaukat Ali, Tao Yue, and Lionel Briand. Experiences of Applying UML/MARTE on Three Industrial Projects. In Springer, editor, Model Driven Engineering Languages and Systems (MODELS), pages 642-658, 2012.

[32] Simona Bernardi, José Merseguer, and Dorina C Petriu. Adding Dependability Analysis Capabilities to the MARTE Profile. In Model Driven Engineering Languages and Systems (MODELS), pages 736$750,2008$.

[33] Daniela Cancila, Francois Terrier, Fabien Belmonte, Hubert Dubois, Huáscar Espinoza, Sébastien Gérard, and Arnaud Cuccuru. SOPHIA: a Modeling Language for Model-Based Safety Engineering. In Architecting and Construction of Embedded Systems Workshop (ACESMB), 2009.

[34] Huáscar Espinoza, Sébastien Gérard, Henrik Lönn, and Ramin Tavakoli Kolagari. Harmonizing MARTE, EAST-ADL2, and AUTOSAR to Improve the Modelling of Automotive Systems. In ADAMS Workshop hosted by ECRTS, 2009.

[35] ADAMS project: Action for the Dissemination and Adoption of the MARTE and Related Standards for Component-based Middleware. http://www . adams-project.org, last visited July 2013.

[36] Sébastien Demathieu, Frédéric Thomas, Charles André, Sébastien Gérard, and François Terrier. First Experiments Using the UML Profile for MARTE. In International Symposium on Object Oriented RealTime Distributed Computing (ISORC), pages 50-57, 2008.

[37] Frank Alexander Kraemer, Vidar Slåtten, and Peter Herrmann. Tool Support for the Rapid Composition, Analysis and Implementation of 
Reactive Services. Journal of Systems and Software, 82(12):2068-2080, December 2009.

[38] Frank Alexander Kraemer and Peter Herrmann. Automated Encapsulation of UML Activities for Incremental Development and Verification. In International Conference on Model Driven Engineering, Languages and Systems (MoDELS), volume 5795 of LNCS, pages 571585. Springer, 2009.

[39] Frank Alexander Kraemer and Peter Herrmann. Reactive Semantics for Distributed UML Activities. In Formal Techniques for Distributed Systems (FMOODS/FORTE), LNCS, pages 17-31. Springer, 2010.

[40] Frank Alexander Kraemer and Peter Herrmann. Formalising Collaboration-oriented Service Specifications Using Temporal Logic. In Networking and Electronic Commerce Research Conference, 2007.

[41] Linda Ariani Gunawan, Peter Herrmann, and Frank Alexander Kraemer. Towards the Integration of Security Aspects into System Development using Collaboration-Oriented Models. In Dominic Ślȩzak, Tai hoon Kim, Wai-Chi Fang, and Kirk P. Arnett, editors, International Conference on Security Technology (SecTech), volume 58 of Communications in Computer and Information Science, pages 72-85. Springer Berlin Heidelberg, 2009.

[42] Linda Ariani Gunawan, Frank Alexander Kraemer, and Peter Herrmann. A Tool-Supported Method for the Design and Implementation of Secure Distributed Applications. In Engineering Secure Software and Systems (ESSoS), LNCS, pages 142-155. Springer-Verlag, 2011.

[43] Linda Ariani Gunawan and Peter Herrmann. Compositional Verification of Application-Level Security Properties. In Engineering Secure Software and Systems (ESSoS), LNCS, pages 75-90. Springer-Verlag Berlin Heidelberg, 2013.

[44] Eclipse Modeling Framework Project (EMF). http://www.eclipse. org/modeling/emf/, last visited July 2013.

[45] Kermeta. http://www. kermeta.org, last visited July 2013.

[46] Frédéric Jouault and Jean Bézivin. KM3: a DSL for Metamodel Specification. In IFIP WG 6.1 international conference on Formal Methods for Open Object-Based Distributed Systems, pages 171-185, 2006.

[47] Graphiti - a Graphical Tooling Infrastructure. http://www.eclipse. org/graphiti/, last visited July 2013.

[48] Graphical Modeling Project (GMP). http://www.eclipse.org/ modeling/gmp/, last visited July 2013. 
[49] Xtext. http://www.eclipse.org/Xtext/, last visited July 2013.

[50] Enterprise Architecture. http://www.sparxsystems.com/products/ ea/index.html, last visited July 2013.

[51] Rhapsody. http://www-03.ibm.com/software/products/us/en/ ratirhapfami/, last visited July 2013.

[52] MDT-UML2Tools. http://wiki.eclipse.org/MDT-UML2Tools, last visited July 2013.

[53] Papyrus project. http: //www . papyrusuml.org, last visited July 2013.

[54] Meta Object Facility (MOF) 2.0 Query/View/Transformation (QVT). http://www .omg. org/spec/QVT/, last visited July 2013.

[55] ATL. http://www.eclipse.org/atl/, last visited July 2013.

[56] Henshin. http://www .eclipse.org/henshin/, last visited July 2013.

[57] EMorF. http://www.emorf.org, last visited July 2013.

[58] B. Chandrasekaran, John R. Josephson, and V. Richard Benjamins. What are Ontologies and Why Do We Need Them? In Intelligent Systems, volume 14, pages 20-26. IEEE, 1999.

[59] Boris Motik, Peter F. Patel-Schneider, and Bijan Parsia. OWL 2 Web Ontology Language Structural Specification and Functional-Style Syntax, http://www.w3.org/TR/ow12-syntax/, last visited January 2013.

[60] Eric Prud'hommeaux and Andy Seaborne. SPARQL Query Language for RDF. http://www.w3.org/TR/rdf-sparql-query/, last visited January 2013.

[61] Protégé Editor. http://protege.stanford.edu, last visited March 2013.

[62] The OWL API. http: //owlapi.sourceforge.net, last visited March 2013.

[63] Matthew Horridge and Sean Bechhofer. The OWL API: A Java API for OWL ontologies. Semant. web, IOS Press, 2011.

[64] SPARQL in Protégé-OWL. http://protege.stanford.edu/doc/ sparql/, last visited March 2013.

[65] Apache Jena project. http://jena.apache.org/, last visited February 2013.

[66] OWL Web Ontology Language. http://www.w3.org/TR/owl-ref/, last visited July 2013. 
[67] Almut Herzog, Nahid Shahmehri, and Claudiu Duma. An Ontology of Information Security. Journal of Techniques and Applications for Advanced Information Privacy and Security, IGI Global, pages 278301, 2007.

[68] Stefan Fenz and Andreas Ekelhart. Formalizing Information Security Knowledge. In ACM Symposium on Information, Computer and Communications Security (ASIACCS). ACM, 2009.

[69] Anya Kim, Jim Luo, and Myong Kang. Security Ontology for Annotating Resources. In International conference on On the Move to Meaningful Internet Systems (OTM), pages 1483-1499, 2005.

[70] Maria Karyda, Theodoros Balopoulos, Lazaros Gymnopoulos, Spyros Kokolakis, Costas Lambrinoudakis, Stefanos Gritzalis, and Stelios Dritsas. An Ontology for Secure e-government Applications. In International Conference on Availability, Reliability and Security (ARES), pages 1033-1037, 2006.

[71] Carlos Blanco, Joaquin Lasheras, Rafael Valencia-García, Eduardo Fernández-Medina, Ambrosio Toval, and Mario Piattini. A Systematic Review and Comparison of Security Ontologies. In International Conference on Availability, Reliability and Security (ARES), pages $813-820,2008$.

[72] Amina Souag, Camille Salinesi, and Isabelle Comyn-Wattiau. Ontologies for Security Requirements: A Literature Survey and Classification. In Advanced Information Systems Engineering Workshops (CAiSE), 2012.

[73] Bill Whyte and John Harrison. State of Practice in Secure Software: Experts' Views on Best Ways Ahead. Software Engineering for Secure Systems: Industrial and Research Perspectives, 2011.

[74] Acceleo. http://www.eclipse.org/acceleo/, last visited February 2013.

[75] Aamer Nadeem and Younus Javed. A Performance Comparison of Data Encryption Algorithms. In International Conference on Information and Communication Technologies (ICICT), pages 84-89, 2005.

[76] R. Stephen Preissig. Data Encryption Standard (DES) Implementation on the TMS320C6000, Application Report, 2000.

[77] Petri Selonen. A Review of UML Model Comparison Approaches. In Nordic Workshop on Model Driven Engineering, 2007.

[78] Lars Bendix and Pär Emanuelsson. Diff and Merge Support for Modelbased Development. In Workshop on Comparison and versioning of software models (CVSM), 2008. 
[79] EMF Compare; www.eclipse.org/emf/compare/, last visited April 2013.

[80] Geri Georg, Kyriakos Anastasakis, Behzad Bordbar, Siv Hilde Houmb, Indrakshi Ray, and Manachai Toahchoodee. Verification and TradeOff Analysis of Security Properties in UML System Models. IEEE, 2010 .

[81] OMAP3530. http://www.ti.com/product/omap3530, last visited February 2013.

[82] Ontology Language Manchester Syntax. http://www.w3.org/TR/ ow12-manchester-syntax/, last visited May 2013.

[83] HermiT Reasoner. http://hermit-reasoner.com, last visited september 2013.

[84] Folker den Braber, Ida Hogganvik, Soldal Lund, Ketik Stølen, and Fredrik Vraalsen. Model-Based Security Analysis in Seven Steps a Guided Tour to the CORAS Method. BT Technology Journal, 25(1):101-117, 2007.

[85] Srivaths Ravi, Anand Raghunathan, and Srimat Chakradhar. Tamper Resistance Mechanisms for Secure Embedded Systems. In International Conference on VLSI Design (VLSID). IEEE, 2004.

[86] Thomas H. Cormen, Clifford Stein, Ronald L. Rivest, and Charles E. Leiserson. Introduction to Algorithms. McGraw-Hill Higher Education, 2nd edition, 2001.

[87] Jesús M. Hermida, María Teresa Romá-Ferri, Andrés Montoyo, and Manuel Palomar. Reusing UML Class Models to Generate OWL Ontologies - A Use Case in the Pharmacotherapeutic Domain. In International Conference on Knowledge Engineering and Ontology Development (KEOD), pages 281-286. INSTICC Press, 2009.

[88] Zhuoming Xu, Yuyan Ni, Wenjie He, Lili Lin, and Qin Yan. Automatic Extraction of OWL Ontologies from UML Class Diagrams. World Wide Web, 2012.

[89] XSD Type System. http://www.w3.org/, last visited March 2013.

[90] Simple Part-whole Relations in OWL Ontologies. http://www.w3. org/2001/sw/BestPractices/OEP/SimplePartWhole/, last visited March 2013.

[91] Object Management Group. Ontology Definition Metamodel, version 1.0, May 2009. Document number: formal/2009-05-01. 
[92] Henrik Dibowski and Klaus Kabitzsch. Ontology-Based Device Descriptions and Device Repository for Building Automation Devices. EURASIP J. Embedded Syst., 2011.

[93] Texas Instruments. www.ti.com, last visited March 2013.

[94] Cryptography for C64x+-based Devices. http://www.ti.com/tool/ c64xpluscrypto, last visited January 2013.

[95] Andy Seaborne. SPARQL 1.1 Property Paths, http://www.w3. org/TR/2010/WD-sparql11-property-paths-20100126/, last visited Jan. 2013.

[96] Ray Kamal. Embedded Systems: Architecture, Programming and Design. Tata McGraw-Hill, 2009.

[97] Clemens Szyperski. Component Software: Beyond Object-Oriented Programming. Addison-Wesley Longman Publishing Co., Inc., 2002.

[98] Ivica Crnkovic. Component-based Approach for Embedded Systems. In International Conference on Software Engineering (ICSE), pages 712-713, 2005.

[99] Ed Brinksma, Geoff Coulson, Ivica Crnkovic, Andy Evans, Sébastien Gérard, Susanne Graf, Holger Hermanns, Bengt Jonsson, Anders Ravn, Philippe Schnoebelen, François Terrier, Angelika Votintseva, and Jean-Marc Jézéquel. Component-based Design and Integration Platforms. Technical report, Project IST-2001-34820. ARTIST, 2003.

[100] Ivica Crnkovic, Séverine Sentilles, Aneta Vulgarakis, and Michel Chaudron. A Classification Framework for Software Component Models. IEEE Transaction of Software Engineering, 2011.

[101] Ivica Crnkovic. Building Reliable Component-Based Software Systems. Artech House, Inc., 2002.

[102] Alberto Sangiovanni-Vincentelli and Marco Di Natale. Embedded System Design for Automotive Applications. Computer, IEEE Computer Society Press, pages 42-51, 2007.

[103] Hugh Maaskant. A Robust Component Model for Consumer Electronic Products. Dynamic and Robust Streaming in and between Connected Consumer-Electronic Devices, Springer, 2005.

[104] Petr Hošek, Tomáš Pop, Tomáš Bureš, Petr Hnětynka, and Michal Malohlava. Comparison of Component Frameworks for Real-Time Embedded Systems. In International conference on Component-Based Software Engineering (CBSE), pages 21-36. Springer-Verlag Berlin, Heidelberg, 2010. 
[105] Mehrdad Saadatmand and Thomas Leveque. Modeling Security Aspects in Distributed Real-Time Component-Based Embedded Systems. In International Conference on Information Technology : New Generations, pages 437 - 444. IEEE, 2012.

[106] Takoua Abdellatif and Nejla Rouis. Enforcing the Security of Component-based Embedded Systems with Information Flow Control. In International Conference on Wireless and Ubiquitous Systems (ICWUS). IEEE, 2010.

[107] Edsger W Dijkstra. On the Role of Scientific Thought. Selected Writings on Computing: A Personal Perspective. Springer-Verlag.

[108] Andrea Schauerhuber, Wieland Schwinger, Elisabeth Kapsammer, Werner Retschitzegger, Manuel Wimmer, and Gerti Kappel. A Survey on Aspect-Oriented Modeling Approaches. ACM Computing Surveys, 2010.

[109] Robert France, Indrakshi Ray, Geri Georg, and Sudipto Ghosh. Aspect-Oriented Approach to Early Design Modelling. IEEE Proceedings Software, 151:173-185, 2004.

[110] Makan Pourzandi, Djedjiga Mouheb, Chamseddine Talhi, Vitor Lima, Mourad Debbabi, and Lingyu Wang. Weaving Security Aspects into UML 2.0 Design Models. In Workshop on Aspect Oriented Modeling (AOM), pages 7-12, 2009.

[111] Jan Jürjens. Secure System Development with UML. Springer-Verlag, 2005.

[112] Markus Schumacher, Eduardo Fernandez-Buglioni, Duane Hybertson, Frank Buschmann, and Peter Sommerlad. Security Patterns: Integrating Security and Systems Engineering. John Wiley and Sons, 2005.

[113] Siv Hilde Houmb, Shareeful Islam, Eric Knauss, Jan Jürjens, and Kurt Schneider. Eliciting Security Requirements and Tracing Them to Design: an Integration of Common Criteria, Heuristics, and UMLsec. Requir. Eng., 15:63-93, 2010.

[114] Common Criteria for Information Technology Security Evaluation, Version 3.1., Revision 4, CCMB-2012-09-001, 2012.

[115] Torsten Lodderstedt, David Basin, and Jürgen Doser. SecureUML: A UML-Based Modeling Language for Model-Driven Security. In International Conference on The Unified Modeling Language (UML), pages 426-441. Springer-Verlag, 2002.

[116] Geri Georg, Indrakshi Ray, Kyriakos Anastasakis, Behzad Bordar, Manachai Toahchoodee, and Siv Hilde Houmb. An Aspect-Oriented 
Methodology for Designing Secure Applications. Information and Software Technology, 51(5):846-864, 2009.

[117] Geri Georg, Kyriakos Anastasakis, Behzad Bordbar, Siv Hilde Houmb, Indrakshi Ray, and Manachai Toahchoodee. Verification and TradeOff Analysis of Security Properties in UML System Models. IEEE Trans. Software Eng., 36(3):338-356, 2010.

[118] Siv Hilde Houmb, Geri Georg, Dorina Petriu, Behzad Bordbar, Indrakshi Ray, Kyriakos Anastasakis, and Robert France. Balancing Security and Performance Properties During System Architectural Design. Software Engineering for Secure Systems: Industrial and Research Perspectives, 2011.

[119] Holger Schmidt, Denis Hatebur, and Maritta Heisel. A Pattern-Based Method to Develop Secure Software. Software Engineering for Secure Systems: Industrial and Research Perspectives, 2011.

[120] Brahim Hamid, Sigrid Gürgens, Christophe Jouvray, and Nicolas Desnos. Enforcing S\&D Pattern Design in RCES with Modeling and Formal Approaches. In ACM/IEEE International Conference on Model Driven Engineering Languages and Systems (MoDELS), pages 319-333, 2011.

[121] Gabriel Pedroza, Ludovic Apvrille, and Daniel Knorreck. AVATAR: A SysML Environment for the Formal Verification of Safety and Security Properties. In IEEE International Conference on New Technologies of Distributed Systems (NOTERE), pages 1 - 10, 2011.

[122] Anton V. Uzunov, Eduardo B. Fernandez, and Katrina Falkner. Engineering Security into Distributed Systems: A Survey of Methodologies. Journal of Universal Computer Science, 18(20):2920-3006, 2012.

[123] Jose Fran. Ruiz, Rajesh Harjani, Antonio Mana, Vasily Desnitsky, Igor Kotenko, and Andrey Chechulin. A Methodology for the Analysis and Modeling of Security Threats and Attacks for Systems of Embedded Components. In Euromicro International Conference on Parallel, Distributed and Network-Based Processing (PDP), pages 261-268. IEEE Computer Society, 2012.

[124] Yomna Ali, Sherif El-Kassas, and Mohy Mahmoud. A Rigorous Methodology for Security Architecture Modeling and Verification. In International Conference on System Sciences (HICSS), pages 1-10, 2009 .

[125] Brahim Hamid, Nicolas Desnos, Cyril Grepet, and Christophe Jouvray. Model-Based Security and Dependability Patterns in RCES - the TERESA Approach. In International Workshop on Security and Dependability for Resource Constrained Embedded Systems (SED4RCES). ACM, 2010. 
[126] Matthew Eby, Jan Werner, Gabor Karsai, and Akos Ledeczi. Integrating Security Modeling into Embedded System Design. IEEE International Conference and Workshops on the Engineering of ComputerBased Systems (ECBS), pages 221 - 228, March 2007.

[127] Eduardo B. Fernandez, Hironori Washizaki, Nobukazu Yoshioka, Atsuto Kubo, and Yoshiaki Fukazawa. Classifying Security Patterns. In Asia-Pacific Web Conference on Progress in $W W W$ Research and Development (APWeb), pages 342-347, 2008.

[128] Michael VanHilst, Eduardo B. Fernández, and Fabrício A. Braz. A Multi-Dimensional Classification for Users of Security Patterns. Journal of Research and Practice in Information Technology, Australian Computer Society Inc., 20(1), 2008.

[129] Munawar Hafiz, Paul Adamczyk, and Ralph E. Johnson. Organizing Security Patterns. In 4, editor, Software, IEEE, volume 24, pages 52-60, 2007.

[130] Hironori Washizaki, Eduardo B. Fernandez, Katsuhisa Maruyama, Atsuto Kubo, and Nobukazu Yoshioka. Improving the Classification of Security Patterns. In International Workshop on Database and Expert Systems Application (DEXA), pages 165-170, 2009.

[131] Alberts Christopher, Sandra Behrens, Richard Pethia, and William Wilson. Operationally Critical Threat, Asset, and Vulnerability Evaluation (OCTAVE) Framework, Version 1.0. Technical report, Technical Report CMU/SEI-99-TR-017. ESC-TR-99-017, Carnegie Mellon. Software Engineering Institute, 1999.

[132] CCTA Risk Analysis and Management Method. http://www.cramm. com, last visited october 2013.

[133] Dragan Gasevic, Dragan Djuric, and Vladan Devedzic. Model Driven Engineering and Ontology Development. Springer Publishing Company, 2009.

[134] Phil Tetlow, Jeff Z. Pan, Daniel Oberle, Evan Wallace, Michael Uschold, and Elisa Kendall. Ontology Driven Architectures and Potential Uses of the Semantic Web in Systems and Software Engineering. http://www.w3.org/2001/sw/BestPractices/SE/ODA/, last visited may 2013.

[135] Tobias Walter, Fernando Silva Parreiras, and Steffen Staab. An Ontology-based Framework for Domain-specific Modeling. In Softw. Syst. Model. Springer-Verlag, 2012. 
[136] Jianjun Yi, Ying Cheng, and Chunhua Gu. A Reconfigurable Design Method of Embedded Control System based on Ontology. In International Conference on E-Product E-Service and E-Entertainment (ICEEE), pages 1 - 4. IEEE, 2010.

[137] Dennis Wagelaar. Platform Ontologies for the Model-Driven Architecture. PhD thesis, 2010.

[138] Bedir Tekinerdoğan, Sevcan Bilir, and Cem Abatlevi. Integrating Platform Selection Rules in the Model Driven Architecture Approach. In European conference on Model Driven Architecture: foundations and Applications (MDAFA), pages 159-173. Springer-Verlag, 2003.

[139] Thomas Robert and Vincent Perrier. CoFluent Methodology for UML. A CoFluent Design, White Paper, 2010.

[140] Éric Piel, Samy Meftali, Jean luc Dekeyser, Rabie Ben Atitallah, Smaïl Niar, Anne Etien, and Pierre Boulet. Gaspard2: from MARTE to SystemC Simulation. In Workshop on Modeling and Analysis of RealTime and Embedded Systems (at DATE), 2008.

[141] Object Management Group. Systems Modeling Language: verstion 1.3, 2012.

[142] Murray Woodside, Dorina C. Petriu, Dorin B. Petriu, Jing Xu, Tauseef Israr, Geri Georg, Robert France, James M. Bieman, Siv Hilde Houmb, and Jan Jürjens. Performance Analysis of Security Aspects by Weaving Scenarios Extracted from UML models. Journal of Systems and Software, Elsevier Science Inc., 2009.

[143] Murray Woodside, Dorina C. Petriu, Dorin B. Petriu, Hui Shen, Toqeer Israr, and Jose Merseguer. Performance by Unified Model Analysis (PUMA). In ACM Workhsop on Software and Performance (WOSP), pages 1-12. ACM, 2005.

[144] Marco A. Wehrmeister, Edison P. Freitas, Carlos E. Pereira, and Franz Rammig. GenERTiCA: A Tool for Code Generation and Aspects Weaving. In IEEE International Symposium on Object Oriented RealTime Distributed Computing (ISORC), pages 234 - 238, 2008.

[145] Egor Bondarev, Michel Chaudron, and Peter H. N. de With. CARAT: a Toolkit for Design and Performance Analysis of Component-Based Embedded Systems. In The conference on Design, automation and test in Europe (DATE), pages 1024-1029. EDA Consortium, 2007.

[146] Federico Ciccozzi, Antonio Cicchetti, and Mikael Sjödin. Round-trip Support for Extra-functional Property Management in Model-driven Engineering of Embedded Systems. Information and Software Technology, Elsevier, pages 1085-1100, 2012. 
[147] ARTEMIS-JU-216682, CHESS. http://chess-project.ning.com/, last visited March 2013. 

Department of Computer and Information Science

Linköpings universitet

\section{Licentiate Theses}

\section{Linköpings Studies in Science and Technology Faculty of Arts and Sciences}

No 17 Vojin Plavsic: Interleaved Processing of Non-Numerical Data Stored on a Cyclic Memory. (Available at: FOA, Box 1165, S-581 11 Linköping, Sweden. FOA Report B30062E)

No 28 Arne Jönsson, Mikael Patel: An Interactive Flowcharting Technique for Communicating and Realizing Algorithms, 1984.

No 29 Johnny Eckerland: Retargeting of an Incremental Code Generator, 1984.

No 48

No 52

No 60

No 71

No 72

No 73

No 74

No 104

Henrik Nordin: On the Use of Typical Cases for Knowledge-Based Consultation and Teaching, 1985.

Zebo Peng: Steps Towards the Formalization of Designing VLSI Systems, 1985.

Johan Fagerström: Simulation and Evaluation of Architecture based on Asynchronous Processes, 1985.

Jalal Maleki: ICONStraint, A Dependency Directed Constraint Maintenance System, 1987.

Tony Larsson: On the Specification and Verification of VLSI Systems, 1986.

Ola Strömfors: A Structure Editor for Documents and Programs, 1986.

Christos Levcopoulos: New Results about the Approximation Behavior of the Greedy Triangulation, 1986.

No 108

Shamsul I. Chowdhury: Statistical Expert Systems - a Special Application Area for Knowledge-Based Computer Methodology, 1987.

No 111

No 113

No 118

Rober Bilos: Incremental Scanning and Token-Based Editing, 1987.

Hans Block: SPORT-SORT Sorting Algorithms and Sport Tournaments, 1987.

Ralph Rönnquist: Network and Lattice Based Approaches to the Representation of Knowledge, 1987.

Mariam Kamkar, Nahid Shahmehri: Affect-Chaining in Program Flow Analysis Applied to Queries of Programs, 1987.

No 126 Dan Strömberg: Transfer and Distribution of Application Programs, 1987.

No 127

No 139

No 140

No 146

No 150

No 165

No 166

No 174

No 177

No 181

No 184

No 187

No 189

No 196

No 197

No 203

No 212

No 230

No 237

No 250

No 253

No 260

No 283

Kristian Sandahl: Case Studies in Knowledge Acquisition, Migration and User Acceptance of Expert Systems, 1987.

Christer Bäckström: Reasoning about Interdependent Actions, 1988.

Mats Wirén: On Control Strategies and Incrementality in Unification-Based Chart Parsing, 1988.

Johan Hultman: A Software System for Defining and Controlling Actions in a Mechanical System, 1988.

Tim Hansen: Diagnosing Faults using Knowledge about Malfunctioning Behavior, 1988.

Jonas Löwgren: Supporting Design and Management of Expert System User Interfaces, 1989.

Ola Petersson: On Adaptive Sorting in Sequential and Parallel Models, 1989.

Yngve Larsson: Dynamic Configuration in a Distributed Environment, 1989.

Peter Åberg: Design of a Multiple View Presentation and Interaction Manager, 1989.

Henrik Eriksson: A Study in Domain-Oriented Tool Support for Knowledge Acquisition, 1989.

Ivan Rankin: The Deep Generation of Text in Expert Critiquing Systems, 1989.

Simin Nadjm-Tehrani: Contributions to the Declarative Approach to Debugging Prolog Programs, 1989.

Magnus Merkel: Temporal Information in Natural Language, 1989.

Ulf Nilsson: A Systematic Approach to Abstract Interpretation of Logic Programs, 1989.

Staffan Bonnier: Horn Clause Logic with External Procedures: Towards a Theoretical Framework, 1989.

Christer Hansson: A Prototype System for Logical Reasoning about Time and Action, 1990.

Björn Fjellborg: An Approach to Extraction of Pipeline Structures for VLSI High-Level Synthesis, 1990.

Patrick Doherty: A Three-Valued Approach to Non-Monotonic Reasoning, 1990.

Tomas Sokolnicki: Coaching Partial Plans: An Approach to Knowledge-Based Tutoring, 1990.

Lars Strömberg: Postmortem Debugging of Distributed Systems, 1990.

Torbjörn Näslund: SLDFA-Resolution - Computing Answers for Negative Queries, 1990.

Peter D. Holmes: Using Connectivity Graphs to Support Map-Related Reasoning, 1991.

Olof Johansson: Improving Implementation of Graphical User Interfaces for Object-Oriented Knowledge- Bases, 1991.

No 298 Rolf G Larsson: Aktivitetsbaserad kalkylering i ett nytt ekonomisystem, 1991.

No 318 Lena Srömbäck: Studies in Extended Unification-Based Formalism for Linguistic Description: An Algorithm for Feature Structures with Disjunction and a Proposal for Flexible Systems, 1992.

No 319 Mikael Pettersson: DML-A Language and System for the Generation of Efficient Compilers from Denotational Specification, 1992.

No 326 Andreas Kågedal: Logic Programming with External Procedures: an Implementation, 1992.

No 328

No 333

No 335

No 348

No 352

Patrick Lambrix: Aspects of Version Management of Composite Objects, 1992.

Xinli Gu: Testability Analysis and Improvement in High-Level Synthesis Systems, 1992.

Torbjörn Näslund: On the Role of Evaluations in Iterative Development of Managerial Support Systems, 1992.

Ulf Cederling: Industrial Software Development - a Case Study, 1992.

No 371 Mehran Noghabai: Evaluation of Strategic Investments in Information Technology, 1993.

No 378

Magnus Morin: Predictable Cyclic Computations in Autonomous Systems: A Computational Model and Implementation, 1992.

Mats Larsson: A Transformational Approach to Formal Digital System Design, 1993. 

Peter Carlsson: Separation av företagsledning och finansiering - fallstudier av företagsledarutköp ur ett agentteoretiskt perspektiv, 1994.

FHS 4/94 Karin Pettersson: Informationssystemstrukturering, ansvarsfördelning och användarinflytande - En komparativ studie med utgångspunkt i två informationssystemstrategier, 1994.

No 441 Lars Poignant: Informationsteknologi och företagsetablering - Effekter på produktivitet och region, 1994.

No 446 Gustav Fahl: Object Views of Relational Data in Multidatabase Systems, 1994.

No 450

No 451 Pär Carlshamre: A Collaborative Approach to Usability Engineering: Technical Communicators and System Developers in Usability-Oriented Systems Development, 1994.

FHS 5/94 Stefan Cronholm: Varför CASE-verktyg i systemutveckling? - En motiv- och konsekvensstudie avseende arbetssätt och arbetsformer, 1994.

No 462

No 463

No 464

No 469

No 473

No 475

No 476

No 478

FHS 7/95

Mikael Lindvall: A Study of Traceability in Object-Oriented Systems Development, 1994.

Fredrik Nilsson: Strategi och ekonomisk styrning - En studie av Sandviks förvärv av Bahco Verktyg, 1994.

Hans Olsén: Collage Induction: Proving Properties of Logic Programs by Program Synthesis, 1994.

Lars Karlsson: Specification and Synthesis of Plans Using the Features and Fluents Framework, 1995.

Ulf Söderman: On Conceptual Modelling of Mode Switching Systems, 1995.

Choong-ho Yi: Reasoning about Concurrent Actions in the Trajectory Semantics, 1995.

Bo Lagerström: Successiv resultatavräkning av pågående arbeten. - Fallstudier i tre byggföretag, 1995.

Peter Jonsson: Complexity of State-Variable Planning under Structural Restrictions, 1995.

Anders Avdic: Arbetsintegrerad systemutveckling med kalkylprogram, 1995.

No 482 Eva L Ragnemalm: Towards Student Modelling through Collaborative Dialogue with a Learning Companion, 1995.

No 488 Eva Toller: Contributions to Parallel Multiparadigm Languages: Combining Object-Oriented and Rule-Based Programming, 1995.

No 489 Erik Stoy: A Petri Net Based Unified Representation for Hardware/Software Co-Design, 1995.

No 497

No 498

Johan Herber: Environment Support for Building Structured Mathematical Models, 1995.

No 503 Hee-Cheol Kim: Prediction and Postdiction under Uncertainty, 1995.

FHS 8/95 Dan Fristedt: Metoder i användning - mot förbättring av systemutveckling genom situationell metodkunskap och metodanalys, 1995.

FHS 9/95 Malin Bergvall: Systemförvaltning i praktiken - en kvalitativ studie avseende centrala begrepp, aktiviteter och ansvarsroller, 1995.

No 513 Joachim Karlsson: Towards a Strategy for Software Requirements Selection, 1995.

No 517

No 518

No 522

No 538

No 545

Jakob Axelsson: Schedulability-Driven Partitioning of Heterogeneous Real-Time Systems, 1995.

Göran Forslund: Toward Cooperative Advice-Giving Systems: The Expert Systems Experience, 1995.

Jörgen Andersson: Bilder av småföretagares ekonomistyrning, 1995.

Staffan Flodin: Efficient Management of Object-Oriented Queries with Late Binding, 1996.

Vadim Engelson: An Approach to Automatic Construction of Graphical User Interfaces for Applications in Scientific Computing, 1996.

No 546

FiF-a $1 / 96$

No 549

Magnus Werner : Multidatabase Integration using Polymorphic Queries and Views, 1996.

No 550

Mikael Lind: Affärsprocessinriktad förändringsanalys - utveckling och tillämpning av synsätt och metod, 1996.

Jonas Hallberg: High-Level Synthesis under Local Timing Constraints, 1996.

No 557

No 558

No 561

No 563

Kristina Larsen: Förutsättningar och begränsningar för arbete på distans - erfarenheter från fyra svenska företag. 1996.

Mikael Johansson: Quality Functions for Requirements Engineering Methods, 1996.

Patrik Nordling: The Simulation of Rolling Bearing Dynamics on Parallel Computers, 1996.

Anders Ekman: Exploration of Polygonal Environments, 1996.

Niclas Andersson: Compilation of Mathematical Models to Parallel Code, 1996. 
Johan Jenvald: Simulation and Data Collection in Battle Training, 1996.

Jörgen Lindström: Chefers användning av kommunikationsteknik, 1996.

No 589 Esa Falkenroth: Data Management in Control Applications - A Proposal Based on Active Database Systems, 1996.

No 591

No 595

No 597

No 598

No 599

No 607

No 609

FiF-a 4

FiF-a 6

No 615

No 623

No 626

No 627

No 629

No 631

No 639

No 640

No 643

No 653

FiF-a 13

No 674

No 676

No 668

No 675

FiF-a 14

No 695

No 700

FiF-a 16

No 712

No 719

No 723

No 725

No 730

No 731

No 733

No 734

FiF-a 21

FiF-a 22

No 737

No 738

FiF-a 25

No 742

No 748

No 751

No 752

No 753

Niclas Wahllöf: A Default Extension to Description Logics and its Applications, 1996.

Annika Larsson: Ekonomisk Styrning och Organisatorisk Passion - ett interaktivt perspektiv, 1997.

Ling Lin: A Value-based Indexing Technique for Time Sequences, 1997.

Rego Granlund: $C^{3}$ Fire - A Microworld Supporting Emergency Management Training, 1997.

Peter Ingels: A Robust Text Processing Technique Applied to Lexical Error Recovery, 1997.

Per-Arne Persson: Toward a Grounded Theory for Support of Command and Control in Military Coalitions, 1997.

Jonas S Karlsson: A Scalable Data Structure for a Parallel Data Server, 1997.

Carita Åbom: Videomötesteknik i olika affärssituationer - möjligheter och hinder, 1997.

Tommy Wedlund: Att skapa en företagsanpassad systemutvecklingsmodell - genom rekonstruktion, värdering och vidareutveckling i T50-bolag inom ABB, 1997.

Silvia Coradeschi: A Decision-Mechanism for Reactive and Coordinated Agents, 1997.

Jan Ollinen: Det flexibla kontorets utveckling på Digital - Ett stöd för multiflex? 1997.

David Byers: Towards Estimating Software Testability Using Static Analysis, 1997.

Fredrik Eklund: Declarative Error Diagnosis of GAPLog Programs, 1997.

Gunilla Ivefors: Krigsspel och Informationsteknik inför en oförutsägbar framtid, 1997.

Jens-Olof Lindh: Analysing Traffic Safety from a Case-Based Reasoning Perspective, 1997

Jukka Mäki-Turja:. Smalltalk - a suitable Real-Time Language, 1997.

Juha Takkinen: CAFE: Towards a Conceptual Model for Information Management in Electronic Mail, 1997.

Man Lin: Formal Analysis of Reactive Rule-based Programs, 1997.

Mats Gustafsson: Bringing Role-Based Access Control to Distributed Systems, 1997.

Boris Karlsson: Metodanalys för förståelse och utveckling av systemutvecklingsverksamhet. Analys och värdering av systemutvecklingsmodeller och dess användning, 1997.

Marcus Bjäreland: Two Aspects of Automating Logics of Action and Change - Regression and Tractability, 1998.

Jan Håkegård: Hierarchical Test Architecture and Board-Level Test Controller Synthesis, 1998.

Per-Ove Zetterlund: Normering av svensk redovisning - En studie av tillkomsten av Redovisningsrådets rekommendation om koncernredovisning (RR01:91), 1998.

Jimmy Tjäder: Projektledaren \& planen - en studie av projektledning i tre installations- och systemutvecklingsprojekt, 1998.

1998.

Tim Heyer: COMPASS: Introduction of Formal Methods in Code Development and Inspection, 1998.

Patrik Hägglund: Programming Languages for Computer Algebra, 1998.

Marie-Therese Christiansson: Inter-organisatorisk verksamhetsutveckling - metoder som stöd vid utveckling av partnerskap och informationssystem, 1998.

Christina Wennestam: Information om immateriella resurser. Investeringar i forskning och utveckling samt i personal inom skogsindustrin, 1998.

Joakim Gustafsson: Extending Temporal Action Logic for Ramification and Concurrency, 1998.

Henrik André-Jönsson: Indexing time-series data using text indexing methods, 1999.

Erik Larsson: High-Level Testability Analysis and Enhancement Techniques, 1998.

Carl-Johan Westin: Informationsförsörjning: en fråga om ansvar - aktiviteter och uppdrag i fem stora svenska organisationers operativa informationsförsörjning, 1998.

Åse Jansson: Miljöhänsyn - en del i företags styrning, 1998.

Thomas Padron-McCarthy: Performance-Polymorphic Declarative Queries, 1998.

Anders Bäckström: Värdeskapande kreditgivning - Kreditriskhantering ur ett agentteoretiskt perspektiv, 1998.

Ulf Seigerroth: Integration av förändringsmetoder - en modell för välgrundad metodintegration, 1999.

Fredrik Öberg: Object-Oriented Frameworks - A New Strategy for Case Tool Development, 1998.

Jonas Mellin: Predictable Event Monitoring, 1998.

Joakim Eriksson: Specifying and Managing Rules in an Active Real-Time Database System, 1998.

Bengt E W Andersson: Samverkande informationssystem mellan aktörer i offentliga åtaganden - En teori om aktörsarenor i samverkan om utbyte av information, 1998.

Pawel Pietrzak: Static Incorrectness Diagnosis of CLP (FD), 1999.

Tobias Ritzau: Real-Time Reference Counting in RT-Java, 1999.

Anders Ferntoft: Elektronisk affärskommunikation - kontaktkostnader och kontaktprocesser mellan kunder och leverantörer på producentmarknader, 1999.

Jo Skåmedal: Arbete på distans och arbetsformens påverkan på resor och resmönster, 1999.

Johan Alvehus: Mötets metaforer. En studie av berättelser om möten, 1999. 
Magnus Lindahl: Bankens villkor i låneavtal vid kreditgivning till högt belånade företagsförvärv: En studie ur ett agentteoretiskt perspektiv, 2000.

No 766

No 769

No 775

FiF-a 30

No 787

No 788

No 790

No 791

No 800

No 807

No 809

FiF-a 32

No 808

No 820

No 823

No 832

FiF-a 34

No 842

No 844

FiF-a 37

FiF-a 40

FiF-a 41

No. 854

No 863

No 881

No 882

No 890

FiF-a 47

No 894

No 906

No 917

No 916

FiF-a-49

FiF-a-51

No 919

No 915

No 931

No 933

No 938

No 942

No 956

FiF-a 58

No 964

No 973

No 958

FiF-a 61

No 985

No 982

No 989

No 990

No 991

Martin V. Howard: Designing dynamic visualizations of temporal data, 1999.

Jesper Andersson: Towards Reactive Software Architectures, 1999.

Anders Henriksson: Unique kernel diagnosis, 1999.

Pär J. Ågerfalk: Pragmatization of Information Systems - A Theoretical and Methodological Outline, 1999.

Charlotte Björkegren: Learning for the next project - Bearers and barriers in knowledge transfer within an organisation, 1999.

2000.

Erik Berglund: Use-Oriented Documentation in Software Development, 1999.

Klas Gäre: Verksamhetsförändringar i samband med IS-införande, 1999.

Anders Subotic: Software Quality Inspection, 1999.

Svein Bergum: Managerial communication in telework, 2000.

Flavius Gruian: Energy-Aware Design of Digital Systems, 2000.

Karin Hedström: Kunskapsanvändning och kunskapsutveckling hos verksamhetskonsulter - Erfarenheter från ett FOU-samarbete, 2000.

Linda Askenäs: Affärssystemet - En studie om teknikens aktiva och passiva roll i en organisation, 2000.

Jean Paul Meynard: Control of industrial robots through high-level task programming, 2000.

Lars Hult: Publika Gränsytor - ett designexempel, 2000.

Paul Pop: Scheduling and Communication Synthesis for Distributed Real-Time Systems, 2000.

Göran Hultgren: Nätverksinriktad Förändringsanalys - perspektiv och metoder som stöd för förståelse och utveckling av affärsrelationer och informationssystem, 2000.

Magnus Kald: The role of management control systems in strategic business units, 2000.

Mikael Cäker: Vad kostar kunden? Modeller för intern redovisning, 2000.

Ewa Braf: Organisationers kunskapsverksamheter - en kritisk studie av "knowledge management", 2000.

Henrik Lindberg: Webbaserade affärsprocesser - Möjligheter och begränsningar, 2000.

Benneth Christiansson: Att komponentbasera informationssystem - Vad säger teori och praktik?, 2000.

Ola Pettersson: Deliberation in a Mobile Robot, 2000.

Dan Lawesson: Towards Behavioral Model Fault Isolation for Object Oriented Control Systems, 2000.

Johan Moe: Execution Tracing of Large Distributed Systems, 2001.

Yuxiao Zhao: XML-based Frameworks for Internet Commerce and an Implementation of B2B e-procurement, 2001.

Annika Flycht-Eriksson: Domain Knowledge Management in Information-providing Dialogue systems, 2001.

Per-Arne Segerkvist: Webbaserade imaginära organisationers samverkansformer: Informationssystemarkitektur och aktörssamverkan som förutsättningar för affärsprocesser, 2001.

Stefan Svarén: Styrning av investeringar i divisionaliserade företag - Ett koncernperspektiv, 2001.

Lin Han: Secure and Scalable E-Service Software Delivery, 2001.

Emma Hansson: Optionsprogram för anställda - en studie av svenska börsföretag, 2001.

Susanne Odar: IT som stöd för strategiska beslut, en studie av datorimplementerade modeller av verksamhet som stöd för beslut om anskaffning av JAS 1982, 2002.

Stefan Holgersson: IT-system och filtrering av verksamhetskunskap - kvalitetsproblem vid analyser och beslutsfattande som bygger på uppgifter hämtade från polisens IT-system, 2001.

Per Oscarsson: Informationssäkerhet i verksamheter - begrepp och modeller som stöd för förståelse av informationssäkerhet och dess hantering, 2001.

Luis Alejandro Cortes: A Petri Net Based Modeling and Verification Technique for Real-Time Embedded Systems, 2001.

Niklas Sandell: Redovisning i skuggan av en bankkris - Värdering av fastigheter. 2001.

Fredrik Elg: Ett dynamiskt perspektiv på individuella skillnader av heuristisk kompetens, intelligens, mentala modeller, mål och konfidens i kontroll av mikrovärlden Moro, 2002.

Peter Aronsson: Automatic Parallelization of Simulation Code from Equation Based Simulation Languages, 2002.

Bourhane Kadmiry: Fuzzy Control of Unmanned Helicopter, 2002.

Patrik Haslum: Prediction as a Knowledge Representation Problem: A Case Study in Model Design, 2002.

Robert Sevenius: On the instruments of governance - A law \& economics study of capital instruments in limited liability companies, 2002.

Johan Petersson: Lokala elektroniska marknadsplatser - informationssystem för platsbundna affärer, 2002.

Peter Bunus: Debugging and Structural Analysis of Declarative Equation-Based Languages, 2002.

Gert Jervan: High-Level Test Generation and Built-In Self-Test Techniques for Digital Systems, 2002.

Fredrika Berglund: Management Control and Strategy - a Case Study of Pharmaceutical Drug Development, 2002.

Fredrik Karlsson: Meta-Method for Method Configuration - A Rational Unified Process Case, 2002.

Sorin Manolache: Schedulability Analysis of Real-Time Systems with Stochastic Task Execution Times, 2002.

Diana Szentiványi: Performance and Availability Trade-offs in Fault-Tolerant Middleware, 2002.

Iakov Nakhimovski: Modeling and Simulation of Contacting Flexible Bodies in Multibody Systems, 2002.

Levon Saldamli: PDEModelica - Towards a High-Level Language for Modeling with Partial Differential Equations, 2002.

Almut Herzog: Secure Execution Environment for Java Electronic Services, 2002. 
Jon Edvardsson: Contributions to Program- and Specification-based Test Data Generation, 2002.

No 1001 Andrzej Bednarski: A Dynamic Programming Approach to Optimal Retargetable Code Generation for Irregular Architectures, 2002.

No 988 Mattias Arvola: Good to use! : Use quality of multi-user applications in the home, 2003.

FiF-a 62 Lennart Ljung: Utveckling av en projektivitetsmodell - om organisationers förmåga att tillämpa projektarbetsformen, 2003.

No 1003 Pernilla Qvarfordt: User experience of spoken feedback in multimodal interaction, 2003.

No 1005 Alexander Siemers: Visualization of Dynamic Multibody Simulation With Special Reference to Contacts, 2003.

No 1008 Jens Gustavsson: Towards Unanticipated Runtime Software Evolution, 2003.

No 1010 Calin Curescu: Adaptive QoS-aware Resource Allocation for Wireless Networks, 2003.

No 1015

No 1018

No 1022

Anna Andersson: Management Information Systems in Process-oriented Healthcare Organisations, 2003.

Björn Johansson: Feedforward Control in Dynamic Situations, 2003.

Traian Pop: Scheduling and Optimisation of Heterogeneous Time/Event-Triggered Distributed Embedded Systems, 2003.

FiF-a 65 Britt-Marie Johansson: Kundkommunikation på distans - en studie om kommunikationsmediets betydelse i affärstransaktioner, 2003.

No 1024 Aleksandra Tešanovic: Towards Aspectual Component-Based Real-Time System Development, 2003.

No 1034 Arja Vainio-Larsson: Designing for Use in a Future Context - Five Case Studies in Retrospect, 2003.

No 1033 Peter Nilsson: Svenska bankers redovisningsval vid reservering för befarade kreditförluster - En studie vid införandet av nya redovisningsregler, 2003.

FiF-a 69 Fredrik Ericsson: Information Technology for Learning and Acquiring of Work Knowledge, 2003.

No 1049 Marcus Comstedt: Towards Fine-Grained Binary Composition through Link Time Weaving, 2003.

No 1052 Åsa Hedenskog: Increasing the Automation of Radio Network Control, 2003.

No 1054 Claudiu Duma: Security and Efficiency Tradeoffs in Multicast Group Key Management, 2003.

FiF-a $71 \quad$ Emma Eliason: Effektanalys av IT-systems handlingsutrymme, 2003.

No 1055 Carl Cederberg: Experiments in Indirect Fault Injection with Open Source and Industrial Software, 2003.

No 1058 Daniel Karlsson: Towards Formal Verification in a Component-based Reuse Methodology, 2003.

FiF-a 73 Anders Hjalmarsson: Att etablera och vidmakthålla förbättringsverksamhet - behovet av koordination och interaktion vid förändring av systemutvecklingsverksamheter, 2004.

No 1079 Pontus Johansson: Design and Development of Recommender Dialogue Systems, 2004.

No 1084 Charlotte Stoltz: Calling for Call Centres - A Study of Call Centre Locations in a Swedish Rural Region, 2004.

FiF-a 74 Björn Johansson: Deciding on Using Application Service Provision in SMEs, 2004.

No 1094 Genevieve Gorrell: Language Modelling and Error Handling in Spoken Dialogue Systems, 2004.

No 1095 Ulf Johansson: Rule Extraction - the Key to Accurate and Comprehensible Data Mining Models, 2004.

No 1099 Sonia Sangari: Computational Models of Some Communicative Head Movements, 2004.

No 1110 Hans Nässla: Intra-Family Information Flow and Prospects for Communication Systems, 2004.

No 1116 Henrik Sällberg: On the value of customer loyalty programs - A study of point programs and switching costs, 2004.

FiF-a 77 Ulf Larsson: Designarbete i dialog - karaktärisering av interaktionen mellan användare och utvecklare i en systemutvecklingsprocess, 2004.

No 1126 Andreas Borg: Contribution to Management and Validation of Non-Functional Requirements, 2004.

No 1127 Per-Ola Kristensson: Large Vocabulary Shorthand Writing on Stylus Keyboard, 2004.

No 1132 Pär-Anders Albinsson: Interacting with Command and Control Systems: Tools for Operators and Designers, 2004.

No $1130 \quad$ Ioan Chisalita: Safety-Oriented Communication in Mobile Networks for Vehicles, 2004.

No 1138 Thomas Gustafsson: Maintaining Data Consistency in Embedded Databases for Vehicular Systems, 2004.

No 1149 Vaida Jakoniené: A Study in Integrating Multiple Biological Data Sources, 2005.

No 1156 Abdil Rashid Mohamed: High-Level Techniques for Built-In Self-Test Resources Optimization, 2005.

No 1162 Adrian Pop: Contributions to Meta-Modeling Tools and Methods, 2005.

No 1165 Fidel Vascós Palacios: On the information exchange between physicians and social insurance officers in the sick leave process: an Activity Theoretical perspective, 2005.

FiF-a 84 Jenny Lagsten: Verksamhetsutvecklande utvärdering i informationssystemprojekt, 2005.

No 1166 Emma Larsdotter Nilsson: Modeling, Simulation, and Visualization of Metabolic Pathways Using Modelica, 2005.

No 1167 Christina Keller: Virtual Learning Environments in higher education. A study of students' acceptance of educational technology, 2005.

No 1168 Cécile Åberg: Integration of organizational workflows and the Semantic Web, 2005.

FiF-a 85 Anders Forsman: Standardisering som grund för informationssamverkan och IT-tjänster - En fallstudie baserad på trafikinformationstjänsten RDS-TMC, 2005.

No 1171 Yu-Hsing Huang: A systemic traffic accident model, 2005.

FiF-a 86 Jan Olausson: Att modellera uppdrag - grunder för förståelse av processinriktade informationssystem i transaktionsintensiva verksamheter, 2005.

No 1172 Petter Ahlström: Affärsstrategier för seniorbostadsmarknaden, 2005.

No 1183 Mathias Cöster: Beyond IT and Productivity - How Digitization Transformed the Graphic Industry, 2005.

No 1184 Åsa Horzella: Beyond IT and Productivity - Effects of Digitized Information Flows in Grocery Distribution, 2005.

No 1185 Maria Kollberg: Beyond IT and Productivity - Effects of Digitized Information Flows in the Logging Industry, 2005.

No 1190 David Dinka: Role and Identity - Experience of technology in professional settings, 2005. 
Andreas Hansson: Increasing the Storage Capacity of Recursive Auto-associative Memory by Segmenting Data, 2005 .

No 1192 Nicklas Bergfeldt: Towards Detached Communication for Robot Cooperation, 2005

No 1194 Dennis Maciuszek: Towards Dependable Virtual Companions for Later Life, 2005.

No 1204 Beatrice Alenljung: Decision-making in the Requirements Engineering Process: A Human-centered Approach, 2005.

No 1206 Anders Larsson: System-on-Chip Test Scheduling and Test Infrastructure Design, 2005.

No 1207 John Wilander: Policy and Implementation Assurance for Software Security, 2005.

No 1209 Andreas Käll: Översättningar av en managementmodell - En studie av införandet av Balanced Scorecard i ett landsting, 2005.

No 1225 He Tan: Aligning and Merging Biomedical Ontologies, 2006.

No 1228 Artur Wilk: Descriptive Types for XML Query Language Xcerpt, 2006.

No 1229 Per Olof Pettersson: Sampling-based Path Planning for an Autonomous Helicopter, 2006.

No 1231

Kalle Burbeck: Adaptive Real-time Anomaly Detection for Safeguarding Critical Networks, 2006.

No 1233 Daniela Mihailescu: Implementation Methodology in Action: A Study of an Enterprise Systems Implementation Methodology, 2006.

No 1244 Jörgen Skågeby: Public and Non-public gifting on the Internet, 2006.

No 1248 Karolina Eliasson: The Use of Case-Based Reasoning in a Human-Robot Dialog System, 2006.

No 1263 Misook Park-Westman: Managing Competence Development Programs in a Cross-Cultural Organisation - What are the Barriers and Enablers, 2006.

FiF-a $90 \quad$ Amra Halilovic: Ett praktikperspektiv på hantering av mjukvarukomponenter, 2006.

No 1272 Raquel Flodström: A Framework for the Strategic Management of Information Technology, 2006.

No 1277 Viacheslav Izosimov: Scheduling and Optimization of Fault-Tolerant Embedded Systems, 2006.

No 1283 Håkan Hasewinkel: A Blueprint for Using Commercial Games off the Shelf in Defence Training, Education and Research Simulations, 2006.

FiF-a 91 Hanna Broberg: Verksamhetsanpassade IT-stöd - Designteori och metod, 2006.

No 1286 Robert Kaminski: Towards an XML Document Restructuring Framework, 2006.

No 1293 Jiri Trnka: Prerequisites for data sharing in emergency management, 2007.

No 1302 Björn Hägglund: A Framework for Designing Constraint Stores, 2007.

No 1303 Daniel Andreasson: Slack-Time Aware Dynamic Routing Schemes for On-Chip Networks, 2007.

No 1305 Magnus Ingmarsson: Modelling User Tasks and Intentions for Service Discovery in Ubiquitous Computing, 2007.

No 1306 Gustaf Svedjemo: Ontology as Conceptual Schema when Modelling Historical Maps for Database Storage, 2007.

No 1307 Gianpaolo Conte: Navigation Functionalities for an Autonomous UAV Helicopter, 2007.

No 1309 Ola Leifler: User-Centric Critiquing in Command and Control: The DKExpert and ComPlan Approaches, 2007.

No 1312 Henrik Svensson: Embodied simulation as off-line representation, 2007.

No 1313 Zhiyuan He: System-on-Chip Test Scheduling with Defect-Probability and Temperature Considerations, 2007.

No 1317 Jonas Elmqvist: Components, Safety Interfaces and Compositional Analysis, 2007.

No 1320 Håkan Sundblad: Question Classification in Question Answering Systems, 2007.

No 1323 Magnus Lundqvist: Information Demand and Use: Improving Information Flow within Small-scale Business Contexts, 2007.

No 1329 Martin Magnusson: Deductive Planning and Composite Actions in Temporal Action Logic, 2007.

No 1331 Mikael Asplund: Restoring Consistency after Network Partitions, 2007.

No 1332 Martin Fransson: Towards Individualized Drug Dosage - General Methods and Case Studies, 2007.

No 1333 Karin Camara: A Visual Query Language Served by a Multi-sensor Environment, 2007.

No 1337 David Broman: Safety, Security, and Semantic Aspects of Equation-Based Object-Oriented Languages and Environments, 2007.

No 1339 Mikhail Chalabine: Invasive Interactive Parallelization, 2007.

No 1351 Susanna Nilsson: A Holistic Approach to Usability Evaluations of Mixed Reality Systems, 2008.

No 1353 Shanai Ardi: A Model and Implementation of a Security Plug-in for the Software Life Cycle, 2008.

No 1356 Erik Kuiper: Mobility and Routing in a Delay-tolerant Network of Unmanned Aerial Vehicles, 2008.

No 1359 Jana Rambusch: Situated Play, 2008.

No 1361 Martin Karresand: Completing the Picture - Fragments and Back Again, 2008.

No 1363 Per Nyblom: Dynamic Abstraction for Interleaved Task Planning and Execution, 2008.

No 1371 Fredrik Lantz: Terrain Object Recognition and Context Fusion for Decision Support, 2008.

No 1373 Martin Östlund: Assistance Plus: 3D-mediated Advice-giving on Pharmaceutical Products, 2008.

No 1381 Håkan Lundvall: Automatic Parallelization using Pipelining for Equation-Based Simulation Languages, 2008.

No 1386 Mirko Thorstensson: Using Observers for Model Based Data Collection in Distributed Tactical Operations, 2008.

No 1387 Bahlol Rahimi: Implementation of Health Information Systems, 2008.

No 1392 Maria Holmqvist: Word Alignment by Re-using Parallel Phrases, 2008.

No 1393 Mattias Eriksson: Integrated Software Pipelining, 2009.

No 1401 Annika Öhgren: Towards an Ontology Development Methodology for Small and Medium-sized Enterprises, 2009.

No 1410 Rickard Holsmark: Deadlock Free Routing in Mesh Networks on Chip with Regions, 2009.

No 1421 Sara Stymne: Compound Processing for Phrase-Based Statistical Machine Translation, 2009.

No 1427 Tommy Ellqvist: Supporting Scientific Collaboration through Workflows and Provenance, 2009.

No 1450 Fabian Segelström: Visualisations in Service Design, 2010.

No 1459 Min Bao: System Level Techniques for Temperature-Aware Energy Optimization, 2010.

No 1466 Mohammad Saifullah: Exploring Biologically Inspired Interactive Networks for Object Recognition, 2011 
Qiang Liu: Dealing with Missing Mappings and Structure in a Network of Ontologies, 2011.

Ruxandra Pop: Mapping Concurrent Applications to Multiprocessor Systems with Multithreaded Processors and Network on Chip-Based Interconnections, 2011.

No 1476

No 1481

No 1485

FiF-a 101

No 1490

No 1503

No 1504

No 1506

No 1507

No 1509

No 1510

No 1513

No 1523

No 1550

No 1558

No 1582

No 1586

No 1588

No 1589

No 1592

No 1606

No 1624
Per-Magnus Olsson: Positioning Algorithms for Surveillance Using Unmanned Aerial Vehicles, 2011.

Anna Vapen: Contributions to Web Authentication for Untrusted Computers, 2011.

Loove Broms: Sustainable Interactions: Studies in the Design of Energy Awareness Artefacts, 2011.

Johan Blomkvist: Conceptualising Prototypes in Service Design, 2011.

Håkan Warnquist: Computer-Assisted Troubleshooting for Efficient Off-board Diagnosis, 2011.

Jakob Rosén: Predictable Real-Time Applications on Multiprocessor Systems-on-Chip, 2011.

Usman Dastgeer: Skeleton Programming for Heterogeneous GPU-based Systems, 2011.

David Landén: Complex Task Allocation for Delegation: From Theory to Practice, 2011.

Kristian Stavåker: Contributions to Parallel Simulation of Equation-Based Models on

Graphics Processing Units, 2011.

Mariusz Wzorek: Selected Aspects of Navigation and Path Planning in Unmanned Aircraft Systems, 2011.

Piotr Rudol: Increasing Autonomy of Unmanned Aircraft Systems Through the Use of Imaging Sensors, 2011.

Anders Carstensen: The Evolution of the Connector View Concept: Enterprise Models for Interoperability

Solutions in the Extended Enterprise, 2011.

Jody Foo: Computational Terminology: Exploring Bilingual and Monolingual Term Extraction, 2012.

Anders Fröberg: Models and Tools for Distributed User Interface Development, 2012.

Dimitar Nikolov: Optimizing Fault Tolerance for Real-Time Systems, 2012.

Dennis Andersson: Mission Experience: How to Model and Capture it to Enable Vicarious Learning, 2013.

Massimiliano Raciti: Anomaly Detection and its Adaptation: Studies on Cyber-physical Systems, 2013.

Banafsheh Khademhosseinieh: Towards an Approach for Efficiency Evaluation of

Enterprise Modeling Methods, 2013.

Amy Rankin: Resilience in High Risk Work: Analysing Adaptive Performance, 2013.

Martin Sjölund: Tools for Understanding, Debugging, and Simulation Performance Improvement of EquationBased Models, 2013.

Karl Hammar: Towards an Ontology Design Pattern Quality Model, 2013.

Maria Vasilevskaya: Designing Security-enhanced Embedded Systems: Bridging Two Islands of Expertise, 2013. 